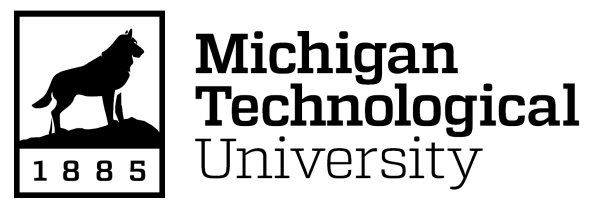

Michigan Technological University Digital Commons @ Michigan Tech

\title{
ESTIMATION AND PREDICTION OF THE HUMAN GAIT DYNAMICS FOR THE CONTROL OF AN ANKLE-FOOT PROSTHESIS
}

Guilherme Aramizo Ribeiro

Michigan Technological University, garamizo@mtu.edu

Copyright 2019 Guilherme Aramizo Ribeiro

\section{Recommended Citation}

Aramizo Ribeiro, Guilherme, "ESTIMATION AND PREDICTION OF THE HUMAN GAIT DYNAMICS FOR THE CONTROL OF AN ANKLE-FOOT PROSTHESIS", Open Access Dissertation, Michigan Technological University, 2019.

https://doi.org/10.37099/mtu.dc.etdr/863

Follow this and additional works at: https://digitalcommons.mtu.edu/etdr

Part of the Biomechanical Engineering Commons, Biomechanics and Biotransport Commons, Controls and Control Theory Commons, Robotics Commons, and the Signal Processing Commons 


\title{
ESTIMATION AND PREDICTION OF THE HUMAN GAIT DYNAMICS FOR THE CONTROL OF AN ANKLE-FOOT PROSTHESIS
}

\author{
By \\ Guilherme Aramizo Ribeiro

\begin{abstract}
A DISSERTATION
Submitted in partial fulfillment of the requirements for the degree of DOCTOR OF PHILOSOPHY

In Mechanical Engineering-Engineering Mechanics
\end{abstract}

MICHIGAN TECHNOLOGICAL UNIVERSITY

2019

(C) 2019 Guilherme Aramizo Ribeiro 
This dissertation has been approved in partial fulfillment of the requirements for the Degree of DOCTOR OF PHILOSOPHY in Mechanical Engineering-Engineering Mechanics.

\section{Department of Mechanical Engineering-Engineering Mechanics}

Dissertation Co-Advisor: $\quad$ Mo Rastgaar

Dissertation Co-Advisor: $\quad$ Ye Sun

Committee Member: Nina Mahmoudian

Committee Member: Timothy Havens

Department Chair: William Predebon 


\section{Table of Contents}

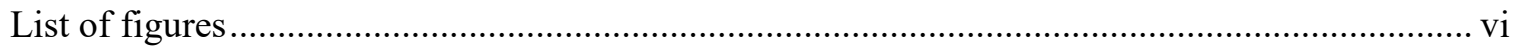

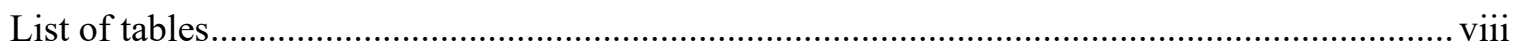

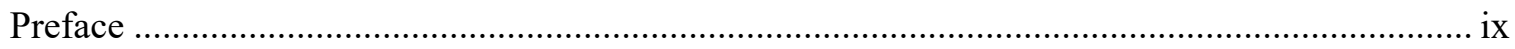

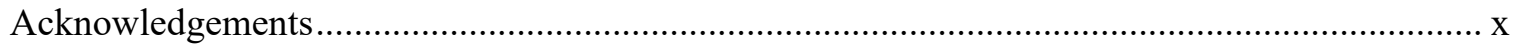

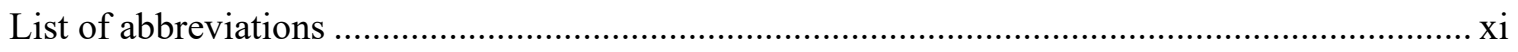

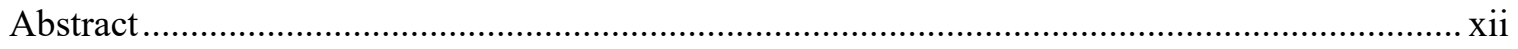

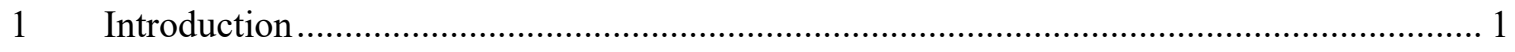

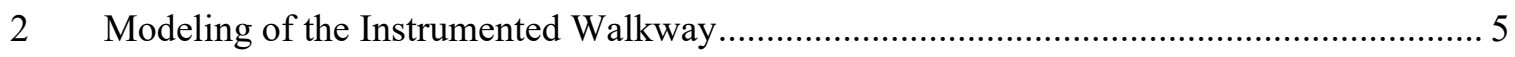

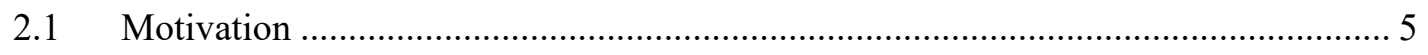

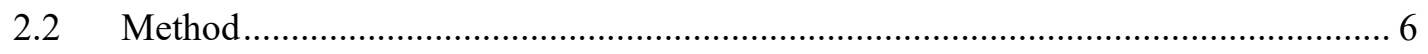

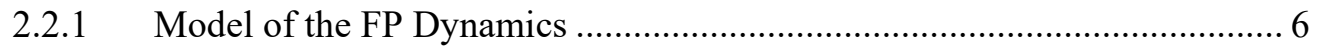

2.2.2 Experimental Estimation of the FP Inertial Parameters .............................. 8

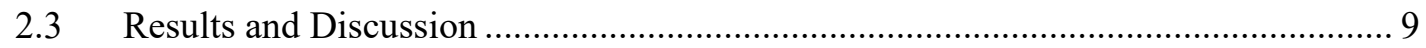

3 Mechanical Impedance of the Human Ankle over Levels of Muscle Co-Contraction ........ 11

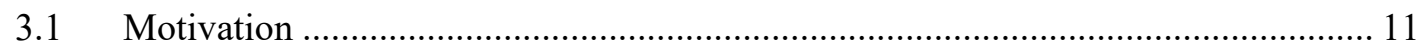

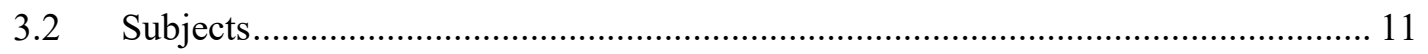

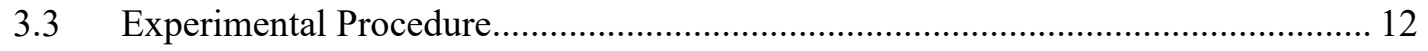

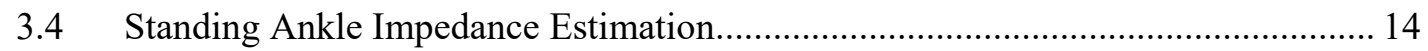

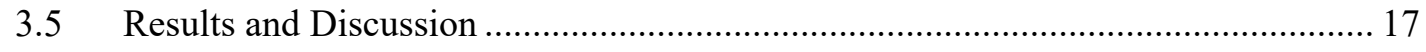

3.5.1 Evaluation of the Muscle Activity …...................................................... 17

3.5.2 Mechanical Impedance of the Ankle during Co-Contraction ..................... 18

3.5.3 Pair-wise Correlation Between Impedance Parameter and Muscle Activation 20 
4 Mechanical Impedance of the Human Ankle over Static Poses of the Gait Cycle

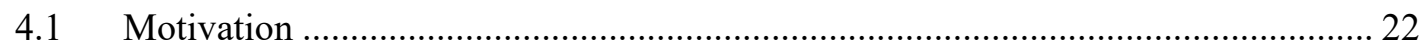

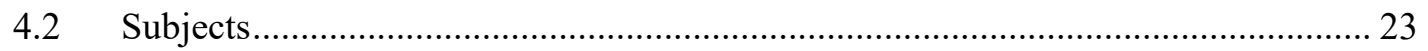

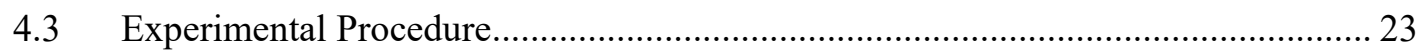

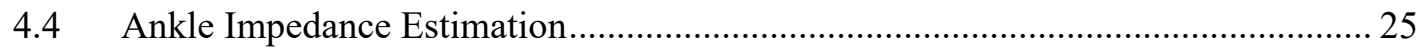

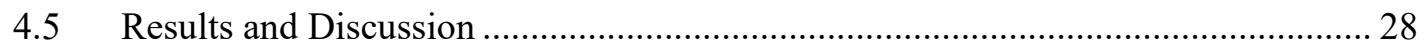

4.5.1 Range of Ankle Torque and Angle ....................................................... 28

4.5.2 Anisotropic Ankle Impedance ……….................................................... 29

5 Time-Varying Mechanical Impedance of the Human Ankle across the Stance Phase ........ 31

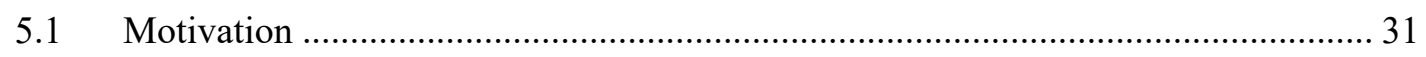

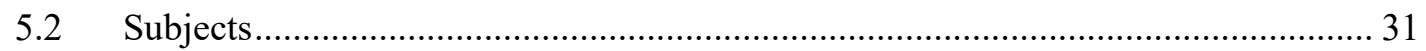

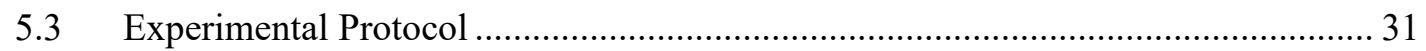

$5.4 \quad$ Identification of the Time-Varying Ankle Impedance........................................... 33

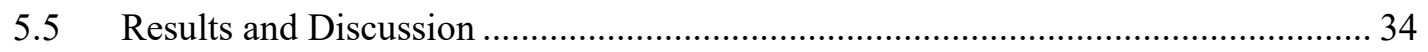

6 Design of a Vision Device to Assist Impedance Modulation of Ankle-Foot Prostheses ..... 38

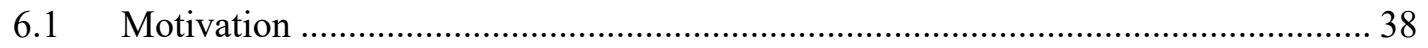

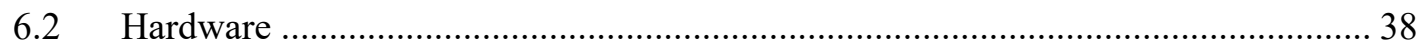

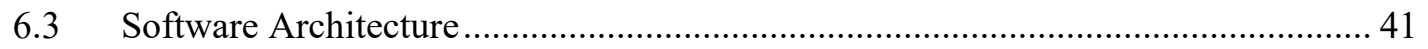

6.4 Extrinsic Calibration between the Camera and the IMU ....................................... 43

6.4.1 Calibration Procedure ………................................................................. 45

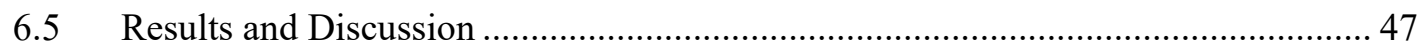

$7 \quad$ Heel Strike Prediction for a Prosthetic Device using Depth Vision..................................... 49

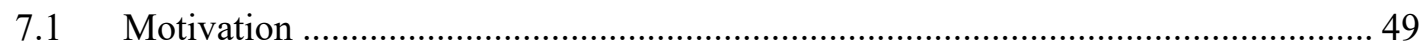

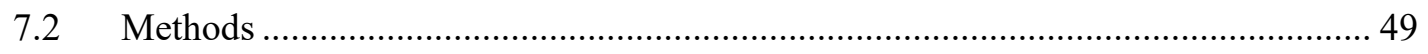

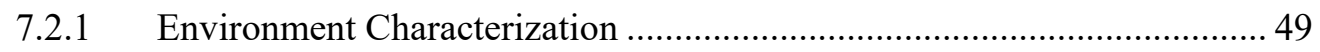


7.2.2 Gait Cycle Estimation

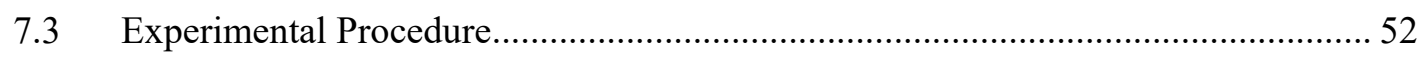

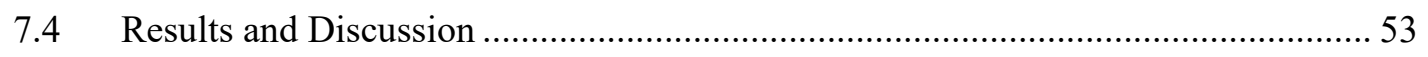

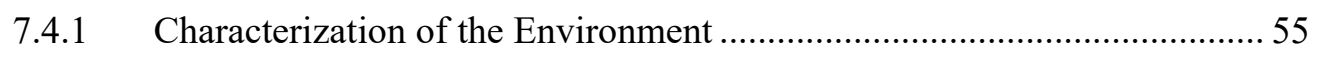

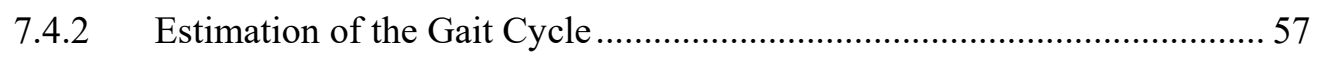

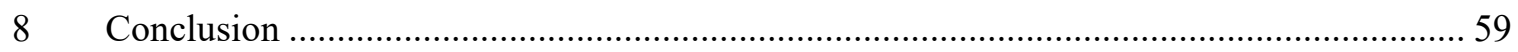

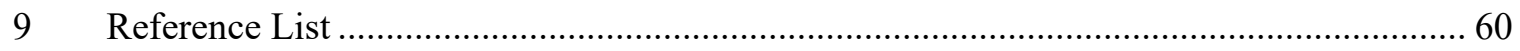




\section{List of figures}

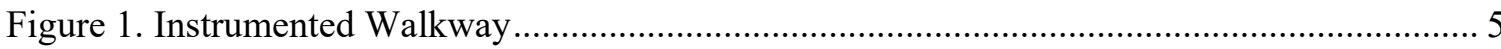

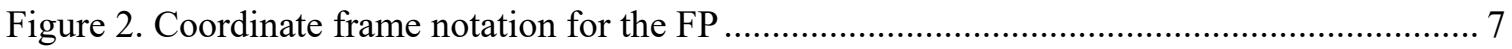

Figure 3. Subject in standing pose while the vibrating platform applied ground perturbations .... 12

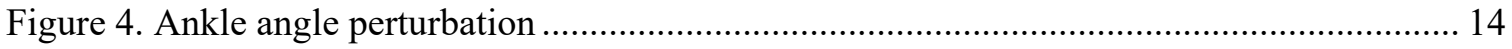

Figure 5. Normalized muscle activity for each of the co-contraction trials............................... 18

Figure 6. Mechanical ankle impedance in each of the co-contraction trials ................................ 19

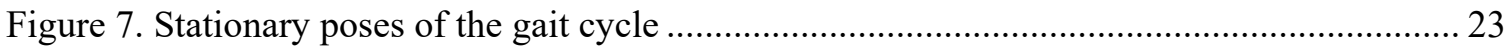

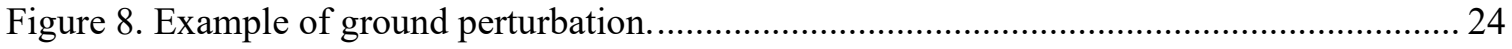

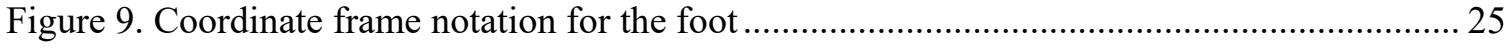

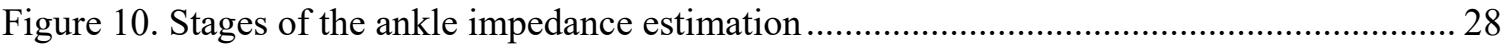

Figure 11. Mean angle and mean torque for the different stationary poses .................................2 29

Figure 12. Anisotropic ankle stiffness for four standing poses................................................. 30

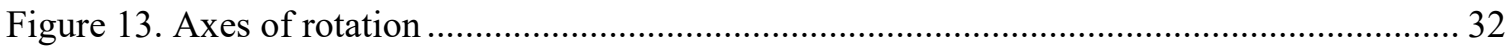

Figure 14. Average normalized time-varying ankle stiffness across the stance phase .................. 36

Figure 15. Average normalized time-varying ankle damping ............................................... 37

Figure 16. Components of the vision device used for gait assistance ........................................ 39

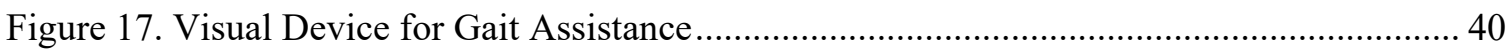

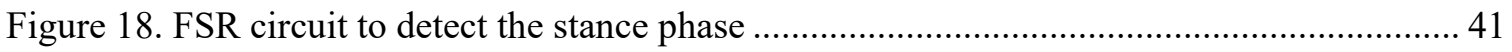

Figure 19. Graph of the processes executing on the VDGA system.......................................... 43 
Figure 20. Coordinate frame definitions of the VDGA

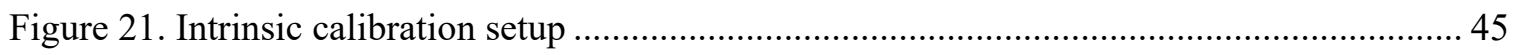

Figure 22. IMU orientation, linear acceleration, and angular velocity during the extrinsic calibration experiment..

Figure 23. Measurements from the VDGA during walking, showing the ground and a section of the subject's shoe

Figure 24. Point cloud processing pipeline 50

Figure 25. Walking course used for the evaluation of the VDGA. 53

Figure 26. Voltage on the FSR circuit across the gait cycle for the subject I 54

Figure 27. IMU measurements across the gait cycle for subject I 55

Figure 28. Normal vector estimates of the ground plane represented across the gait cycle .56

Figure 29. Histogram of the prediction error of the gait phase estimator .58 


\section{List of tables}

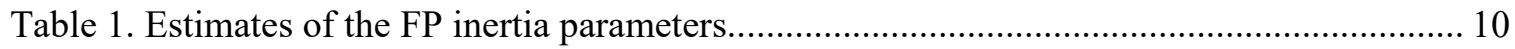

Table 2. Linear regression of impedance as a function of muscle contraction ............................. 20

Table 3. Evaluation of linearity between muscle contraction and impedance parameter .............. 21

Table 4. Environmental ground inclination for each experimental trial .................................... 57 


\section{Preface}

This dissertation is submitted in partial fulfillment of the requirements for a Degree of Doctor of Philosophy (Ph.D.) in Mechanical-Engineering. The work was conducted at the Mechanical Engineering-Engineering Mechanics PhD Program at Michigan Technological University under the supervision of Dr. Mo Rastgaar.

The research presented in this dissertation has been done by the author as well as in collaboration with others: Dr. Mo Rastgaar, Dr. Evandro Ficanha, Dr. Houman Dalalli, and Ms. Lauren Knop. All work was supported by the National Science Foundation, under grant numbers 1923760 and 1921046. 


\section{Acknowledgements}

I would like first to thank my academic advisor Dr. Mo Rastgaar, who gave me the opportunity and support to work on the Human Interactive Robotics Laboratory (HIRoLab). Since my first day at work, when I was only an undergraduate student in an exchange program, he has proposed engaging and impactful research challenges.

I am also grateful to Dr. Evandro Ficanha - at the time, a senior Ph.D. student working in HIRoLab - who I worked closely during my initial years of graduate studies. With him, I learned work ethics in research and how to enjoy the wilderness in the Upper Peninsula of Michigan.

I developed many more work relationships during my years in Michigan Technological University; At times, being the mentor, other times being the mentee. I am grateful for all the challenges we faced together because they helped me to develop not only my technical, but also my interpersonal, communication, and teamwork skills.

Finally, I would like to acknowledge my family, my friends in Brazil, and the friends I met in the United States for shaping the person I am today. Their love and support have allowed me to explore new endeavors and continuously reach for my dreams. Finalmente, eu gostaria de agradecer minha familia, meus amigos/as em Brasil, e os amigos/as eu conheci nos Estados Unidos por formar a pessoa que eu me tornei. Seu amor e suporte me possibilitou explorar novos caminhos e a sempre seguir meus sonhos. 


\section{List of abbreviations}

\begin{tabular}{|c|c|c|c|}
\hline ADL & Activities of Daily Living & MS & Midstance \\
\hline DP & Dorsiflexion-Plantarflexion & MS+ & Post Midstance \\
\hline IE & Inversion-Eversion & $\mathrm{TS}$ & Terminal Stance \\
\hline DOF & Degree(s) of freedom & $\mathrm{CoP}$ & Center of Pressure \\
\hline HS & Heel Strike & VDGA & Vision Device for Gait Assistance \\
\hline EMG & Electromyography & FSR & Force-Sensitive Resistor \\
\hline IMU & Inertial Measurement Unit & $\mathrm{ADC}$ & Analog-to-Digital Converter \\
\hline FP & Force Plate & ROS & Robotic Operational System \\
\hline CoM & Center of Mass & CW & Clock-Wise \\
\hline VAF & Variance Accounted For & $\mathrm{CCW}$ & Counter-Clock-Wise \\
\hline SNR & Signal to Noise Ratio & RANSAC & Random Sample Consensus \\
\hline MVC & Maximum Voluntary Contraction & & \\
\hline RMS & Root-Mean-Squared & & \\
\hline TA & Tibialis Anterior & & \\
\hline PL & Peroneus Longus & & \\
\hline SOL & Soleus & & \\
\hline GA & Gastrocnemius & & \\
\hline MAE & Mean Absolute Error & & \\
\hline ANOVA & Analysis Of Variance & & \\
\hline GAL & Gastrocnemius Lateral & & \\
\hline GAM & Gastrocnemius Medial & & \\
\hline FF & Foot Flat & & \\
\hline
\end{tabular}




\section{Abstract}

With the growing population of amputees, powered prostheses can be a solution to improve the quality of life for many people. Powered ankle-foot prostheses can be made to behave similar to the lost limb via controllers that emulate the mechanical impedance of the human ankle. Therefore, the understanding of human ankle dynamics is of major significance. First, this work reports the modulation of the mechanical impedance via two mechanisms: the co-contraction of the calf muscles and a change of mean ankle torque and angle. Then, the mechanical impedance of the ankle was determined, for the first time, as a multivariable and time-varying system. These findings reveal the importance of recognizing the state of the user during the gait when the user interacts with the environment. In addition to studying the ankle impedance, a wearable device was designed and evaluated to further the studies on robotic perception for ankle-foot prostheses. This device is capable of characterizing the ground environment and estimating the gait state using visual-inertial sensors. Finally, this study contributes to the field of ankle-foot prostheses by identifying the mechanical behavior of the human ankle and developing a platform to test perception algorithms for the control of robotic prostheses. 


\section{Introduction}

The incidence of amputations is a growing problem, currently affecting 2 million people in the United States, and is projected to affect approximately 3.6 million people in 2050 [1]. This steep increase is justified by the aging of the population around the world, and the increasing incidence of diabetes, which is the leading cause of amputations. Even more alarming is that this condition has a $50 \%$ mortality rate in the following 5 years after amputation due to vascular diseases [2]. Powered prostheses have the potential to help this community by recovering some of the mobility lost because of the amputation. When amputees are better assisted during activities of daily living (ADL), they have an opportunity to regain a healthier life.

Currently, there are many transtibial powered prostheses available commercially [3]-[5]. $\mathrm{BiOM}$ can decrease the metabolic cost of the gait by applying an ankle torque during toe-off. It includes an embedded controller that adapts to the user cadence and ground level [4]. Another prosthesis that adapts to the terrain is the Ossur's Proprio Foot, which can dorsiflex the foot during the swing to provide foot clearance [5]. Endolite's Elan uses hydraulics to adjust ankle resistance [3], mimicking the ankle's behavior. Orson et al. included an active transversal DOF to reduce shear stress and rotation of the residual limb in the socket [6] since it was shown to cause abrasion and skin problems [7]. However, a major concern for the control of these prostheses is how to modulate the compliance of the prosthesis as it interacts mechanically with the ground.

This compliance can be measured as mechanical impedance. The mechanical impedance defines how much reactive torque the ankle generates when an external disturbance changes the ankle angle. Some prostheses are capable of modulating the ankle impedance [8]-[15] and experimental devices were developed to test the accuracy of this modulation [16], [17]. A good approach to control these prostheses would be to modulate the same ankle mechanical impedance of the amputee prior to the amputation. Thus, studying the mechanical properties of unpaired subjects might provide useful insights into the design of new control strategies for powered prostheses.

Early developments on ankle impedance estimation go back to the 1980s with Weiss, Kearney, and Hunter [18]-[20]. They estimated a $2^{\text {nd }}$ order impedance of the ankle of subjects lying in the supine position, and noticed that the ankle position can cause an increase in stiffness and 
damping components up to a factor of five and eight, respectively [18]. Furthermore, a related finding showed that the ankle impedance was modulated by an increase of the ankle torque. This study found increases in the stiffness by about $10 \mathrm{Nm} / \mathrm{rad}$ for every $\mathrm{Nm}$ torque unit [19]. In other words, the ankle impedance in sagittal plane and at a steady-state changed according to the ankle position and active torque; both of these being unique to different activities of daily living. Recently, it has been reported that the ankle impedance might be more dynamically complex than a $2^{\text {nd }}$ order system. Therani suggests that the ankle impedance should be modeled by a $3^{\text {rd }}$ order system rather than the $2^{\text {nd }}$ order spring-mass-damper, matching a Hill-type muscle model [21].

Studies of the ankle impedance in the sagittal plane extended to functional activities, such as straight walking [22] and turning [23]. The early stance phase was studied by Rouse et al. using a vibrating platform, called Perturberator, that applies torque perturbations to the ankle from the ground. They found a linearly increasing stiffness from $20 \%$ to $70 \%$ of the gait cycle, ranging between 105-455 Nm/rad; and an increasing damping at later stages, ranging between 0-2.8 $\mathrm{Nms} / \mathrm{rad}$ (impedance de-normalized by subject mass of $70 \mathrm{~kg}$ ) [24]. The swing phase was studied by Lee and Hogan [25] using an exoskeleton, Anklebot. They verified that both the stiffness and damping form a concave-up parabola with a minimum value around mid-swing. The average sagittal stiffness and damping was $40 \mathrm{Nm} / \mathrm{rad}$ and $1.2 \mathrm{Nms} / \mathrm{rad}$, respectively. These results suggest that the decrease in stiffness and damping around mid-swing might facilitate toe clearance, and the increase in stiffness and damping after mid-swing might prepare the ankle for a ground impact during the heel-strike. Finally, the terminal stance was studied by Shorter et al. [26]. However, the estimated ankle stiffness during walking was estimated to be different of the earlier findings that studied the steady-state impedance. This indicates that there are more unknown factors modulating the ankle impedance besides ankle torque and position.

Even though the dorsi-plantar (DP) motion is the primary focus of most studies, there are substantial ankle motions in all other anatomical planes during walking, such as in inversioneversion (IE) [27]-[30]. Thus, understanding the characteristics of the ankle impedance in different planes of motion is essential. Lee and Rastgaar studied the anisotropic stiffness with active muscles, finding that the quasi-stiffness took the form of a "peanut shape" [31], [32]. They used Anklebot to estimate the quasi-static stiffness of the ankle along 24 directions of rotation and for different combinations of muscle engagement. The stiffness was more substantial around the sagittal plane and smaller around the frontal plane, ranging between 10-45 Nm/rad and 5-15 Nm/rad, 
respectively. The multi-variate study about the impedance was extended to a dynamic test, with stochastic analyses and larger muscle activation ranges. The DP and IE stiffness increased with the lower-extremity muscle activation. The growth in stiffness was approximately the same for all muscle activation trials, with a ratio of 3.8 .

The anisotropic ankle impedance during walking was estimated with a two Degrees of Freedom (DOF) vibrating platform for the first time [23]. Step torque perturbations were applied along different moments of the gait cycle, at 16 different axes of rotation. A $2^{\text {nd }}$ order model was estimated via least square, resulting in the inertia, damping, and stiffness parameters of the ankle. During heel-strike (HS), the modulated stiffness around IE rotations is higher than around DP, possibly as a mechanism to increase the stability against ankle roll. After HS, the modulated stiffness has a major axis along PI (plantar-inversion) and DE (dorsi-eversion), with a certain symmetry about the subtalar joint. Both stiffness and damping reach their highest values between $23-38 \%$ of the gait cycle. These results suggest a time-varying, multi-variable analysis of the ankle impedance is essential for the control of prostheses.

For powered prostheses to modulate a time-varying impedance or simply to follow a timebased trajectory, such as the ankle torques and angles across the gait cycle, they need to know the state of the user, such as the gait phase [33], [34]. In addition, many gait maneuvers are of substantial importance during ADL [35]. Amputees compensate for the lack of ankle motion control in the sagittal plane using other joints in the body, such as the hip [36]. There has been much work on classifying the user's intent and features of the environment for prosthesis control. Another approach to detect a user's intention with the prosthesis is via Electromyography (EMG) of the lower leg muscles, with many studies relating the impedance with the muscle activity [37][44].

An emerging approach is to use exteroceptive sensors, which can measure the environment features directly. This can assist prostheses in adapting the behavior based on the ground terrain, slope [45], and flatness [46]. A ranging laser and an inertial measurement unit (IMU) attached on the hip has been used to estimate the terrain type and locomotion mode of able-bodied and amputee subjects, with a high accuracy of 98\% [47]. Krauz used a Microsoft Kinect (which creates an image that corresponds each pixel to the spatial distance between the camera and the point) to characterize nearby stairs [48]. Another unique benefit of exteroceptive sensors is that they are less dependent 
on user's characteristics, as demonstrated by Massalin [49]. They were able to predict ambulation modes using a minimal number of training subjects. Ranging sensors are also able to estimate the state of the sound-leg to assist the coordination between prostheses and users [50].

In this dissertation, the mechanical impedance of the human ankle was studied and related to multiple other factors. In Chapter 2, the instrumental apparatus used to quantify multi-directional ankle dynamics is presented and characterized. After the experimental apparatus was evaluated, the ankle impedance was estimated and related to the muscle activity of the lower leg (Chapter 3), to operating points of angle and torque (Chapter 4), and during walking (Chapter 5). Given the influence of the gait state and environment to the control of prosthesis, a vision device was designed and evaluated for gait assistance applications (Chapter 6), and preliminary results are presented in Chapter 7. 


\section{Modeling of the Instrumented Walkway}

\subsection{Motivation}

The Instrumented Walkway is an experimental apparatus designed to study the dynamics of the human ankle as they change across gait maneuvers. As seen in Figure 1, it consists of a two degrees of freedom (DOF) vibrating platform, a force plate (Kistler 9260AA3), a motion capture camera system (8 Optitrack 17W cameras), and a set of wireless surface EMG sensors (Delsys Trigno Wireless System). The force plate (FP), the motion capture camera system, and the EMG sensors are used to measure the external torque acting on the ankle, the ankle angles, and the activation of the calf muscles, respectively, while the vibrating platform applies ground perturbations on the ankle. The vibrating platform is driven by two linear motors via Bowden cables and a pulley system. The cable setup allows the platform to rotate around any of the horizontal axes of rotation, resulting in a combination of pitch and roll motions, but not a yaw motion. The construction of the Instrument Walkway is fully reported in Ficanha et al. [12].

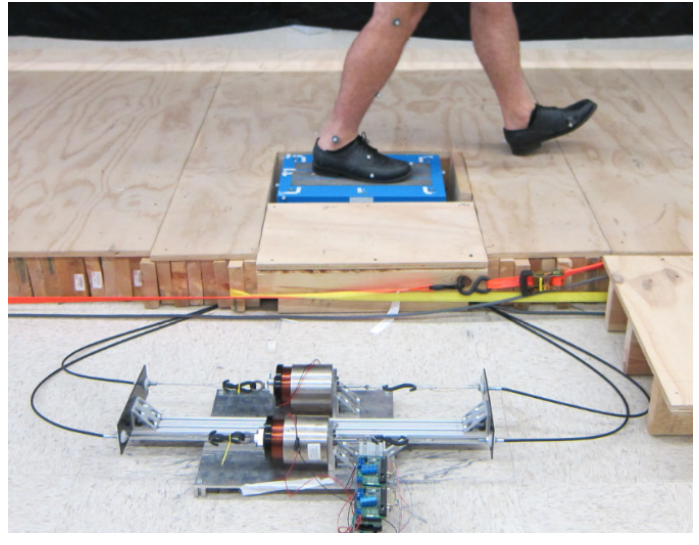

(a)

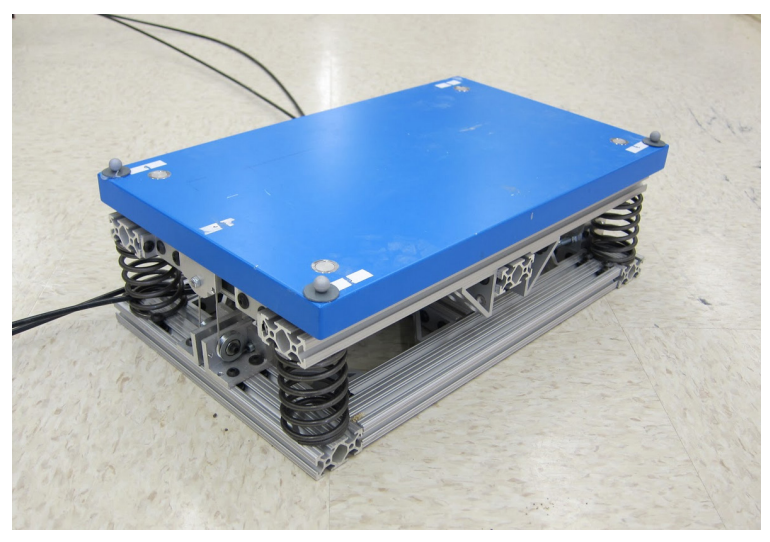

(b)

Figure 1. Instrumented Walkway. (a) During a walking experiment and (b) close view of the vibrating mechanism.

The Instrumented Walkway was designed to identify the dynamics of the human ankle without being affected by the dynamics of the actuators, except for limiting the frequency bandwidth of the input signal. This was accomplished by assembling a FP on top of the vibrating platform capable of measuring the interface forces and torques to the human subjects. By considering these forces and torques as the new input to the system, rather than the voltage 
commands to the actuators, all the actuator dynamics can be disregarded during the identification of the human ankle.

However, because the FP measures all the dynamics occurring above the force sensors, the inertial dynamics of the FP are still accounted for in the measurements. The inertial dynamics are substantial considering the large mass of the FP compared to the average human foot. Therefore, the force and torque measurements of the FP must be compensated. This chapter describes the method to compensate for the dynamics of the Instrumented Walkway, specifically the inertial parameters of the FP, and is further used in Chapters 3 and 4.

\subsection{Method}

The inertial dynamics of the FP can be compensated from the human trials in the following procedure:

1) Model the forces and torques measured by the FP as a function of the FP kinematics (position, velocity, and acceleration) and FP inertial parameters (moment of inertia, product of inertia, mass, and center of mass);

2) Record a "calibration" experiment prior to the human trials in which an unloaded vibrating platform is actuated (without human subjects);

3) Estimate the FP inertial parameters using the calibration measurements and the mathematical model; and

4) For the human trials, predict and compensate the forces and torques using the previously estimated model and FP kinematics of the current trial.

\subsubsection{Model of the FP Dynamics}

A coordinate frame is defined in the center of the top surface of the FP, on point $P$ (Figure 2). The measured force and torque, $F_{P}$ and $T_{P}$, respectively, are defined in this coordinate frame.

Assuming that nothing is in contact with the top surface of the FP and that the vibrating platform is moving the FP via an interface force and torque, the motion of the system can be modeled by the law of conservation of linear and angular momentum around point $\mathrm{P}$ : 


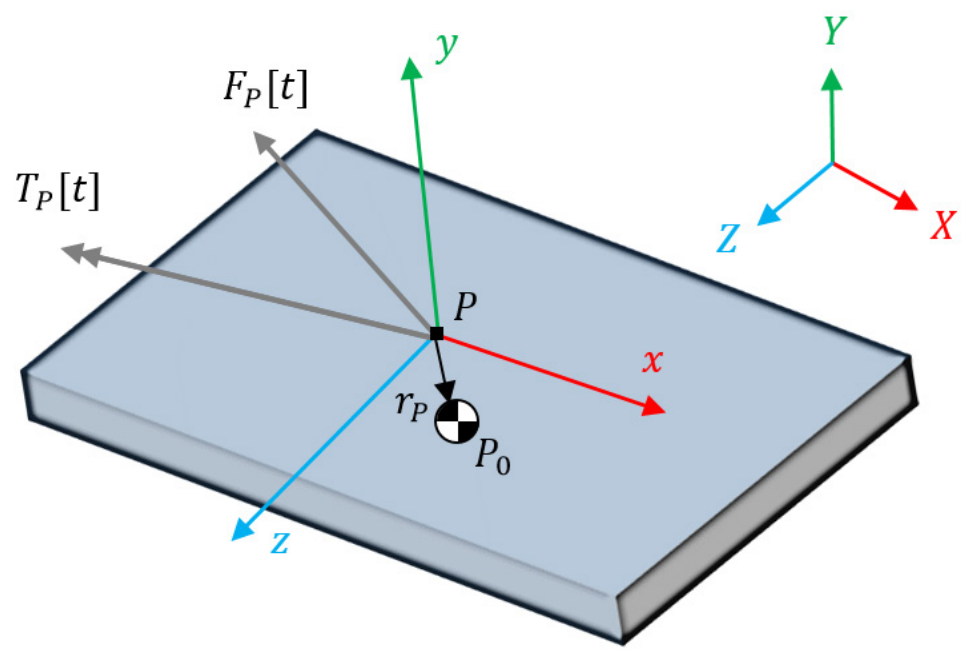

Figure 2. Coordinate frame notation for the FP. Origin of the body lies in the center of the top surface, with x pointing towards the long body dimension and y towards the normal to the top surface. The measured force and torque, $\mathrm{T}_{\mathrm{P}}[\mathrm{t}]$ and $\mathrm{F}_{\mathrm{P}}[\mathrm{t}]$, act on the origin of the body

When the platform is vibrated during an unloaded scenario, only the weight and the contact forces and torques (measured) are external actors on the body. In this case, the equation of the linear motion can be derived from the linear momentum, $p$, as

$$
\begin{gathered}
\sum F=\frac{d p}{d t} \\
F_{P}+m_{P} g=\frac{d\left(m_{P} \dot{S}_{P_{0}}\right)}{d t} \\
F_{P}=m_{P}\left(\ddot{s}_{P_{0}}-g\right)
\end{gathered}
$$

Similarly, the angular motion can be derived from the angular momentum about the moving point $\mathrm{P}, L_{P}$

$$
\begin{gathered}
\sum_{P} T=\frac{d L_{P}}{d t} \\
T_{P}+r_{P} \times m_{P} g=\frac{d\left(I_{P} \omega_{P}+r_{P} \times m_{P} \dot{S}_{P_{0}}\right)}{d t}
\end{gathered}
$$




$$
\begin{gathered}
=I_{P} \dot{\omega}_{P}+\omega_{P} \times\left(I_{P} \omega_{P}\right)+r_{P} \times m_{P} \ddot{S}_{P_{0}}+\left(\omega_{P} \times r_{P}\right) \times m_{P} \dot{S}_{P_{0}} \\
T_{P}=I_{P} \dot{\omega}_{P}+\omega_{P} \times\left(I_{P} \omega_{P}\right)+r_{P} \times m_{P}\left(\ddot{S}_{P_{0}}-g\right)+\left(\omega_{P} \times r_{P}\right) \times m_{P} \dot{S}_{P}
\end{gathered}
$$

for $I_{P}=\left[\begin{array}{ccc}I_{P}^{x x} & I_{P}^{x y} & I_{P}^{x z} \\ I_{P}^{x y} & I_{P}^{y y} & I_{P}^{y z} \\ I_{P}^{x z} & I_{P}^{y z} & I_{P}^{z z}\end{array}\right]$,

where $m_{P}, r_{P},\left\{I_{P}^{x x}, I_{P}^{y y}, I_{P}^{z z}\right\}$, and $\left\{I_{P}^{y z}, I_{P}^{x z}, I_{P}^{x y}\right\}$ are the inertial parameters of the FP: the mass, the center of mass $(\mathrm{CoM})$ relative to the FP origin, the moment of inertia, and product of inertia about the CoM; In addition,

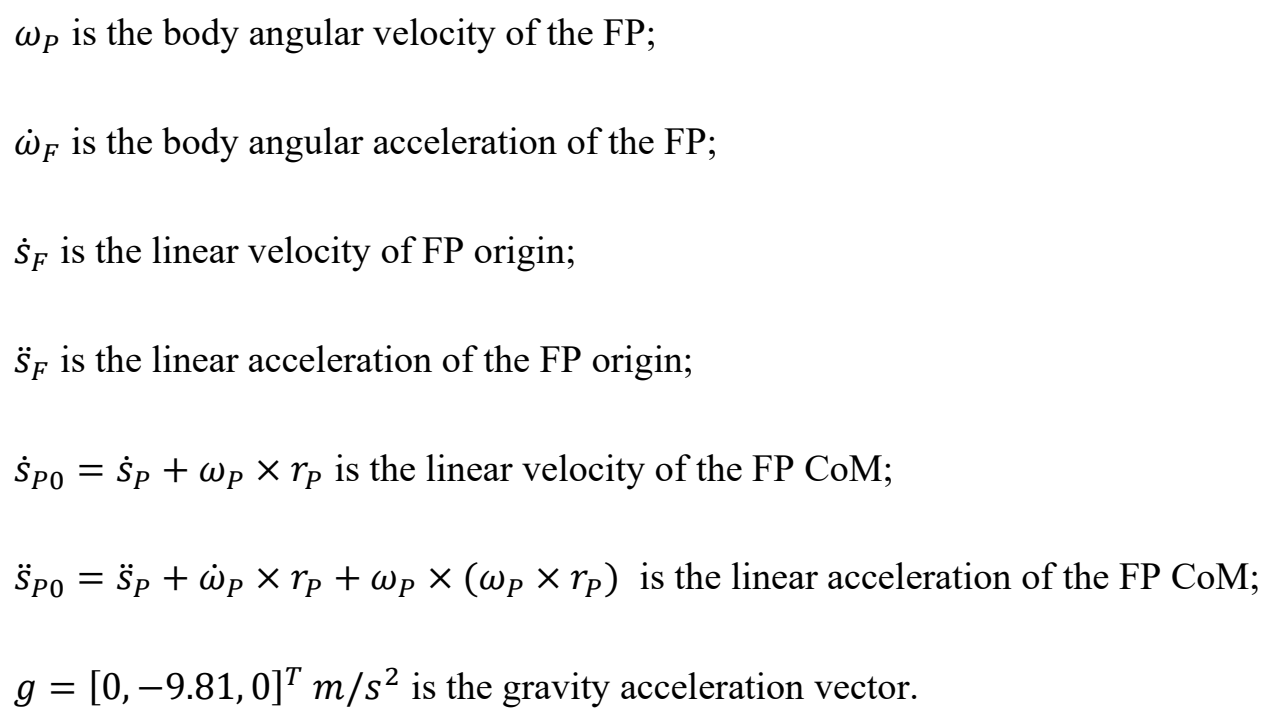

\subsubsection{Experimental Estimation of the FP Inertial Parameters}

The unknown parameters from Eq. (2.3) and (2.) are estimated from a calibration experiment and later used to compensate the ground reaction forces and torques from the human experiments. In this experiment, the unloaded vibrating platform was actuated with a random signal $(30 \mathrm{~Hz}$ update rate) for 30 seconds. This estimation can be solved as a problem of non-linear optimization of the form

$$
\theta^{*} \triangleq \underset{\theta}{\operatorname{argmin}} \sum_{t} \varepsilon_{\theta}[t]^{T} \varepsilon_{\theta}[t]
$$


where

$$
\begin{gathered}
\varepsilon_{\theta}[t] \triangleq\left[\begin{array}{c}
I_{P} \dot{\omega}_{P}[t]+\omega_{P}[t] \times I_{P} \omega_{P}[t]+r_{P} \times m_{P}\left(\ddot{s}_{P_{0}}[t]-g\right)-\dot{s}_{P}[t] \times m_{P} \dot{s}_{P_{0}}[t]+T_{\text {bias }}-T_{P}[t] \\
m_{P}\left(\ddot{s}_{P_{0}}[t]-g\right)+F_{\text {bias }}-F_{P}[t]
\end{array}\right] \\
\theta \triangleq\left[\begin{array}{lllllllll}
I_{P}^{x x} & I_{P}^{y y} & I_{P}^{z z} & I_{P}^{y z} & I_{P}^{x z} & I_{P}^{x y} & r_{P}^{T} & T_{\text {bias }}^{T} & F_{\text {bias }}^{T}
\end{array}\right]^{T}
\end{gathered}
$$

are the cost function (with implicit dependence to $\theta$ ) derived from the residual of Eq. (2.3) and (2.6), and the vector of unknown parameters, respectively. The operator $*[t]$ represents a measurement from time $t$. $T_{\text {bias }}$ and $F_{\text {bias }}$ were added to Eq. (2.) to represent a constant torque and force, respectively, due to sensor zeroing and bias noise. The bias noise increases steadily over time, but can be approximated as constant for the short duration of an average human experiment. This bias noise is compensated with a sensor zeroing by subtracting a constant value from the force and torque measurement, turning these measurements to zero (at the moment of the zeroing operation). However, because sensor zeroing also subtracts the force and torque components due to the weight, the parameters $T_{\text {bias }}$ and $F_{\text {bias }}$ must be added to correct the cost function in Eq. (2.8).

Signal Processing. The time derivatives were numerically computed via Savitzky-Golay filter [51] ( $5^{\text {th }}$ order polynomial in a 15 -samples window) and the optimization solved with MATLAB's fmincon function (interior-point algorithm [52]).

\subsection{Results and Discussion}

The FP inertia parameters were estimated as $[35.0 \pm 1.9,131.7 \pm 18.8,119.6 \pm 2.5]^{T} \mathrm{~g} \cdot \mathrm{m}^{2}$, $[0.1 \pm 1.2,1,9 \pm 0.4,0.4 \pm 1.2]^{T} \mathrm{~g} \cdot \mathrm{m}^{2}$, and $[4.8 \pm 1.3,-6.9 \pm 2.9,2.3 \pm 1.3]^{T} \mathrm{~mm}$ for the moment of inertia, the product of inertia, and the center of mass vector, respectively. The variance accounted for (VAF) of the torque and force reconstruction (Eq. 2.3 and 2.6) were $[95.7 \pm 1.3,85.1 \pm 12.9,98.0 \pm 0.8]^{T}$ and $[95.3 \pm 2.7,85.4 \pm 4.3,97.5 \pm 1.3]^{T}$, respectively. The mass parameter (mass of the components above the force sensor) was reported by the manufacturer to be $4.64 \mathrm{~kg}$, and was fixed during the estimation. 
Table 1. Estimates of the FP inertia parameters. Columns of each parameter represent X, Y, and Z components, respectively.

\begin{tabular}{r|ccc|ccc|ccc} 
& \multicolumn{3}{|c}{$\boldsymbol{J}_{\boldsymbol{P}}\left[\boldsymbol{g} . \mathbf{m}^{\mathbf{2}}\right]$} & \multicolumn{3}{c|}{$\boldsymbol{P}_{\boldsymbol{P}}\left[\boldsymbol{g} . \boldsymbol{m}^{2}\right]$} & \multicolumn{3}{c}{$\boldsymbol{r}_{\boldsymbol{P}}[\mathbf{m m}]$} \\
\hline Ref & 34.8 & 131.2 & 97.0 & 0.0 & 0.0 & 0.0 & 0.0 & -14.4 & 0.0 \\
Min & 31.5 & 108.2 & 115.4 & -2.2 & 1.2 & -1.4 & 2.2 & -10.0 & -0.6 \\
Mean & 35.0 & 131.7 & 119.6 & 0.1 & 1.9 & 0.4 & 4.8 & -6.9 & 2.3 \\
Max & 37.0 & 149.6 & 122.7 & 1.3 & 2.4 & 2.0 & 6.7 & -1.8 & 3.7 \\
Std & 1.9 & 18.8 & 2.5 & 1.2 & 0.4 & 1.2 & 1.3 & 2.9 & 1.3
\end{tabular}

The FP moment of inertia and product of inertia were similar to an ideal box of equivalent size and mass. This ideal box would have a moment of inertia of $[34.8,131.2,97.0]^{T} \mathrm{~g} \cdot \mathrm{m}^{2}$ and $0.0 \mathrm{~g} . \mathrm{m}^{2}$ product of inertia. The small estimated product of inertia (consistent with the symmetric shape of the body) indicates that the mass inside the FP case is well distributed. The large variance of $18.8 \mathrm{~g} \cdot \mathrm{m}^{2}$ for the $J_{P}^{y}$ estimate is expected because the vibrating platform cannot move in this axis of rotation; thus, the signal to noise ratio (SNR) around this axis is small. Similarly, the estimated center of mass vector, $r_{P}$, has a high standard deviation of $[1.3,2.9,1.3]^{T} \mathrm{~mm}$, indicating a high uncertainty. Possibly the confidence of $r_{P}$ could be improved if the FP underwent an unconstrained motion, rather than a constrained motion (pivoting about the universal joint).

Finally, for this application, the VAF of the reconstructed (predicted) forces and torques are more relevant than the confidence of the inertial parameters. This implies that, to predict and compensate for the effects of the FP dynamics on the human experiments, the inertial forces and torques must be calculated accurately (high VAF), but it is not necessary that the parameters that contribute little to the measured forces and torques (such as the product of inertia and CoM) are estimated with high confidence. 


\section{Mechanical Impedance of the Human Ankle over Levels of Muscle Co-Contraction}

\subsection{Motivation}

The human ankle is a complex joint whose motion involves multiple bones, tendons, and muscles. For example, ankle rotations in the DP and IE directions require the synergistic work of twelve and five of the lower-leg muscles, respectively. Faced with such complexities, new developments in machine learning have been exploring the prediction of the intended ankle motion of amputees using muscle contraction commands [53]-[56]. A direct application of motion prediction is the control of powered prosthesis via muscles of the residual limb. One example of such a controller developed by Wang et al. [57], who used the EMG signals to proportionally drive the DP angle of a prosthesis. Another implementation predicted the desired angle during walking activities using a nonlinear auto-regressive model from muscle signals inside the prosthesis socket [58].

Muscles contribute to the overall ankle motion, and with the activation of antagonistic muscles, it can also affect its mechanical impedance. Studies in this field have demonstrated that muscle contraction contributes to a significant increase in the ankle stiffness in the DP direction [19]. Additionally, the impedance was shown to be different depending on the axis and direction of rotation, and changed for various combinations of muscles being activated [31], [59]. In this work, the study of the ankle impedance with active muscles was extended to subjects in the standing pose to further the understanding of the ankle dynamics.

\subsection{Subjects}

Twelve able-bodied male subjects with no self-reported history of biomechanical or neuromuscular disorders were recruited (age of $27.9 \pm 3.5$ years, weight of $92.3 \pm 27.6 \mathrm{~kg}$, and height of $180.2 \pm$ $6.7 \mathrm{~cm}$ ). All participants gave written informed consent to participate in this study, which was approved by the Michigan Technological University Institutional Review Board. 


\subsection{Experimental Procedure}

The experiment consisted of ten 70-seconds trials in which random perturbations were applied to the right feet of the standing subjects (Figure 3). For the duration of each trial, the subjects stood with feet facing one out of two directions (forward (a) or to the side (b), Figure 3) and co-contracted their lower-leg muscles at a fixed activation level out of 5 levels $(0 \%, 10 \%, 20 \%, 30 \%$, and $40 \%$ of the maximum voluntary contraction (MVC)). The subjects had the assistance of a real-time chart of their muscle activity while holding the muscle contraction level. The maximum selected cocontraction level was $40 \%$ MVC because the average subject could not hold this level of activity for more than 70 seconds. The initial 10 seconds of each trial were discarded from further analyses, to reduce the transient effect.

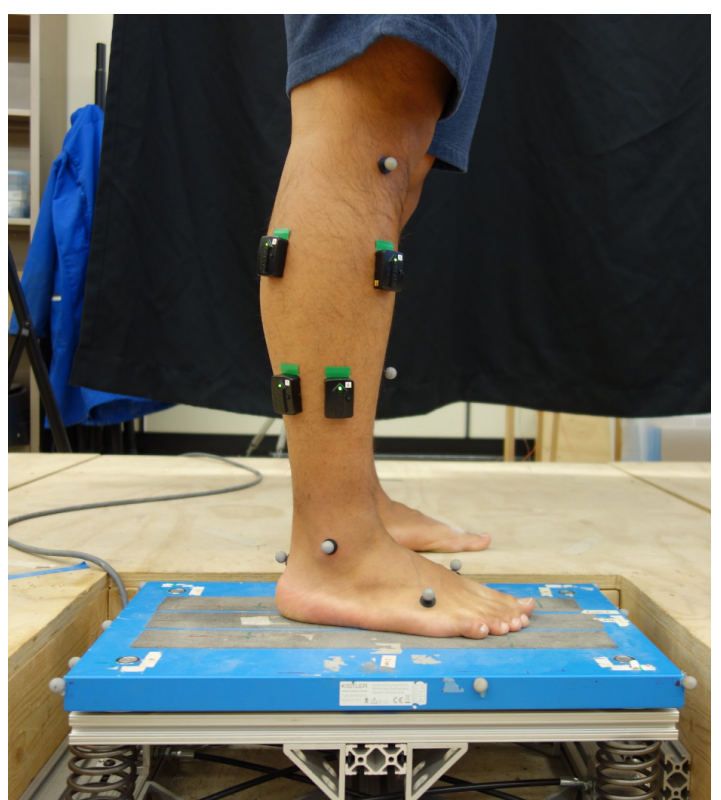

(a)

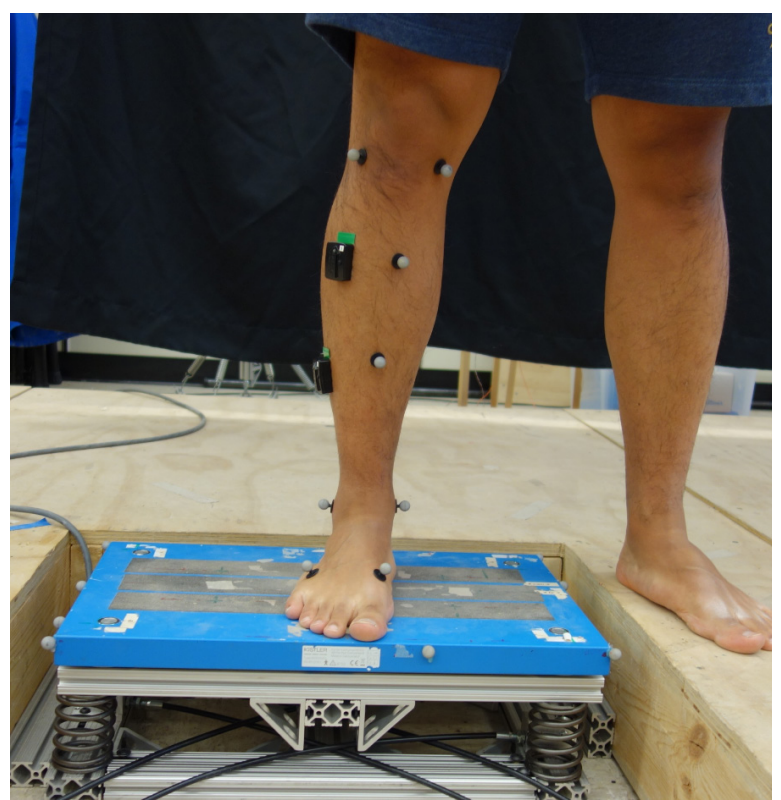

(b)

Figure 3. Subject in standing pose while the vibrating platform applied ground perturbations.

EMG sensors were placed on the TA, PL, SOL, and GA EMG muscles.

To determine the MVC of a subject, prior to the experiment, the subjects contracted their lower-leg at their maximum levels in pulses of 1-second for 5 to 10 times. The MVC was defined as the maximum recorded voltage of the TA muscle and used as a reference for the subsequent trials. In these subsequent trials, the subjects tried to co-contract all muscles of the lower-leg while looking at a real-time graph of the $20 \mathrm{~ms}$ rolling root-mean-square (RMS) of the TA muscle as visual feedback. The target MVC was overlaid on the same graph. Finally, the trials were performed 
in random order and with rest intervals of more than one minute to reduce the effect of muscle fatigue on the aggregated results.

Measurements were taken from the foot, shank, and force plate kinematics, external torques and forces perturbations, all sampled at $350 \mathrm{~Hz}$. Additionally, the muscle activity of the tibialis anterior (TA), peroneus longus (PL), soleus (SOL), and gastrocnemius (GA) muscles were measured and sampled at $2000 \mathrm{~Hz}$. These muscles were selected based on their contribution to ankle stabilization and rotation [15]. The EMG signals were low-passed filtered by the Delsys filtering software to reduce motion artifact. A detailed description of the instrumental apparatus is described in Chapter 2.

The random perturbation used in this experiment was random in both the magnitude and directions of rotation, changing its value every 0.03 seconds. The magnitude of the signal was in the form of pseudo-random binary sequences and the direction was uniformly distributed across all combinations of dorsiflexion, plantarflexion, inversion, and eversion rotations. Note that the dynamics of the actuators acted as a filter to this signal, limiting the bandwidth of the random input. A sample of the signal is shown in Figure 4 in the time and frequency domain.

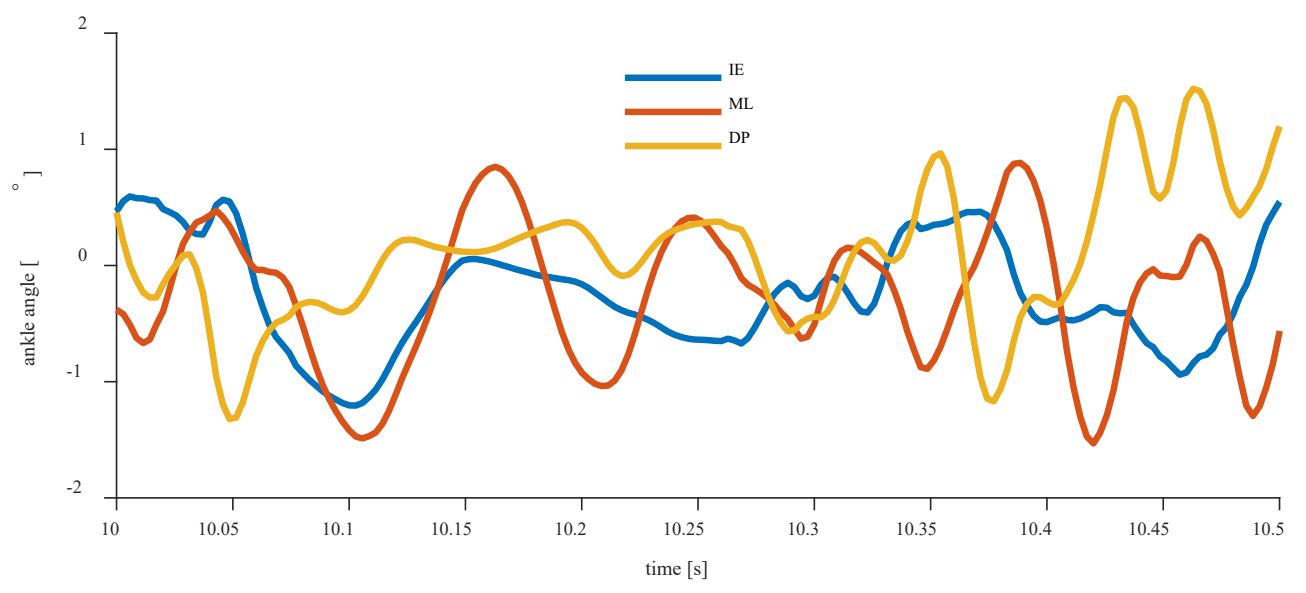

(a) 


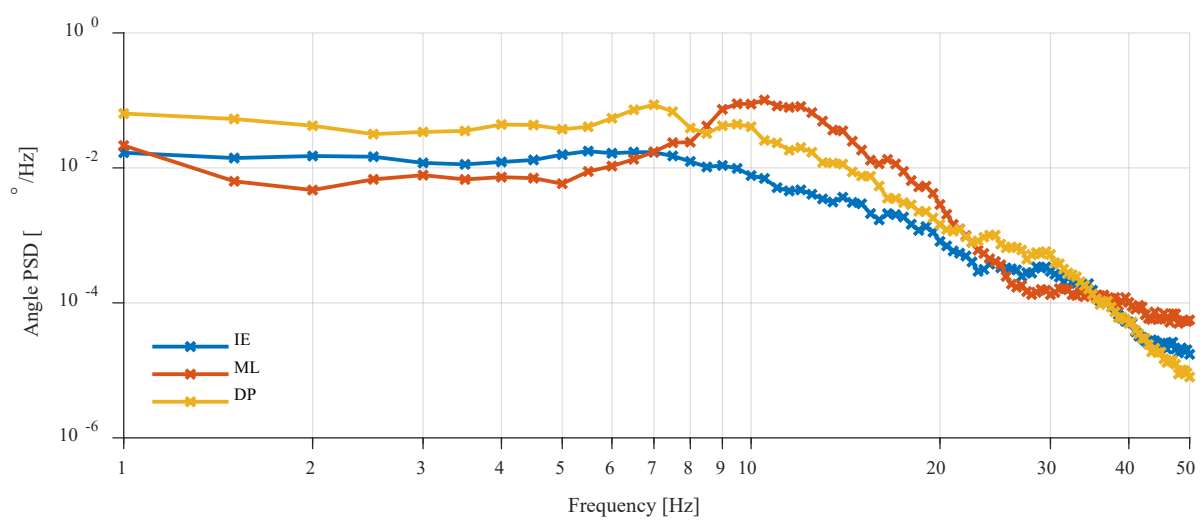

(b)

Figure 4. Ankle angle perturbation. Represented in the (a) time domain and (b) frequency domain.

\subsection{Standing Ankle Impedance Estimation}

The multivariable mechanical impedance of the ankle was estimated using the differential equations of motion of the lower leg that included the ankle impedance coefficients and the inertia of the foot. This method approximates the foot as a rigid body

$$
\begin{gathered}
\sum_{F} T=\frac{d L_{F}}{d t} \\
T_{F}+r_{F} \times m_{F} g+T_{Z}(\theta, \dot{\theta})=\frac{d\left(I_{P} \omega_{P}+r_{P} \times m_{P} \dot{s}_{P_{0}}\right)}{d t} \\
=I_{F} \dot{\omega}_{F}+\omega_{F} \times\left(I_{F} \omega_{F}\right)+r_{F} \times m_{F} \ddot{s}_{F_{0}}+\left(\omega_{F} \times r_{F}\right) \times m_{F} \dot{s}_{F_{0}} \\
T_{F}=I_{F} \dot{\omega}_{F}+\omega_{F} \times\left(I_{F} \omega_{F}\right)+r_{F} \times m_{F}\left(\ddot{s}_{F_{0}}-g\right)+\left(\omega_{F} \times r_{F}\right) \times m_{F} \dot{s}_{F}+T_{Z}(\theta, \dot{\theta})
\end{gathered}
$$

where

$m_{F}$ is the foot mass;

$r_{F}$ is the center of mass relative to the foot origin; 
$I_{F}=\left[\begin{array}{lll}I_{F}^{x x} & I_{F}^{x y} & I_{F}^{x z} \\ I_{F}^{x y} & I_{F}^{y y} & I_{F}^{y z} \\ I_{F}^{x z} & I_{F}^{y z} & I_{F}^{z z}\end{array}\right]$ is the inertia matrix of the foot relative to the CoM;

$\left\{I_{F}^{x x}, I_{F}^{y y}, I_{F}^{z z}\right\}$ are the moments of inertia of the foot, relative to the CoM;

$\left\{I_{F}^{y z}, I_{F}^{x z}, I_{F}^{x y}\right\}$ are the products of inertia of the foot, relative to the CoM;

$\omega_{F}$ is the body angular velocity of the foot;

$\dot{\omega}_{F}$ is the body angular acceleration of the foot;

$\dot{S}_{F}$ is the linear velocity of foot origin;

$\ddot{S}_{F}$ is the linear acceleration of the foot origin;

$\dot{s}_{F 0}=\dot{s}_{F}+\omega_{F} \times r_{F}$ is the linear velocity of the foot CoM and,

$\ddot{s}_{F 0}=\ddot{s}_{F}+\dot{\omega}_{F} \times r_{F}+\omega_{F} \times\left(\omega_{F} \times r_{F}\right)$ is the linear acceleration of the foot CoM.

$T_{F}$ is the external torque acting on the ankle, compensated by the FP inertia, calculated as

$$
T_{F}=T_{P}-T_{i P}+\left(s_{P}-s_{F}\right) \times\left(F_{P}-F_{i P}\right)
$$

where $T_{P}, F_{P}, T_{i P}, F_{i P}, s_{P}$, and $s_{F}$ are the external ground torque, the external ground force, inertial FP torque (2.3), inertia FP force (2.6), FP position, and foot position, respectively.

The variable $T_{Z}(\theta, \dot{\theta})$ is the torque due to the mechanical ankle impedance. The ankle was modeled as a gimbal joint with springs and viscous dampers on each of the three consecutive rotating axes in the order XYZ: first a rotation in the shank's X (IE) axis, then on the new Y (ML) axis, and finally on the foot's Z (DP) axis. These rotations are represented as the ankle rotation vector, $\theta \in \mathbb{R}^{3}$, and generate torque in the form

$$
T_{Z}(\theta, \dot{\theta})=J_{X Y Z}^{-1}(\theta)\left(\left[\begin{array}{ccc}
K_{I E} & 0 & 0 \\
0 & K_{M L} & 0 \\
0 & 0 & K_{D P}
\end{array}\right] \theta+\left[\begin{array}{ccc}
B_{I E} & 0 & 0 \\
0 & B_{M L} & 0 \\
0 & 0 & B_{D P}
\end{array}\right] \dot{\theta}\right)
$$




$$
J_{X Y Z}^{-1}(\theta)=\frac{1}{\cos \theta_{Y}}\left[\begin{array}{ccc}
\cos \theta_{Z} & -\sin \theta_{Z} & 0 \\
\cos \theta_{Y} \sin \theta_{Z} & \cos \theta_{2} \cos \theta_{Z} & 0 \\
-\sin \theta_{Y} \cos \theta_{Z} & \sin \theta_{2} \sin \theta_{Z} & \cos \theta_{Y}
\end{array}\right]
$$

where $J_{X Y Z}^{-1}(\theta)$ is the mapping between the body angular velocity and the Euler (XYZ) angle rates [60], which, conversely, maps a torque from Euler coordinates to foot coordinates.

The time-derivatives were calculated with a Sarvitzky-Golay filter [51] with 11-samples window and a 5th order polynomial. In addition, the same filter was used to smooth all the other kinematic signals. This filter approximates the samples of a signal within a moving window as a polynomial and calculates derivatives with good noise rejection.

The best-fit estimates for the unknown parameters were calculated with a non-linear optimization method, Sequential Quadratic Programming [52], by substituting the measurements and computed derivatives into Eq. 1, and reducing the mean-square-error of the equation. In addition, to account for sensor biases and time-varying impedance different values of $T_{\text {bias }}, F_{\text {bias }}$, $\mathrm{K}$ and $\mathrm{B}$ were estimated in small sample windows. Therefore, the vector of unknown parameters is

$$
x \equiv\left[I_{F}^{x x}, I_{F}^{y y}, I_{F}^{z z}, I_{F}^{y z}, I_{F}^{x z}, I_{F}^{x y}, m_{F}, r_{F}^{T}, z_{0 \%}^{T} z_{10 \%}^{T}, z_{20 \%}^{T}, z_{30 \%}^{T}, z_{40 \%}^{T}\right]^{T}
$$

for

$$
z_{i}^{T} \equiv\left[T_{b i a s, i}^{T}, F_{b i a s, i}^{T}, K_{i}^{T}, B_{i}^{T}\right]^{T}
$$

where $z_{i}$ is the set of biases and impedances of trial $i$.

Considering the impedance and bias might change within a trial, each trial was split in 40 time-windows of 2 -seconds of duration (25\% overlap) and used to estimate an independent solution, x. Therefore, each subject had 40 estimates of foot inertia, and 400 estimates of ankle impedance.

Once the unknown parameters are estimated, the model can be evaluated measuring the mean absolute error (MAE) of the torque as 


$$
M A E_{x}=\frac{1}{N} \sum_{i=1}^{N}\left\|\boldsymbol{T}_{F}^{[i]}(\boldsymbol{x})-\boldsymbol{T}_{\text {ref }}^{[i]}\right\|_{1}
$$

where $\boldsymbol{T}_{F}^{[i]}(\boldsymbol{x})$ and $\boldsymbol{T}_{\text {ref }}^{[i]}$ are the estimated and measured ground reaction torques around the ankle ( $T_{F}$ from Equation 3.3), for the $i^{\text {th }}$ of $\mathrm{N}$ samples in a trial. The normalized mean square error $\left(\mathrm{R}^{2}\right)$ is another measure of model confidence, but it is normalized by the overall variance of the reference signal.

\subsection{Results and Discussion}

\subsubsection{Evaluation of the Muscle Activity}

A summary of the normalized EMG signals during each co-contraction trial is presented in Figure 5. The RMS of the EMG signal across the trial is calculated for each subject and grouped with the respective co-contraction trial in a boxplot. Therefore, the presented percentile statistics are calculated across the subject population (does not account for variation across the trial). The EMG was z-score normalized within each subject to allow comparisons between subjects.

The median EMG activity increased linearly as intended on the experimental design, especially from the TA muscles. The TA was the only muscle directly supervised during the experiment, so it is expected to follow the linear pattern. However, the PL, GA, and specially SOL showed more deviations from a linear increase, suggesting that some subjects could not increase the muscle activity proportionally (at least without real-time feedback, as provided for TA). 


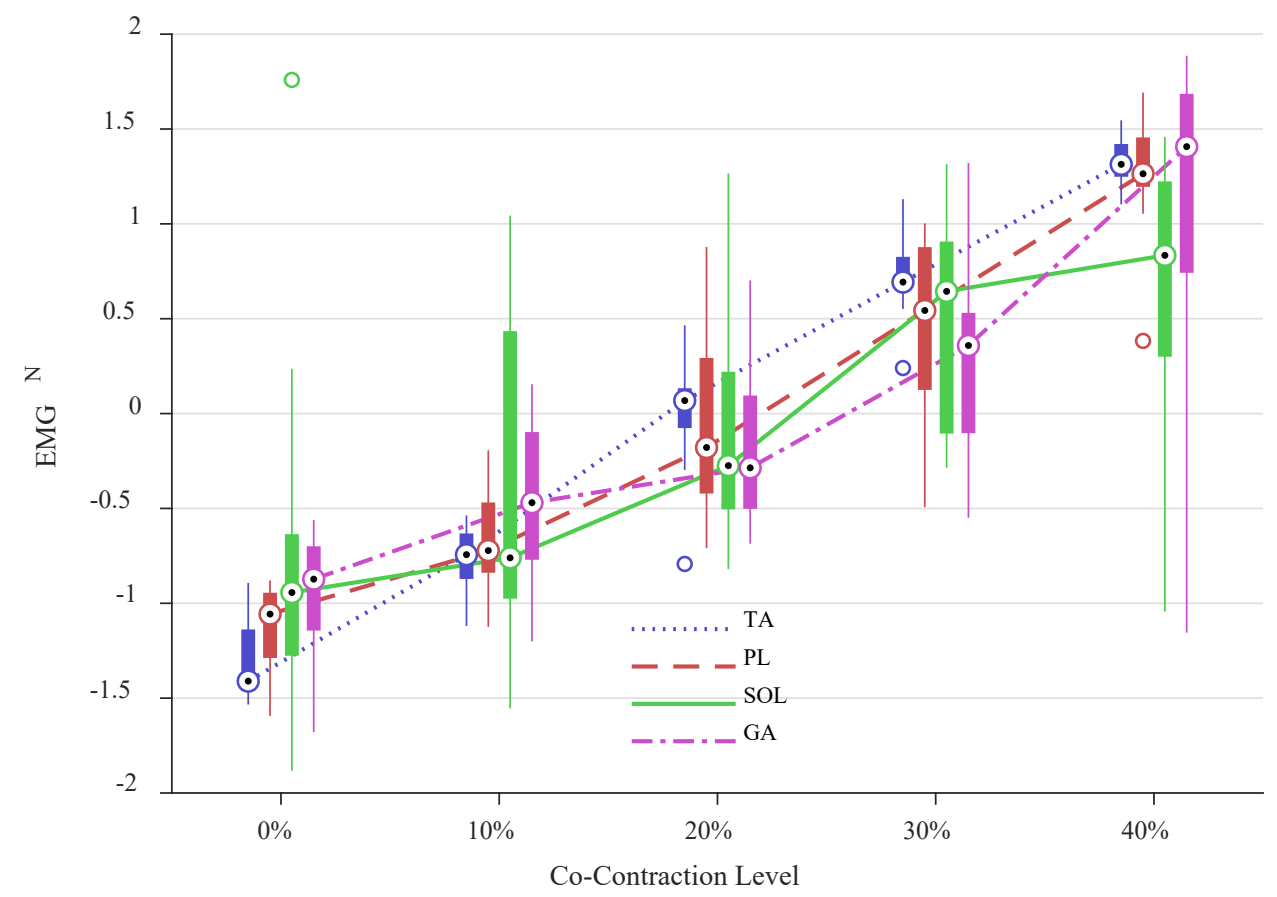

Figure 5. Normalized muscle activity for each of the co-contraction trials. The percentiles of the box plots are computed across the RMS of the EMG signal for each subject.

\subsubsection{Mechanical Impedance of the Ankle during Co-Contraction}

The external torque was predicted using the estimated impedance parameters and resulted in a MAE torque of $2.1 \pm 0.3 \mathrm{Nm}$ (average and the standard deviation were calculated across all subjects and trials). The model $\mathrm{R}^{2}$ values were above 0.8 and 0.7 for most subjects along DP and IE anatomical axes, respectively, and above 0.5 for all subjects, in any co-contraction level (Figure 6). The DP $\mathrm{R}^{2}$ decreased with increasing co-contraction levels, while IE $\mathrm{R}^{2}$ peaked at the $20 \%$ MVC trial and showed the lowest overall fitness at $0 \%$ MVC. 


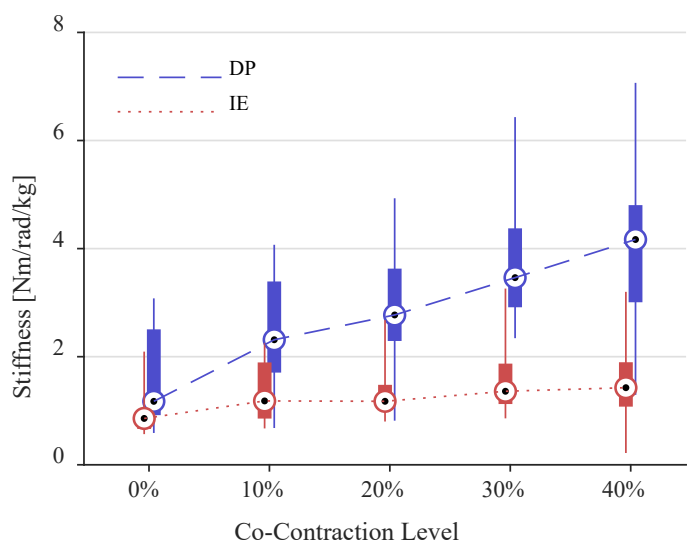

(a)

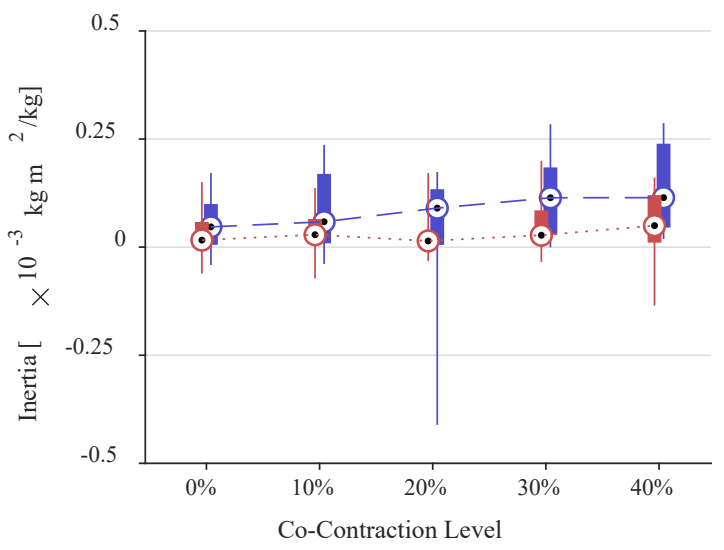

(c)

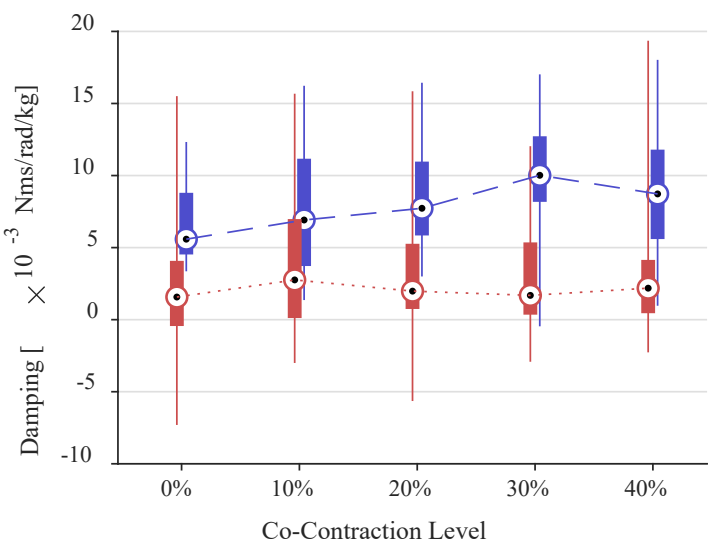

(b)

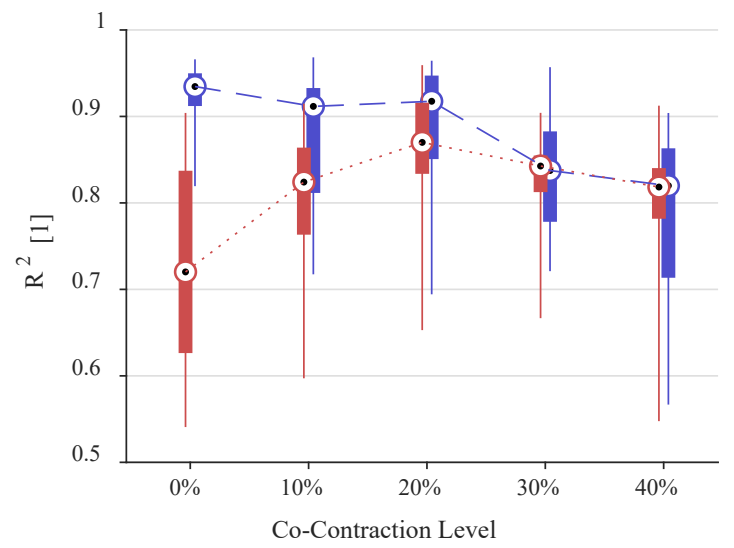

(d)

Figure 6. Mechanical ankle impedance in each of the co-contraction trials. The impedance was parameterized with a (a) stiffness, (b) damping, and (c) inertia coefficients, and evaluated with the (d) $R^{2}$ of the response ankle torque.

The DP stiffness component of the impedance significantly increased with the cocontraction level, while the median of the IE stiffness, DP and IE damping, and DP and IE inertia remained relatively constant (Table 2). All stiffness and damping parameters were significantly greater than zero and had similar values to the non-loaded ankle conditions [20], [59]. The DP stiffness range was four times greater than the IE stiffness range, while DP damping was higher than IE damping in all conditions, consistent with previous results [59]. The insensitivity of the IE stiffness to muscle contraction and co-contraction was also observed in previous studies [31]. 
Table 2. Linear regression of impedance as a function of muscle contraction. * represents coefficients significantly different than zero (t-test, significance $\mathrm{p}<0.05$ )

\begin{tabular}{|c|c|c|c|c|}
\cline { 2 - 5 } \multicolumn{1}{c|}{} & $\boldsymbol{K}_{\boldsymbol{D} \boldsymbol{P}}$ & $\boldsymbol{K}_{\boldsymbol{I E}}$ & $\boldsymbol{B}_{\boldsymbol{D} \boldsymbol{P}}$ & $\boldsymbol{B}_{\boldsymbol{I E}}$ \\
\cline { 2 - 5 } \multicolumn{1}{c|}{} & \multicolumn{2}{c|}{$\mathrm{Nm} / \mathrm{rad}$} & \multicolumn{2}{c|}{$\mathrm{Nms} / \mathrm{rad}$} \\
\hline Passive Impedance (Intercept) & $154.7^{*}$ & $98.5^{*}$ & $0.67^{*}$ & $0.27^{*}$ \\
\hline Impedance change (per \% MVC) & $5.2^{*}$ & 1.3 & 0.01 & 0.0 \\
\hline Line Fit $\boldsymbol{R}^{\mathbf{2}}$ & 0.42 & 0.1 & 0.0 & 0.0 \\
\hline
\end{tabular}

\subsubsection{Pair-wise Correlation Between Impedance Parameter and Muscle Activation}

Possibly the large impedance variance amongst subjects and the not-significant impedance change (per \% MVC), as shown in Figure 6, could have been caused by subjects failing to hold muscle activity levels consistently (Figure 5). Therefore, it might be worthwhile to search for a muscleimpedance relationship for each subject separately. To that end, a pair-wise relationship between one muscle to one impedance parameter was tested with a linear model. For each subject, the EMG RMS was calculated along the 2-second time-window. A single representative point of EMG and impedance was calculated as the median across all the time-windows, resulting in five points in an EMG-impedance graph (that is, five points in a Cartesian plane, where the $\mathrm{x}$-axis is EMG RMS, and the $y$-axis is impedance parameter). The medians were used in this analysis to address the variation of the EMG and impedance within each trial.

The linearity of these points was evaluated with an Analysis of Variance (ANOVA, $\mathrm{p} \leq$ 0.05 ) for linear models. All the combinations of muscles and impedances were analyzed, totaling sixteen linear models per subject. Two subjects were removed from this analysis because they presented irregular EMG measurements. The percentage of subjects presenting linear correlation is shown in Table 3. 
Table 3. Evaluation of linearity between muscle contraction and impedance parameter.

Percentage of subjects who presented linear correlation between medians of EMG RMS and average impedance.

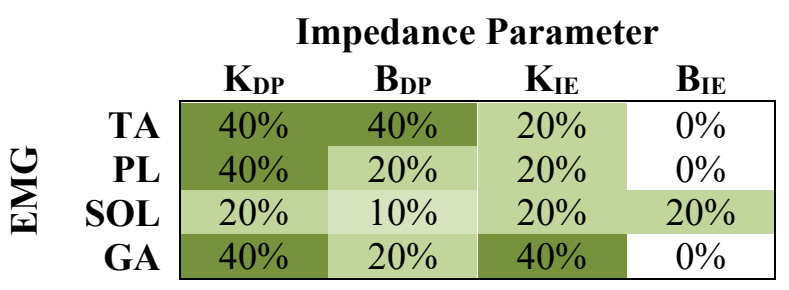

The DP stiffness parameter showed the most frequency of linear correlations with other muscles (up to $40 \%$ for some muscles), while IE damping showed the least ( $0 \%$ for most muscles). Interestingly, DP damping and IE stiffness showed a high frequency of correlation to TA and GA muscles' EMG, respectively, even though they have low overall change per MVC (Table 2). This suggests that muscle activity may be a good predictor for the mechanical impedance of the ankle; however, a predictor model would require a more complex structure than a linear model. 


\section{Mechanical Impedance of the Human Ankle over Static Poses of the Gait Cycle}

\subsection{Motivation}

The characterization of the mechanical impedance has been a rich field of research for many decades due to the complexity of the ankle joint complex. For example, the ankle has been shown to respond differently around different mean ankle angles and torques. Weiss et al. identified the DP mechanical impedance of the ankle as a $2^{\text {nd }}$ order model whose parameters are dependent on the mean ankle angle and torque [18], [19]. They verified that when holding the same ankle position, stiffness increases linearly with the torque. The reflexive properties of the impedance also changed, arising from complex neural mechanisms [61]. In addition, the damping coefficient increased by a factor of eight at extreme ankle angles away from the neutral angle. Not only does the magnitude of the rotation affect the impedance, but also the direction, as reported by Hyunglae et al. [31], [59]. The polar plot of the stiffness (versus the ankle axis of rotation) took the form of a peanut shape with the longer axis pointing close to the DP axis. In a static experiment, the stiffness for dorsiflexion was higher than in plantar-flexion [31], showing that ankle rotations in the same axis, but in different directions, also have different dynamic responses.

These studies have been extended to the ankle impedance in the standing position, which accounts for the complexity of body sway and forces loading on the ankle. Considerable body sway was found to increase the value of the stiffness, possibly as an active neural control response to stabilize the body pose [62]. In addition, the non-neural response, referred to as the intrinsic stiffness, increases when the body sways forward [63]. However, the angle deviations arising from body sway are minimal compared to the full range of the ankle angle during ADLs, such as walking. In this chapter, the mechanical impedance of the ankle was estimated while subjects stood in varying angles and torques operating points, with similar values found during walking. 


\subsection{Subjects}

Fifteen subjects with no self-reported neuromuscular or biomechanical disorders participated in this experiment (ages of $28.0 \pm 4.4$, mass of $79.0 \pm 11.1 \mathrm{~kg}$, and height of $178.0 \pm 7.7 \mathrm{~cm}$ ). The subjects gave written consent to participate in the experiment, which was approved by the Michigan Tech Institutional Review Board.

\subsection{Experimental Procedure}

The Instrumented Walkway (Chapter 2) was used in this experiment. In addition, EMG sensors (Delsys Trigno wireless ${ }^{\mathrm{TM}}, 2000 \mathrm{~Hz}$ sampling rate) were used to measure the lower extremity muscle activity. The sensors were placed on 5 muscles; including the TA, the PL, the SOL, the gastrocnemius lateral (GAL), and the gastrocnemius medial (GAM). These muscles were selected based on their antagonistic properties and their contribution to motion in the DP and IE axes [64].

This study analyzed fours stationary poses: Foot Flat (FF), Midstance (MS), Post Midstance (MS+), and Terminal Stance (TS), as shown in Figure 7. Each of these poses has a different combination of ankle angle and foot center of pressure $(\mathrm{CoP})$ to emulate the state of the body in various stages of the gait cycle. However, different from walking, the subjects remained stationary at each one of the poses throughout a full trial. The experiment was divided into three rounds, each with one trial per pose; these trials were executed in random order and lasted 35 seconds each, with a 1-minute rest interval.

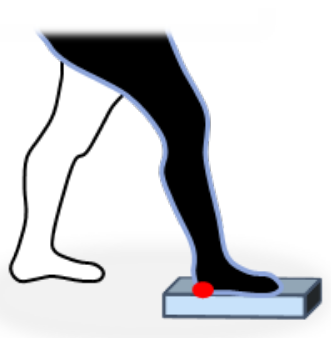

$\mathrm{FF}$

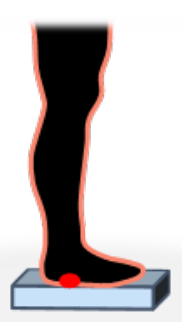

MS

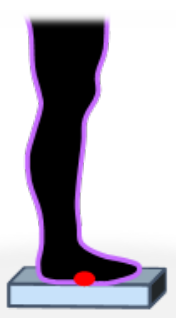

MS+

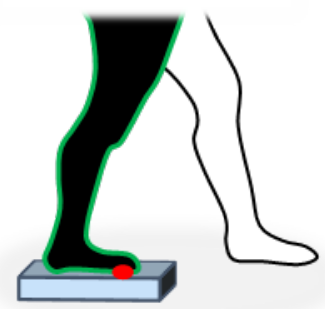

TS

Figure 7. Stationary poses of the gait cycle. The poses emulate moments of the stance phase of the walk. The CoP increases along the anterior-posterior direction for the FF, MS, MS+, and TS poses, from left to right. The red dot indicates the CoP location. 
To have consistency between repeated trials, the placement of the feet, the CoP's anteriorposterior position (Equation 4.1), and the weight distribution were supervised. The right foot was placed on the force plate inside a drawn outline of the foot, while the left foot was behind (FF), aligned (MS, MS+), or in front (TS) of the right foot. For the FF and TS poses, the stance length (anterior-posterior distance between the feet) was defined as $40 \%$ of the subjects' height. Lastly, the subjects relied on real-time measurements on a monitor screen to maintain the CoP of the perturbed foot around a desired target location and hold equal weight distribution between both legs. The target CoP locations were $30.6 \%, 40.5 \%, 53.0 \%$, and $63.6 \%$ of the foot length, from the heel, for the poses FF, MS, MS+, and TS, respectively.

$$
C O P_{x}=\left(T_{P}^{Z} / F_{P}^{y}-d_{P . h e e l}\right) / L_{f o o t}
$$

where $d_{P . h e e l}$ and $L_{\text {foot }}$ are the foot length and distance from heel to the center of FP. $T_{P}$ and $F_{P}$ are the torque and force measurements by the force plate.

The ground perturbations were in the form of pulse trains of random rotating axis $\left(0-360^{\circ}\right)$, period (0.03-0.2 s), and duration (0.9-1.1 s); and between consecutive pulse trains, the vibrating platform was inactive for a random pause time (0.9-1.1 s), as shown in Figure 8. The use of random durations and pause periods was intended to decrease reflex responses and predictive muscle contraction by the subjects.

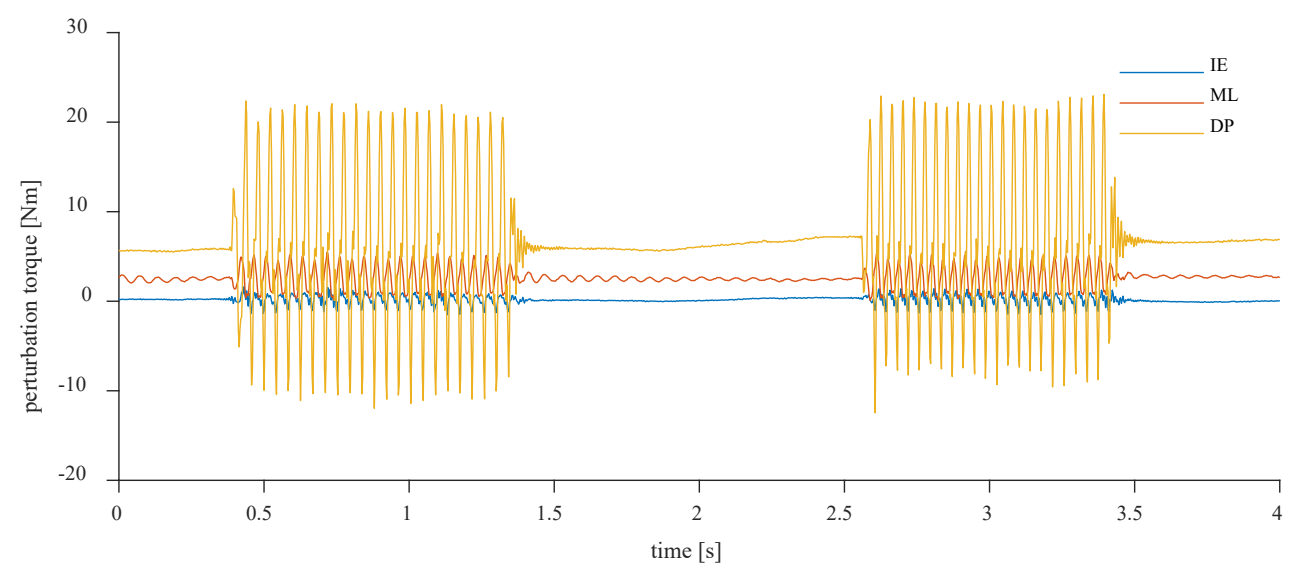

Figure 8. Example of ground perturbation. 


\subsection{Ankle Impedance Estimation}

The ankle impedance was modeled as a $2^{\text {nd }}$ order system (with stiffness, damping, and inertia), acting in parallel to the foot inertia. In addition, the ankle impedance and foot inertia were estimated for multiple rotating axes. For each rotating axis, a subset of perturbations was selected and used on a torque regression problem, resulting in the impedance parameters for that axis. Each step of this method is explained in detail next.

The external torque acting on the ankle was compensated for the force plate inertia, as described in Chapter 2. By subtracting this torque component from the calculated torque working on the subject ankle (Equation 4.2), the dynamic system can be reduced to a small inertia component (the foot) connected to a mechanical impedance (the ankle impedance).

$$
T_{F}[t] \triangleq T_{P}[t]-{ }_{0} T_{P}[t]+\left(p_{P}[t]-p_{F}[t]\right) \times\left(F_{P}[t]-{ }_{0} F_{P}[t]\right)
$$

The ankle impedance reacts to changes in the ankle angle as a $2^{\text {nd }}$ order model; In other words, it reacts with a torque proportional to a change in angle, velocity, and acceleration. The neutral position of the angle-proportional component (or stiffness) is the ankle angle in which the impedance stabilizes with zero net torque. This angle is defined as $q_{0}$ and is calculated as the ankle angle at the onset of a perturbation (Figure 9).

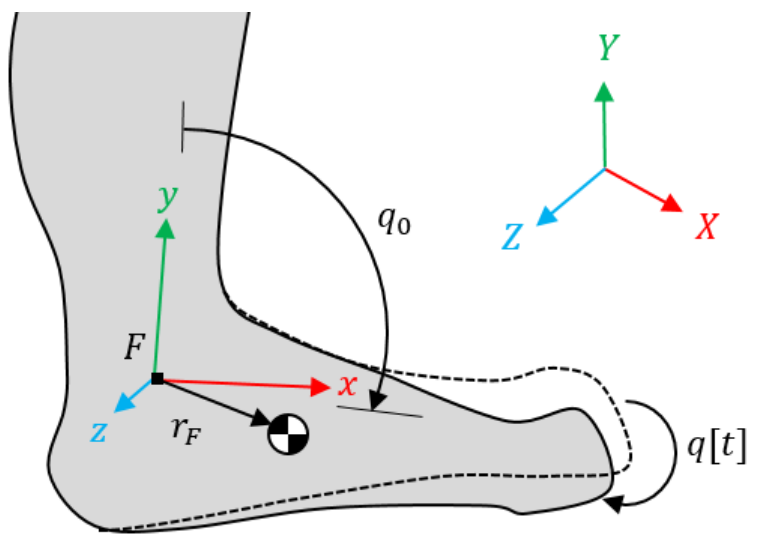

Figure 9. Coordinate frame notation for the foot. Foot origin is on the ankle center, with $x$ pointing towards the long axis of the foot, parallel to the ground, and y pointing upwards. The orientation of the foot in respect to the shank is composed by a mean angle $q_{0}$ and a small rotation $q[t]$ due to the ground perturbation. 
To calculate the ankle angles during the perturbation, the orientation between the shin and foot, represented as a rotation matrix, $R_{F}[t]=R_{S}[t] R_{q_{0}} R_{q}[t]$, is decomposed into two transformations: the neutral position rotation matrix, $R_{q_{0}} \in S O(3)$, at a time $t_{0}$, from the onset of the perturbation; and a small angular displacement, $R_{q}[t] \in S O(3)$, due to the perturbation. The impedance is estimated based on this small angular displacement, $q[t]$ (Figure 10.a).

$$
\begin{aligned}
R_{q_{0}} & =R_{S}^{T}\left[t_{0}\right] R_{F}\left[t_{0}\right] \\
R_{q}[t] & =R_{q_{0}}^{T} R_{S}^{T}[t] R_{F}[t]
\end{aligned}
$$

The DP and IE ankle angles are defined as the $\mathrm{Z}$ and $\mathrm{X}$ rotations of the Euler angle (XYZ) representation of $R_{q}[t]$. The conversion between the rotation matrix to Euler angles is presented

$$
q[t]=\left[\begin{array}{c}
-\operatorname{atan}\left(R_{q}^{2,3}[t] / R_{q}^{3,3}[t]\right) \\
\operatorname{asin}\left(R_{q}^{2,3}[t]\right) \\
-\operatorname{atan}\left(R_{q}^{1,2}[t] / R_{q}^{1,1}[t]\right)
\end{array}\right]
$$

Finally, the ankle impedance was defined as a $2^{\text {nd }}$ order model and estimated for every axis of rotation, $\phi$. For this estimation, the external torque, ankle angle, and foot kinematics must be converted to a coordinate frame aligned to the axis of rotation $\phi$. This is achieved by converting the signal to the foot frame, then rotating around the Y-axis (see Figure 9 for coordinate frame definition) by an angle $\phi$. An angle $\phi$ of $0^{\circ}, 90^{\circ}, 180^{\circ}$, and $270^{\circ}$ correspond to ankle rotations on eversion, dorsiflexion, inversion, and plantarflexion, respectively.

Suppose all the variables were converted to the axis of rotation of the perturbation, the impedance is modeled as

$$
T_{F}^{x}[t] \triangleq K q^{x}[t]+B \dot{q}^{x}[t]+J \ddot{q}^{x}[t]+\beta_{1} \dot{\omega}_{F}^{z}[t]+\beta_{2} \ddot{s}_{F}^{z}[t]+\beta_{3} \ddot{s}_{F}^{y}[t]
$$

where $K, B$, and $J$ are the stiffness, damping, and inertia, respectively. $\dot{\omega}_{F}$ and $\ddot{s}_{F}$ are the angular and linear acceleration of the foot. The operators with the superscript $*^{x}, *^{y}$ and $*^{z}$ select the $x, y$, and $z$ scalar components from the $\mathbb{R}^{3}$ vectors, respectively. In addition, because the foot inertia is numerically small [65] compared to the impedance components, only the angular and linear 
acceleration acting on the plane of motion were considered in the impedance model. The parameters $\beta_{i}$ are a combination of the inertia parameters of the foot and are not included in this analysis.

Signal Processing. The impedance parameters were estimated for each subject, at each pose, and around each rotating axis, combining samples from ten perturbations $(0.8 \mathrm{~s}$ window around each perturbation, starting at the onset of perturbation), and solving via Least Square Regression to result in a single impedance parameter set. Only the ten perturbations whose axes of rotation were the closest to the ankle rotation in question were used. To reduce the effects of lowfrequency active ankle torque and high-frequency noise in the estimation, a band-pass filter (3-35 $\mathrm{Hz}, 5^{\text {th }}$ Order Butterworth) was applied on $T_{F}, q, \dot{q}, \ddot{q}, \dot{\omega}_{F}$, and $\ddot{s}_{F}$ (Figure 10.c-10.d). All the derivatives were numerically calculated via Savitzky-Golay filter $\left(5^{\text {th }}\right.$ order polynomial in a 15 samples window). The pipeline of this impedance method is shown in Figure 10.

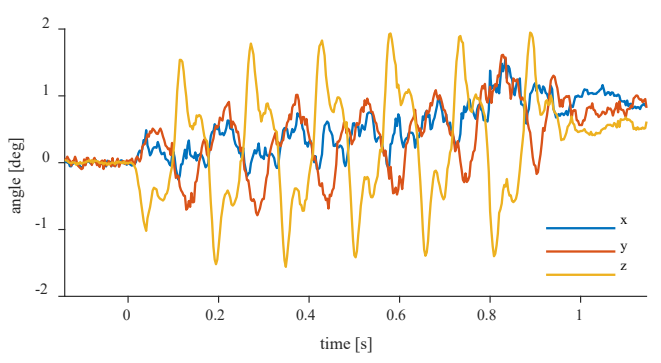

(a)

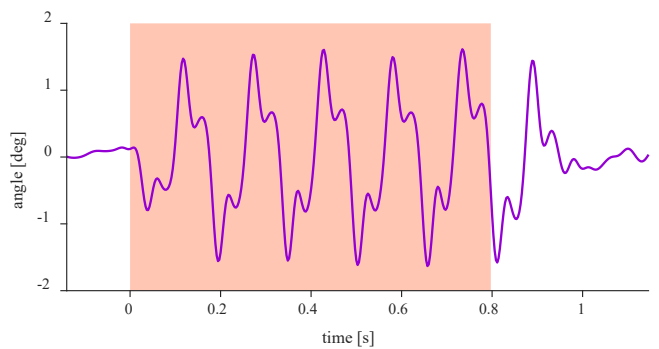

(c)

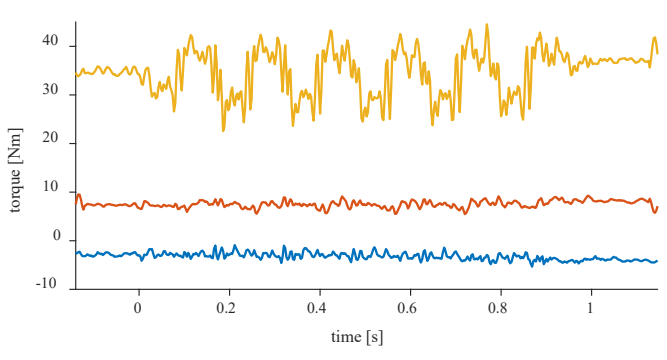

(b)

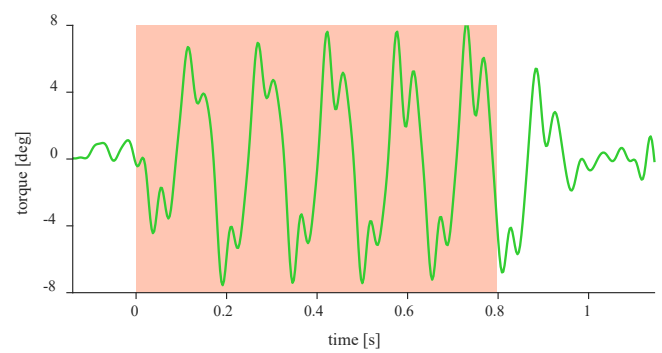

(d) 


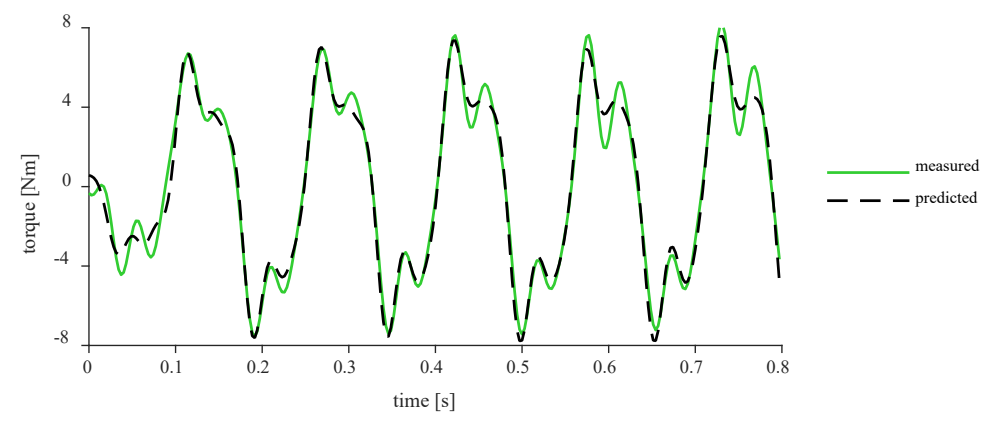

(e)

Figure 10. Stages of the ankle impedance estimation. (a) ankle angle due to the perturbation, (b) input torque acting on ankle without the FP inertia torques, (c-d) filtered angles and torques, with region of interest highlighted, and (e) the prediction of the torque using estimated ankle impedance model.

Outlier Removal. Finally, to account for the modulation of impedance due to sudden muscle contractions [66], samples with an absolute residual larger than 2.5 times the standard deviation of the residual were discarded as outliers; then the regression was recalculated. This process was repeated until there were no new outliers ( $11 \%$ of samples were rejected, and all impedance models had at least $65 \%$ inliers).

\subsection{Results and Discussion}

\subsubsection{Range of Ankle Torque and Angle}

The average CoP position in the anterior-posterior direction was $28.1 \% \pm 1.6 \%, 40.7 \% \pm 1.2 \%$, $52.7 \% \pm 2.0 \%$, and $64.9 \% \pm 2.9 \%$ of the foot length, for FF, MS, MS+, and TS, respectively. The small deviation is expected since the subjects had real-time feedback of their CoP during the experiment. The torque also increased monotonically, but with higher variance $(0.06 \pm 0.04 \mathrm{Nm} / \mathrm{kg}$, $0.25 \pm 0.03 \mathrm{Nm} / \mathrm{kg}, 0.40 \pm 0.05 \mathrm{Nm} / \mathrm{kg}$, and $0.60 \pm 0.06 \mathrm{Nm} / \mathrm{kg}$ for FF, MS, MS + , and TS, respectively) and only varied the plantarflexion torque for all poses. On the other hand, the ankle angle was in plantarflexion at FF $\left(-11.9^{\circ} \pm 3.4^{\circ}\right)$, stayed at a neutral angle for MS $\left(0.5^{\circ} \pm 3.0^{\circ}\right)$ and $\mathrm{MS}+\left(1.8^{\circ} \pm 3.7^{\circ}\right)$, and in dorsiflexion at $\operatorname{TS}\left(10.7^{\circ} \pm 2.8^{\circ}\right)$. 


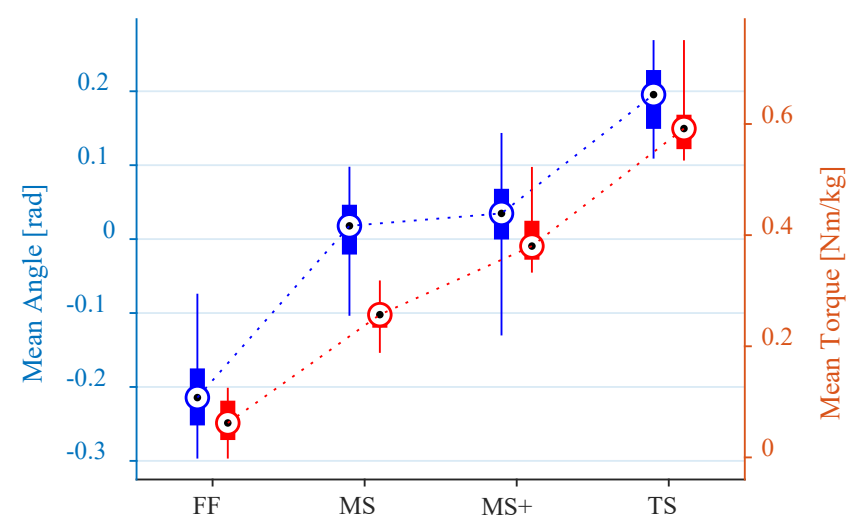

Figure 11. Mean angle and mean torque for the different stationary poses

\subsubsection{Anisotropic Ankle Impedance}

The average ankle stiffness and variance accounted for is presented in Figure 12. The DP stiffness substantially increased as the CoP moved forward, ranging from approximately $1.2 \mathrm{Nm} / \mathrm{rad} / \mathrm{kg}$ to $5.0 \mathrm{Nm} / \mathrm{rad} / \mathrm{kg}$ (4-times increase). On the other hand, the IE stiffness varied by less than $50 \%$. The invariance of IE stiffness was also noticed by Hyunglae et al. [31], [59]. In this experiment, the ankle angle did not change substantially in the IE direction. This small range of motion in IE might explain the invariance of IE stiffness. For the FF pose, the impedance was larger at the motion around 45 degrees (a combination of eversion and dorsiflexion), while for other poses the impedance was mostly symmetric across the sagittal and frontal planes. 


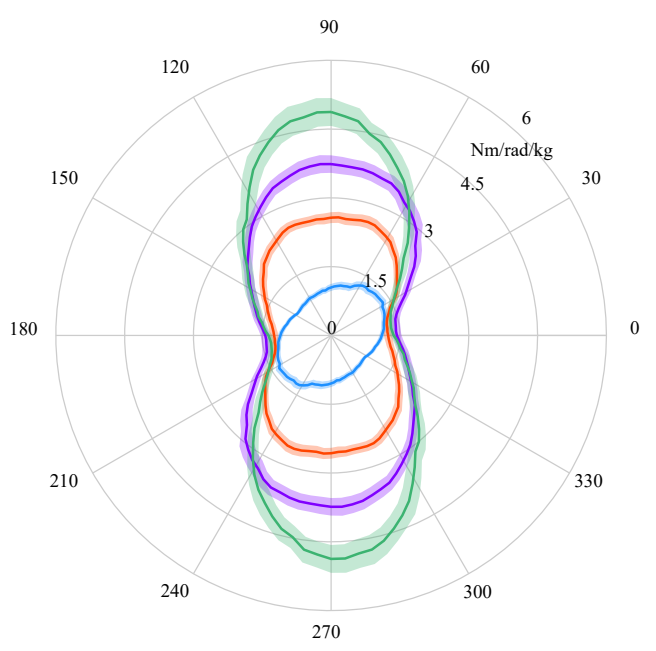

(a)

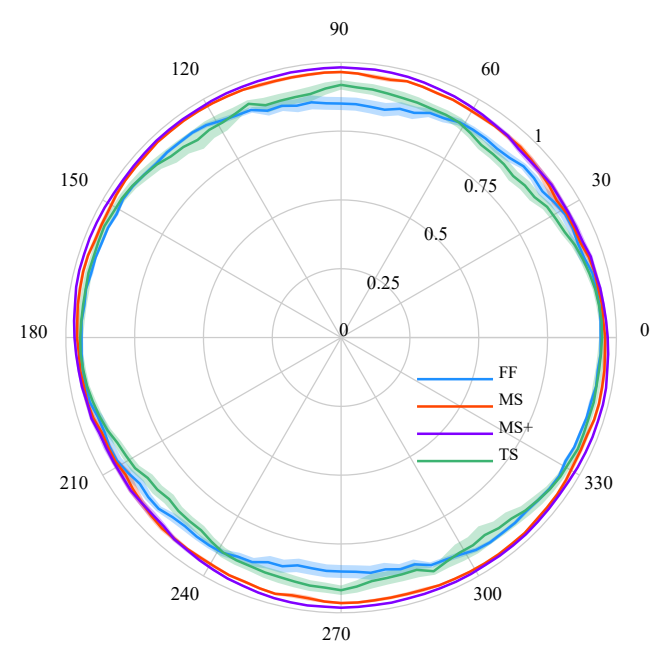

(b)

Figure 12. Anisotropic ankle stiffness for four standing poses. (a) Stiffness and (b) VAF.

The average VAF was above $90 \%$ for MS and MS+ and above $80 \%$ for FF and TS. One explanation for the lower VAF is that at the FF and TS poses, the subjects had to extend their stances in the anterior-posterior direction, making their balance less stable. To actively stabilize the stance, they had to generate an active torque around the ankle, which was not accounted on the impedance model (Equation 4.6).

The stiffness determined in this study is smaller than the respective stiffness in supine, nonloaded, ankle conditions, as reported by Weiss et al. [66]. This difference in stiffness suggests that factors other than ankle angle and torque, such as the force acting on the ankle, might affect the ankle stiffness. Alternatively, the decrease in stiffness might be caused by the active dynamics of subjects balancing in an upright pose. Future work can further understand the source of this behavior. 


\section{Time-Varying Mechanical Impedance of the Human Ankle across the Stance Phase}

\subsection{Motivation}

Since a specific group of muscles is used to move the ankle in particular directions, it is hypothesized that the impedance of the ankle depends on the direction of the ankle rotation due to an external perturbation. Therefore, the goal of this experiment was to estimate the time-varying impedance of the ankle for these different rotations. Considering that the ankle impedance depends on the axis of rotation of the ankle (see Chapter 3), sixteen axis-dependent impedance models were developed. In other words, the ankle impedance estimated in this work is a function of time and ankle axis of rotation.

\subsection{Subjects}

Four male subjects (age and Body Mass Index ranging from 25 to 31 years and $29.4 \mathrm{~kg} / \mathrm{m}^{2}$ to 25.6 $\mathrm{kg} / \mathrm{m}^{2}$, respectively) with no self-reported history of neuromuscular or biomechanical disorders were recruited for this experiment. The subjects gave written consent to participate in the experiment, which was approved by Michigan Tech's Institutional Review Board.

\subsection{Experimental Protocol}

The vibrating platform applied step function torque perturbations to the ankle along sixteen axes of rotation $\left(0^{\circ}\right.$ to $337.5^{\circ}$ in $22.5^{\circ}$ increments), in which $0^{\circ}, 90^{\circ}, 180^{\circ}$, and $270^{\circ}$ refer to ankle angle perturbations in eversion (E), dorsiflexion (D), inversion (I), and plantarflexion (P), respectively (Figure 13.b).

Ground torque perturbations in the form of step inputs acted on the ankle in sixteen axes of rotation. For each perturbation, the measurements of the ankle angle and torque were projected to the plane of rotation of the correspondent perturbation for the subsequent impedance calculation. Note that the measurements were grouped based on the perturbation direction, not overall ankle motion direction. For example, at push-off, when the ankle undergoes a fast change in $\mathrm{P}$, the D 
torque perturbation may not move the foot into dorsiflexion, but instead will reduce the amount of plantarflexion of the ankle from the unperturbed gait curve.

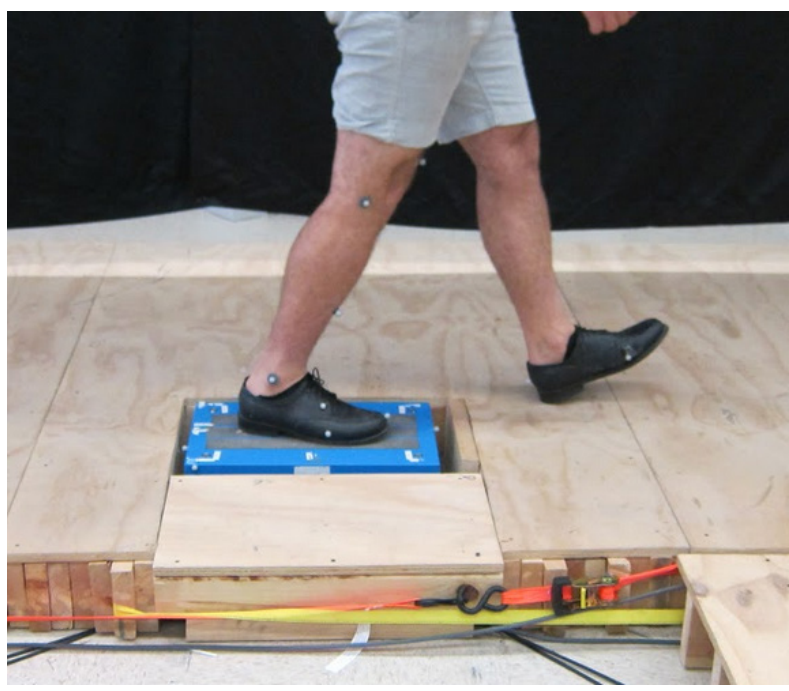

(a)

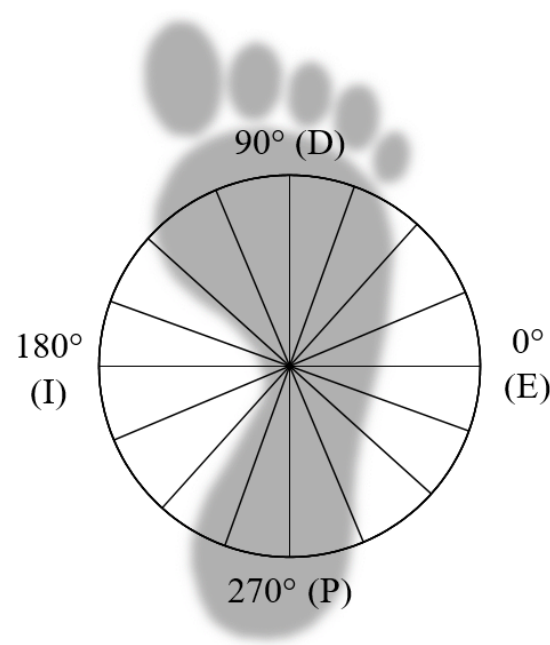

(b)

Figure 13. Axes of rotation. (a) Subject stepping on the force plate during walking experiment.

(b) Axes of rotation of perturbations applied to the ankle.

It was found, experimentally, that 100 steps at each of the 16 axes of perturbation is sufficient to converge to a result, even after removing about $6 \%$ of the steps as outliers, resulting in 1600 measured steps per subject. The experiment consisted of 16 trials of 100 steps (equally divided into 2 consecutive days of tests), with rest between trials. For each measured step, the subjects walked 3 steps on the first half of the walkway, completing their 4th step on the vibrating platform, and continued walking for 3 more steps to near the end of the walkway. Next, they turned around and repeated the procedure in the opposite direction. This walking procedure was repeated until 100 perturbed steps were measured. To reduce variations in walking speed and stance duration, a metronome was used at a pace chosen by the subject which was on average $96.6 \pm 2.2$ steps per minute.

Each perturbation occurred around a random axis (out of the possible 16 axes of rotation), in a random stage of the stance phase, to contain reflexive dynamics and preventive reactions by the subject. The vibrating platform preloads the actuators prior to the subject making contact, then actuates in full torque at a random time of the stance phase. The stance duration was measured prior in a calibration trial (for each subject). The perturbations were configured to cover the full stance cycle; therefore, it could miss the subject if the measured gait cycle was too short, too late, or too 
early. And in these cases, the failed perturbations were excluded from the impedance estimation. The average ankle rotations due to the perturbations across the 16 axes of perturbations and across all subjects were $1.2^{\circ} \pm 0.8^{\circ}$.

\subsection{Identification of the Time-Varying Ankle Impedance}

The steps were separated into 16 groups, according to the type of perturbation applied to the step, resulting in $100.0 \pm 11.1$ steps per group. The ankle angle and torques were converted to the foot coordinate frame then rotated along the foot's Y-axis (pointing up) by an angle $\phi$, then the $\mathrm{X}$-axis component of the rotated axis was selected. In other words, the torque and angle were projected to the plane of rotation. This calculation was performed on each of the sixteen groups.

$$
\boldsymbol{q}^{R}=\left[\begin{array}{ccc}
\cos \phi & 0 & \sin \phi \\
0 & 1 & 0 \\
-\sin \phi & 0 & \cos \phi
\end{array}\right]^{T} \boldsymbol{q}
$$

where $\phi, \boldsymbol{q} \in \mathbb{R}^{3}$, and $\boldsymbol{q}^{R} \in \mathbb{R}^{3}$ are the axis of rotation in question, a variable represented in the foot frame (either ankle torque or angle), and the same variable represented in the coordinate frame of the rotation.

The outlier steps were removed based on three criteria: the stance duration, the average angle, and the average torque. Each criterion was evaluated separately and the step was kept if it passed all three criteria. The stance duration, average angle, and average torque of each step had to be between the 5 and 95 percentiles of the population of recorded steps. This procedure removed less than $30 \%$ of total steps (there were many steps that failed multiple criteria, which resulted in overall fewer steps removed).

The angles and torques were low-pass filtered (20 Hz cutoff) and linearly interpolated to have the duration be equal to the average stance duration $(0.72 \pm 0.03 \mathrm{~s})$. This was done with the MATLAB's resample command, which uses a polyphase anti-aliasing filter besides performing the interpolation. The interpolation of this magnitude has been reported to affect less than $3 \%$ of the impedance estimation [25]. Next, the angular velocity, $\dot{q}$, and angular acceleration, $\ddot{q}$, were calculated numerically with a central derivative approach. 
The measured angles and torques have two components: a large curve from the gait activity and a smaller curve due to the external perturbation. The smaller component is the signal that should be used for the impedance estimation because it is the reactive component of the ankle torque. To extract the reactive components, the interpolated steps were averaged across step repetitions, resulting in the larger gait component (because the random small perturbations are averaged out). Next, from each step, the larger gait component is removed, resulting in only the reactive components. This same procedure was applied to the angular velocity and acceleration.

Another round of outlier detection removed approximately $20 \%$ of the steps. In this detection criterion, the steps with angles or torques with any sample outside of the \pm 3 standard deviation boundaries were removed (calculated across step repetitions). This resulted in $77.7 \pm 10.3$ steps per axis of perturbation. Finally, the ankle impedance was calculated for each step on a moving window (100 ms long, $20 \mathrm{~ms}$ overlap). The stiffness, damping, and inertial parameters were calculated using a constrained least-square optimization method (MATLAB's lsqnonneg function).

$$
T_{F}[t] \triangleq K q[t]+B \dot{q}[t]+J \ddot{q}[t]
$$

This procedure resulted in a time-varying impedance curve for each step. These results were averaged across step repetitions, without including time sections that are prior to or $125 \mathrm{~ms}$ after the perturbations (to remove signals with low signal to noise ratio). This averaged impedance was low-pass filtered ( $20 \mathrm{~Hz}$ cutoff) because it has been reported that $98 \%$ of the power of the ground reaction forces during gait is below $10 \mathrm{~Hz}$ and $99 \%$ is below $15 \mathrm{~Hz}$ [67].

\subsection{Results and Discussion}

The average of the stiffness and damping were calculated across the four subjects and presented in polar plots with \pm 1 standard deviation. Each parameter was shown in eight polar plots, representing increasing moments of the stance phase. The inertia was not presented because it included the effects of the foot and force plate inertia combined.

In general, the ankle stiffness and damping parameters are low at heel-strike, then they increase towards mid-stance, and decrease towards push-off. The stiffness in IE was higher than in DP from the heel-strike to about $24 \%$ of the stance phase. This might help stabilize the gait from 
rolling the ankle during the heel-strike. Over time, the DP stiffness increases (similarly to the results of Chapter 4) until about $50 \%$ of the stance phase. This increase in stiffness agrees with previous time-varying DP stiffness studies [24], [68].

Interestingly, the ankle stiffness during early stance was not symmetric as previously reported in experiments with an unloaded ankle [31], [59]. It showed the highest value along DE/PI, which are ankle rotations close to the axis of rotation of the subtalar joint. Similarly, the damping past the midstance phase was higher along this same axis. This shows that ankle impedance is not symmetrical to the anatomical axis, but mostly about the subtalar joint.

In addition, the complex shape of the stiffness, especially in early stance (stance phase $<$ $24 \%$ ), cannot be approximated with only two parameters as done in Chapter 2, in which $K_{D P}$ and $K_{I E}$ parametrized the ankle stiffness. For example, the stiffness along the DI motion is not a combination of D and I motion; But rather, an independent value. Alternatively, the stiffness can be approximated by a Fourier series with high order, as demonstrated by Ho et al. [43]. Future work can implement this multi-directional impedance in a 2-DOF powered prosthesis, such as the prosthesis developed by Ficanha et al. [11]. 

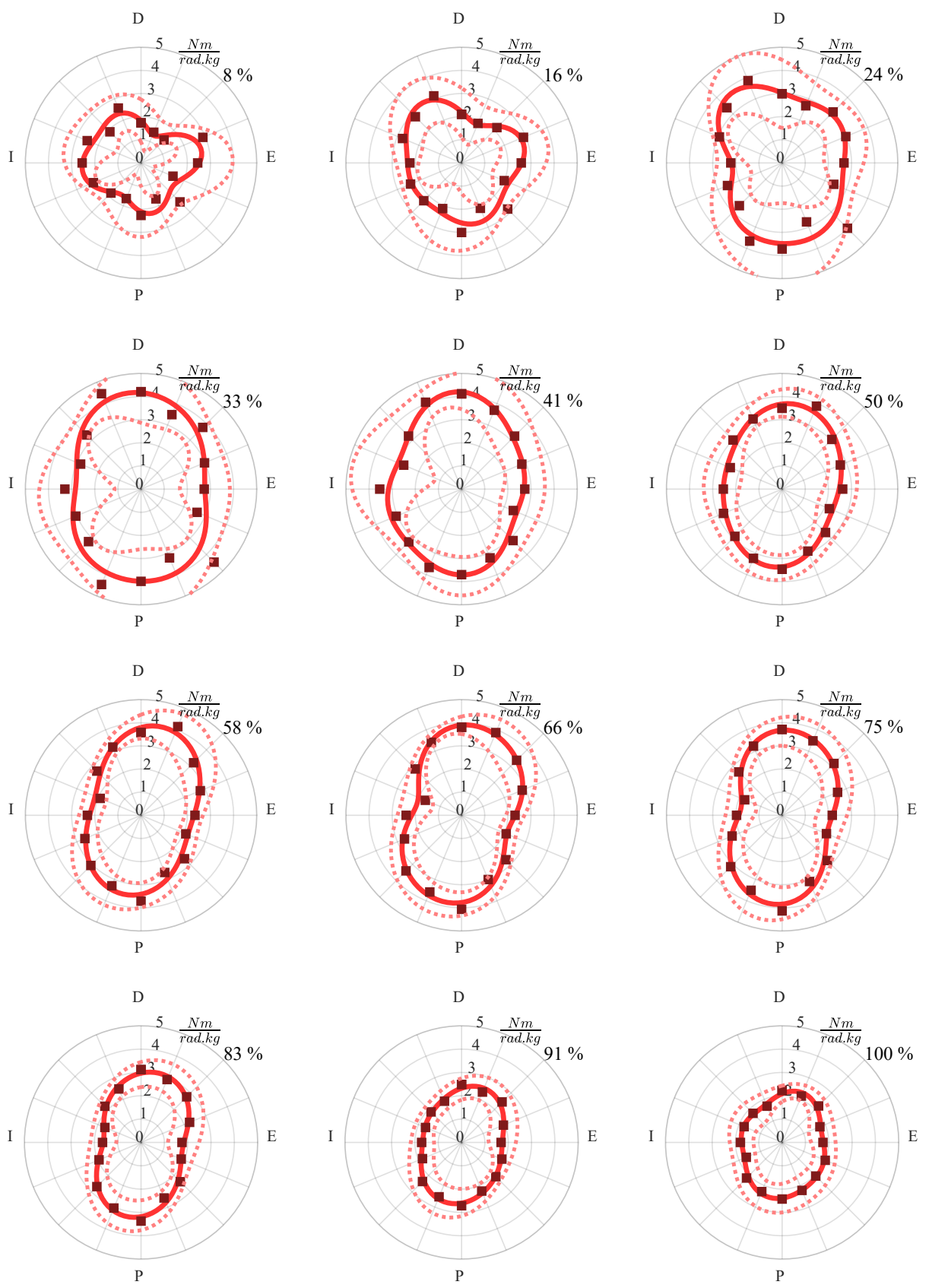

Figure 14. Average normalized time-varying ankle stiffness across the stance phase. The stiffness is normalized by the subjects' masses. The square markers, solid line, and dotted lines represent the measured stiffness, smoothed average, and smoothed \pm standard deviation, respectively. 

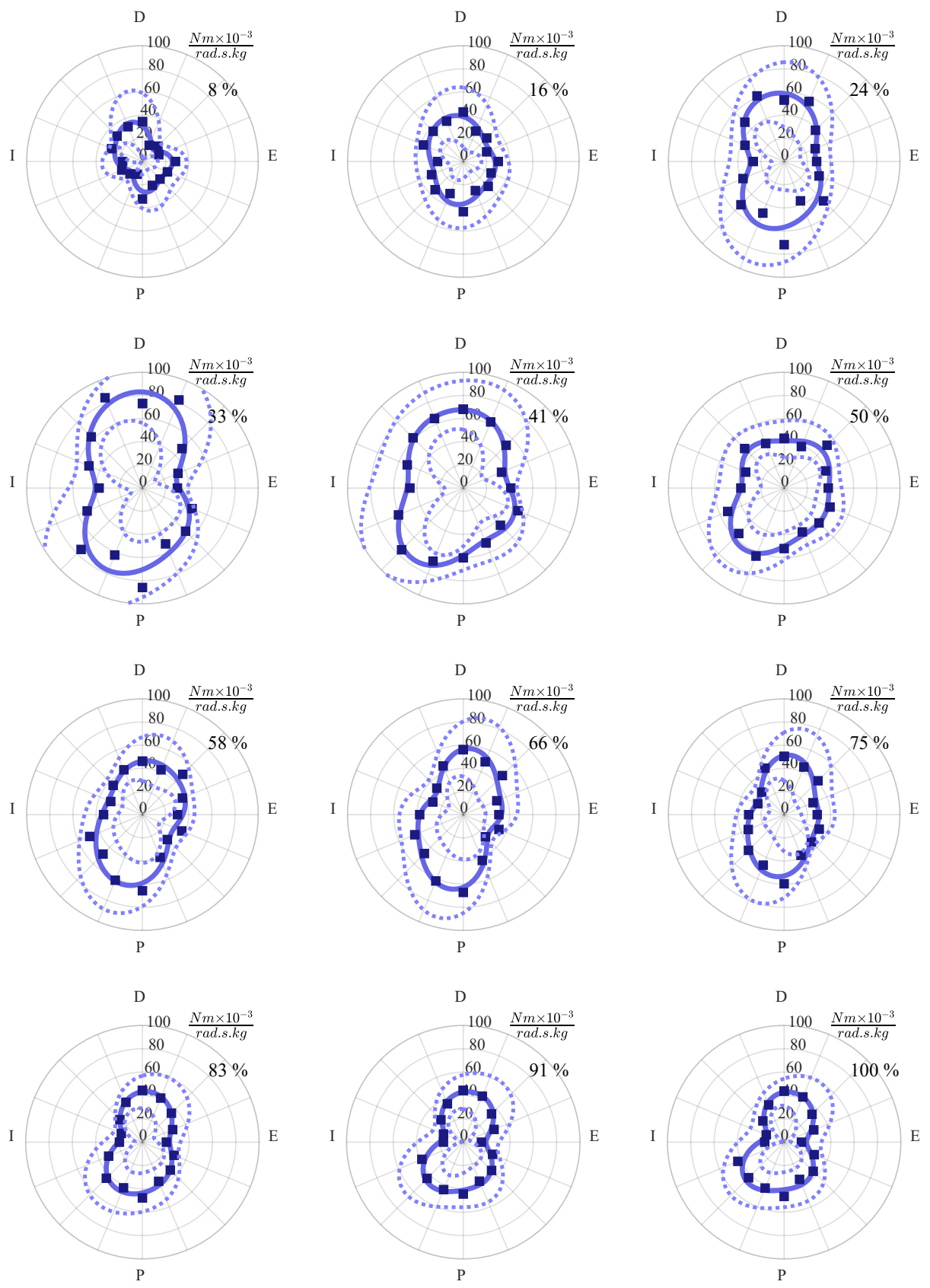

Figure 15. Average normalized time-varying ankle damping. The damping is normalized by the subjects' mass. The square markers, solid line, and dotted lines represent the measured damping, smoothed average, and smoothed \pm standard deviation. 


\section{Design of a Vision Device to Assist Impedance Modulation of Ankle-Foot Prostheses}

\subsection{Motivation}

In view of the effects of gait phase on the ankle impedance, a prosthesis control must be aware of the user's state. This chapter reports the design of a device with environment-sensing capabilities, named Vision Device for Gait Assistance (VDGA). The hardware and software components are described, and in the final sections, a calibration was performed to estimate the dimensional characteristics that are used in further chapters.

\subsection{Hardware}

The VDGA is composed of a mobile computer (Hardkernel ODROID-XU4), a supervisor computer (Laptop with Ubuntu 16.04 operational system), a depth camera (PMDTec CamBoard pico flex), a microcontroller (WeMos Lollin32/ESP32), an IMU (Adafruit BNO055), a force-sensitive resistor circuit (Interlink 402 and $5 \mathrm{k} \Omega$ ), a battery (Lithium-Polymer $5200 \mathrm{mAh}, 60 \mathrm{C} 11.1 \mathrm{~V}$ ), and a voltage regulator (uxcell $12 \mathrm{~V}$ to $5 \mathrm{~V}, 50 \mathrm{~W}$ ). The subject wears the device, with all the instrumentation, while the state and measurements of the device can be visualized on the supervisor computer. The device has two modules: a light-weight shank shell $(0.13 \mathrm{~kg})$, holding all the sensors; and the waist pack, holding the mobile computer and battery circuit $(0.78 \mathrm{~kg}$, in which $0.40 \mathrm{~kg}$ is the battery). Note that the mobile computer does not have a monitor or keyboard to allow mobility to the VDGA user; thus, requiring the remote supervisor computer to supervise and control the device. 


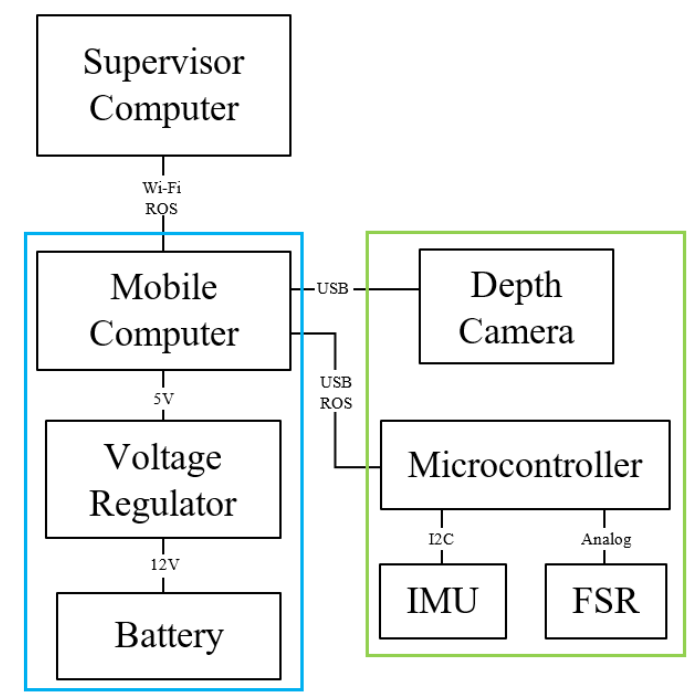

Figure 16. Components of the vision device used for gait assistance.

The shank shell must provide a stable attachment between the subject's shank and the sensors (camera and IMU) so that the measurements are consistent across time. The shank shell holds the depth camera via a camera adaptor with an adjustable pitch angle that can change the view angle of the device. Because the orientation of the camera relative to the shank shell can change, the IMU was directly attached to the body of the camera, rather than on the shank shell. The microcontroller was also attached on the shank shell, rather than on the waist pack, to reduce the length of the wires that communicate to the IMU and the force-sensitive resistor (FSR). This was preferred because the analog and $\mathrm{I} 2 \mathrm{C}$ communication are more vulnerable to environmental noise than the USB interface. Cable clips reduce the cable tension on the USB connectors and the wire tension on the microcontroller pins. The microcontroller is connected to the IMU (via four 28 AWG wires), to the FSR (via two $22 \mathrm{AWG}$ wires), and to a $50 \mathrm{k} \Omega$ resistor, directly soldered to the board. The FSR is attached on the top surface of the shoe insole, around the heel region, within two layers of athletic tape (Hampton Adams, cotton-based). 


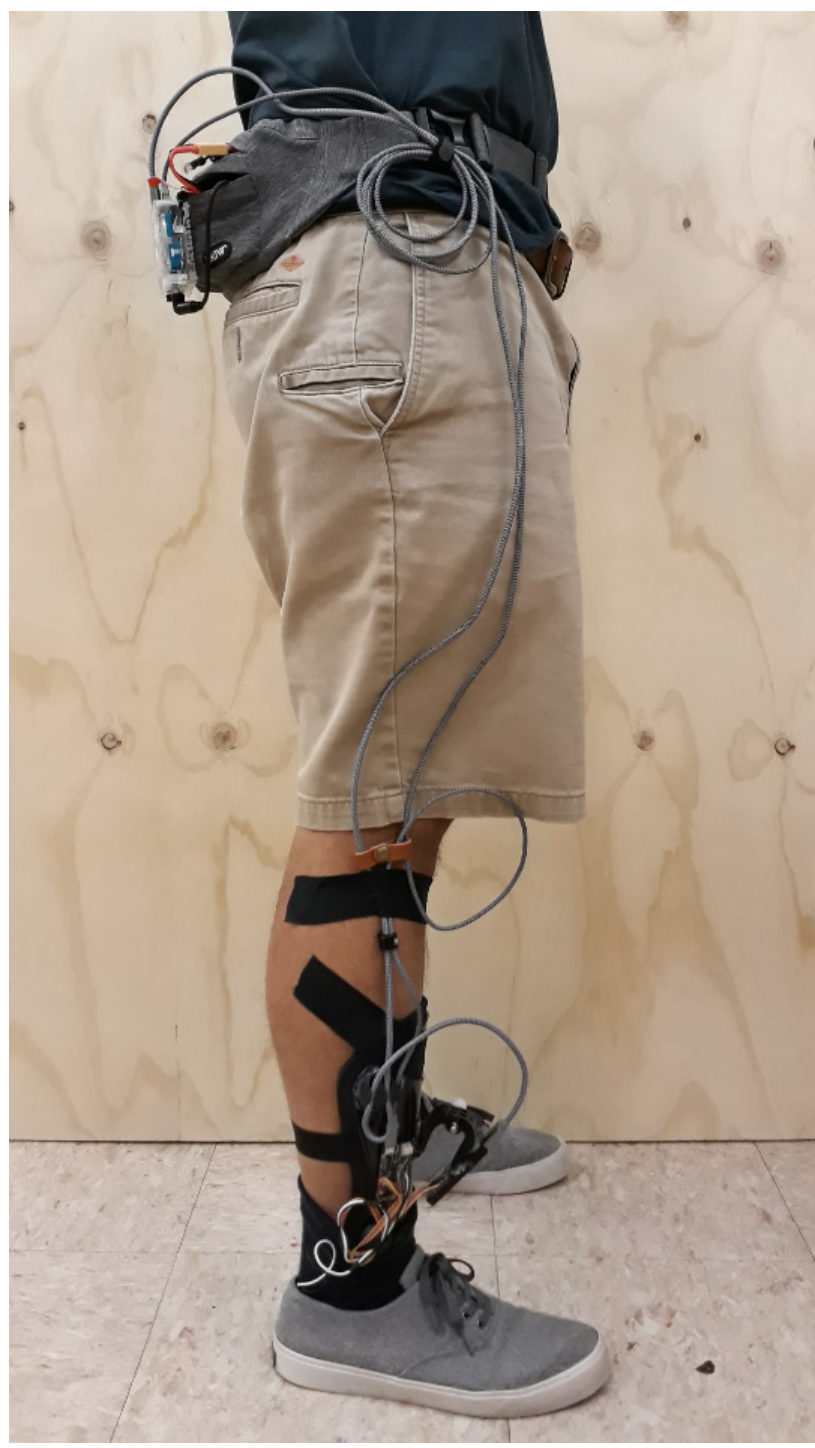

(a)

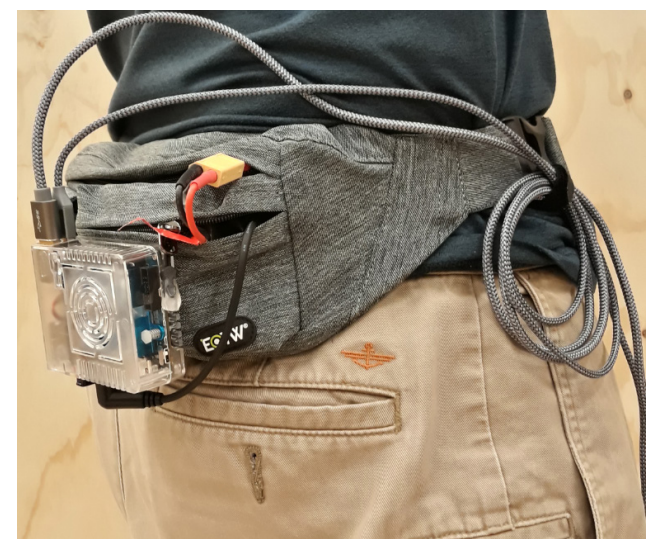

(b)

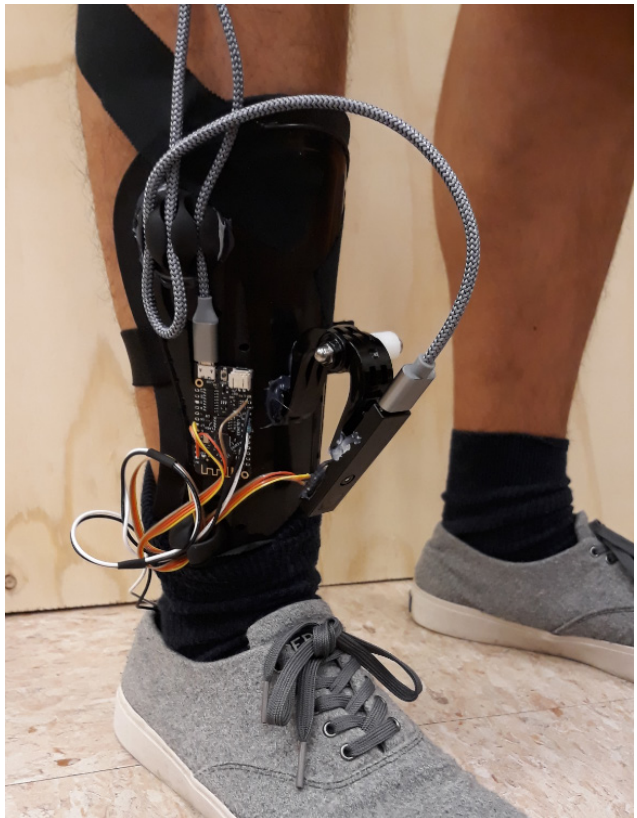

(c)

Figure 17. (a) Visual Device for Gait Assistance. (b) Waist pack and (c) shank shell.

The waist pack holds the onboard computer, the battery, the voltage regulator, and a battery voltage checkers (to prevent harm to subject or device due to battery under-voltage). The two USB cables loop around the cable clips on the shank shell, then are taped to the leg, circle around the cable clips on the waist pack, then connect to the onboard computer. The use of cable clips and cable tapes to the subject's leg prevent damaging the connectors and reduce the discomfort of loose cables, which can substantially impact the normality of the gait during experiments. Finally, the onboard computer was stitched on the outside of the waist pack, rather than stored inside of it, to improve airflow and minimize overheating. 


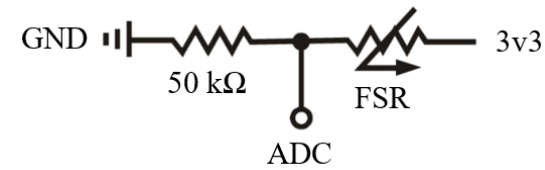

Figure 18. FSR circuit to detect the stance phase. When the FSR is under mechanical load, the electrical resistance decreases, and the ADC detects a voltage increase.

The FSR and the $50 \mathrm{k} \Omega$ resistors are connected in series to form a voltage divider circuit, allowing the microcontroller to measure the change in resistance of the FSR in the form of voltage change (Figure 18). The common terminal of the resistors (middle tap) is connected to the analog to digital converter (ADC) of the microcontroller with 12 bits of resolution, while the other FSR terminal connects to the positive microcontroller reference $(3.3 \mathrm{~V})$ and the additional resistor connects to the negative reference $(0 \mathrm{~V})$. When the FSR is under mechanical pressure, which occurs when the VDGA wearer has the foot heel loaded, the FSR resistance drops from the nominal $1 \mathrm{M} \Omega$, and the microcontroller's ADC senses a rise on the middle tap voltage.

\subsection{Software Architecture}

The onboard computer was configured as a Wi-Fi (802.11ac) hotspot to share sensor measurements and remote access to the clients on the network (the supervisor computer) from a radius of more than 10 meters. Because the onboard computer does not have a monitor or an input device, the supervisor computer must manage the VDGA (e.g., starting programs, displaying the device status and measurements, turn-off device). The onboard computer, the supervisor computer, and the microcontroller are all integrated into the same software platform called Robotic Operational System (ROS).

ROS is a growing platform used in robotics because it facilitates development and integration of software. ROS provides an infrastructure for multiple programs (ROS nodes), executing in real-time, and possibly on different computers to exchange information such as sensor measurements (ROS messages), device statuses, and action requests. Because it standardizes the communication between programs, the software library developed by a third party (ROS packages) can be easily reused into a new system. Consequently, it aggregates a large community of developers focused on integration and documentation of their work. The main reasons the VDGA software was developed on ROS was to 1) Synchronize the measurements from the camera, IMU, 
and force pressure sensors; 2) Transfer IMU and force pressure data from the microcontroller to the mobile computer; 3) Visualize the sensor data and status in real-time on the supervisor computer; 4) Use image processing and point cloud libraries developed by a third party; 5) Store sensor measurements for post-processing on another platform; and 6) Facilitate the development of future work of the VDGA by other researchers.

The graph of the programs and information on the VDGA system (mobile and supervisor computers) are shown in Figure 19, where the ROS nodes are represented as ellipses, the ROS messages are the boxes, and the flow of information is represented by the arrows. The mobile computer executes the nodes: /camera_driver, /plane_estimator, /serial_arduino, and /logger. The node /camera_driver communicates with the depth camera using the Royale Software Development Kit (SDK) and ROS driver provided by the manufacturer [69]. It publishes the depth images, /depth, the intrinsic calibration of the camera, /cam_info, and the infrared intensity images, /intensity. The node/serial_arduino emulates the communication of the microcontroller to the entire ROS network as if it were a ROS-enabled computer. The /serial_arduino node and the program executing on the microcontroller collaborate to synchronize and publish the IMU measurements, /imu, and the voltage across the FSR, /heel_pressure. The messages /imu, /depth, and /cam_info are used by the node /plane_estimator to fit the ground profile to a plane model, /plane. For post-processing, the node /logger records the messages /plane, /depth, /cam_info, /intensity, /imu, /heel_pressure, and /plane. On the supervisor computer, the nodes /rviz and /plot display the intensity image and the /heel_pressure, respectively. 


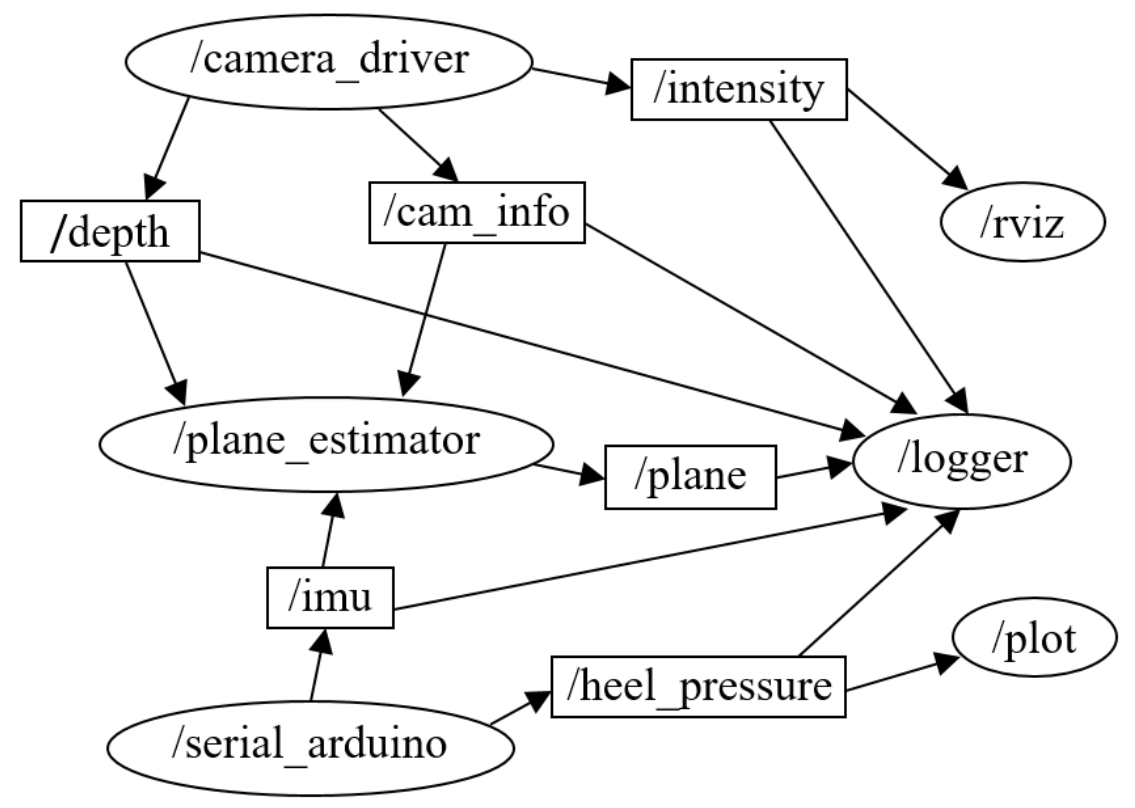

Figure 19. Graph of the processes executing on the VDGA system. Ellipses are ROS nodes, boxes are ROS messages, and the arrows point to the subscriber to the messages.

The microcontroller publishes the IMU and FSR measurements to the mobile computer via serial communication (UART, 500000 Baud rate). The interface to the IMU uses the Adafruit BNO055 library [70] and physically via the I2C serial protocol (400 kHz clock), providing a data rate of $100 \mathrm{~Hz}$. The heel force is measured at the same rate, synchronized with the IMU.

\subsection{Extrinsic Calibration between the Camera and the IMU}

Figure 20 shows the VDGA shank shell with the depth camera, the IMU sensor, a visual landmark, and the inertial frame from which the IMU is oriented (in which $\mathrm{X}, \mathrm{Y}$, and $\mathrm{Z}$ axes point to east, north, and up, respectively). To represent environmental measurements independent of the VDGA orientation, the visual measurements must be converted from the camera to the inertial frame, assisted by the IMU. In addition, there is an unaccounted-for time delay between the IMU and the camera samples that arise from an accumulation of time delays (from processing time, filtering delay, systematic time-stamping error). This time delay must be known in order to be compensated for. 


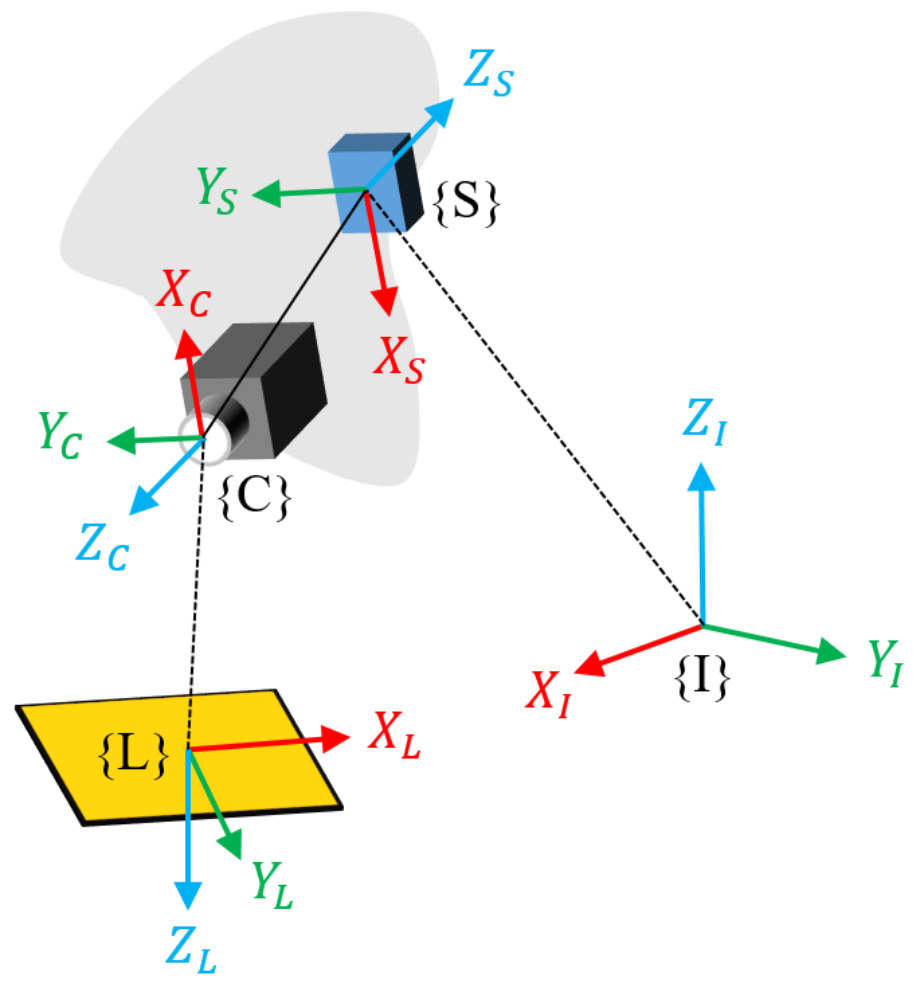

Figure 20. Coordinate frame definitions of the VDGA. A visual landmark, $\{\mathrm{L}\}$, is observed by the camera, in the camera frame $\{C\}$. Variables represented in this frame must be converted to the inertial frame, $\{\mathrm{I}\}$, via the IMU sensor orientation.

A spatial vector represented in the camera frame, ${ }^{\{C\}} \boldsymbol{s} \in \mathbb{R}^{3}$, is converted to the inertial frame, ${ }^{\{I\}} \boldsymbol{s} \in \mathbb{R}^{3}$, via the coordinate transformation

$$
\begin{gathered}
{\left[\begin{array}{c}
0 \\
\{I\}
\end{array}\right]=\bar{q}_{C} \otimes\left[\begin{array}{c}
0 \\
\{C\}
\end{array}\right] \otimes q_{C}} \\
q_{C}=q_{S} \otimes \bar{q}_{C S}
\end{gathered}
$$

where $q_{C} \in S O(3), q_{S} \in S O(3), q_{C S} \in S O(3)$ are the unit quaternions representing the rotation of the camera, the IMU sensor, and the IMU sensor relative to the camera, respectively. The operators $\otimes$ and $\bar{q}$ are the quaternion multiplication and conjugate, respectively. The IMU sensor used in this device has an embedded microcontroller that estimates the absolute orientation of the sensor and 
compensates for the bias noise of the measurements [71]. Therefore, $q_{S}$ is known but $\bar{q}_{C S}$ must be estimated via an extrinsic calibration procedure.

There are many extrinsic calibration approaches [72], [73] reported in the field, however, because the IMU BNO055 provides the sensor orientation directly and the noise biases are not easily quantifiable (due to the bias noise compensation performed by the embedded microcontroller), an ad hoc solution was preferred. This solution is similar to standard approaches, in which it also uses a visual landmark to estimate the camera motion, resulting in the relative orientation between devices and the time delay between visual and inertia measurements. Different from standard approaches, this solution also uses the IMU orientation together with the angular velocity and linear acceleration.

\subsubsection{Calibration Procedure}

As shown in Figure 21, the VDGA was moved across space, exciting all three axes (Figure 21.a), while the depth camera focused on a visual landmark (a checkerboard, $5 \times 6$ internal grids, grid length of $30 \mathrm{~mm}$, Figure 21.b). The intensity images, camera intrinsics, and the IMU measurements were recorded for post-processing on a MATLAB script. The pose of the camera with respect to the landmark was estimated (function detectCheckerboardPoints), given the undistorted intensity images, camera intrinsic properties, and checkerboard dimensions.
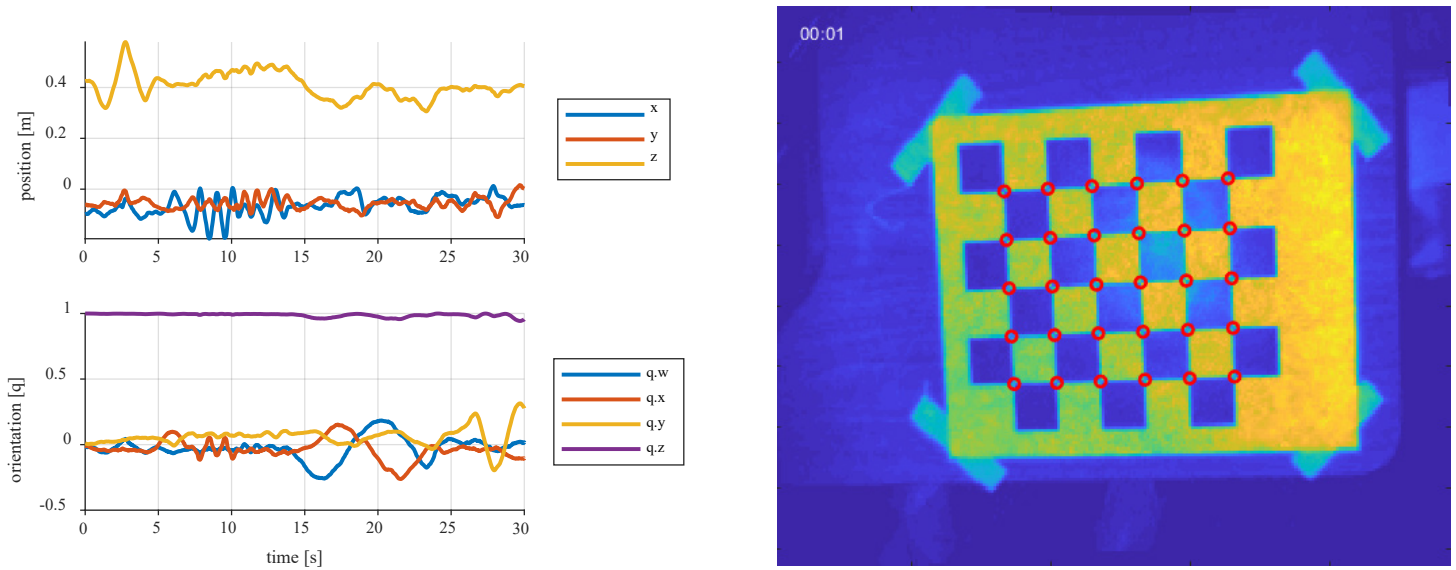

(a)

(b)

Figure 21. Intrinsic calibration setup. (a) Position and orientation of the camera with respect to the (b) visual landmark (checkerboard). 
The calibration procedure involves predicting the IMU orientation $q_{S}$, body angular velocity, ${ }^{\{S\}} \boldsymbol{\omega}$, and linear body acceleration (with the static acceleration artifact from the gravity), ${ }^{\{S\}} \boldsymbol{a}_{S}$, given the camera motion and the unknown extrinsic parameters. These variables are calculated as

$$
\begin{gathered}
q_{S}(t)=q_{I L} \otimes q_{L C}\left(t+t_{d}\right) \otimes q_{C S} \\
{\left[\begin{array}{c}
0 \\
\{S\} \\
\boldsymbol{\omega}(t)
\end{array}\right]=q_{C S} \otimes\left[\begin{array}{c}
0 \\
\{C\} \\
\boldsymbol{\omega}\left(t+t_{d}\right)
\end{array}\right] \otimes \bar{q}_{C S}} \\
\boldsymbol{a}_{S}(t)=\boldsymbol{a}_{C}\left(t+t_{d}\right)+{ }^{\{C\}} \dot{\boldsymbol{\omega}}\left(t+t_{d}\right) \times \boldsymbol{r}_{C S}+{ }^{\{C\}} \boldsymbol{\omega}\left(t+t_{d}\right) \times\left({ }^{\{C\}} \boldsymbol{\omega}\left(t+t_{d}\right) \times \boldsymbol{r}_{C S}\right)-\boldsymbol{g}
\end{gathered}
$$

where $q_{I L}$ is the rotation from the inertial to the landmark frame;

$q_{L C}(t)$ is the rotation from the landmark to the camera frame;

$q_{C S}$ is the rotation from the camera to the IMU frame;

${ }^{\{C\}} \boldsymbol{\omega}(t)$ is the body angular velocity represented in the camera frame;

$\boldsymbol{a}_{C}(t)$ is the linear acceleration of the camera;

$\boldsymbol{r}_{C S}$ is the position vector from the camera to the IMU, in the camera frame;

$\boldsymbol{g}$ is the gravity vector, in the inertial frame;

$t_{d}$ is the unaccounted-for time delay between the IMU and camera samples.

For simplicity, the quaternion operations on Equation 7.5 were suppressed. But as a note, $\boldsymbol{a}_{C}(t)$ and $\boldsymbol{g}$ are converted from the landmark and inertia frames, respectively, to the IMU frame. The other components of the equation are converted from the camera to the IMU frame.

The unknown parameters $q_{I L}, q_{C S}, r_{C S}$, and $t_{d}$ were estimated by minimizing the residual of Equations 7.3, 7.4, and 7.5, with weighting factors of 0.2, 5.0, and 10.0, respectively. The weighting factors makes the units of angle, angular velocity $(\mathrm{rad} / \mathrm{s})$, and linear acceleration $\left(\mathrm{m} / \mathrm{s}^{2}\right)$ numerically similar amongst each other. The residuals of Equations 7.4-7.5 were computed as mean 
squared errors, while the residual of Equation 7.3 was the mean absolute angle between the calculated and measure quaternions. The camera pose was upsampled to the rate of the IMU, at 100 $\mathrm{Hz}$; its time-derivatives were calculated with a Sarvitzky-Golay filter [51] (with a $3^{\text {rd }}$ order polynomial on 21 points), and its time-shifts were calculated with spline interpolation. Finally, the problem was minimized with MATLAB's fmincon function (interior-point algorithm [52]) with multiple starting points (MATLAB's MultiStart algorithm) because this problem has shown to have multiple local minima.

\subsection{Results and Discussion}

The extrinsic calibration estimated the $q_{C S}=[-0.0123,0.9997,0.0213,0.0016]$ (x, y, z, w components, respectively) and $t_{1}=34.9 \mathrm{~ms}$ (the reconstruction of all the IMU measurements are shown in Figure 22). The $q_{C S}$ parameter converged to an expected value, agreeing with the sensor axes described by the manufacturer (the IMU and camera orientation is depicted in Figure 20). The mean absolute error of the IMU orientation was 0.66 degrees.
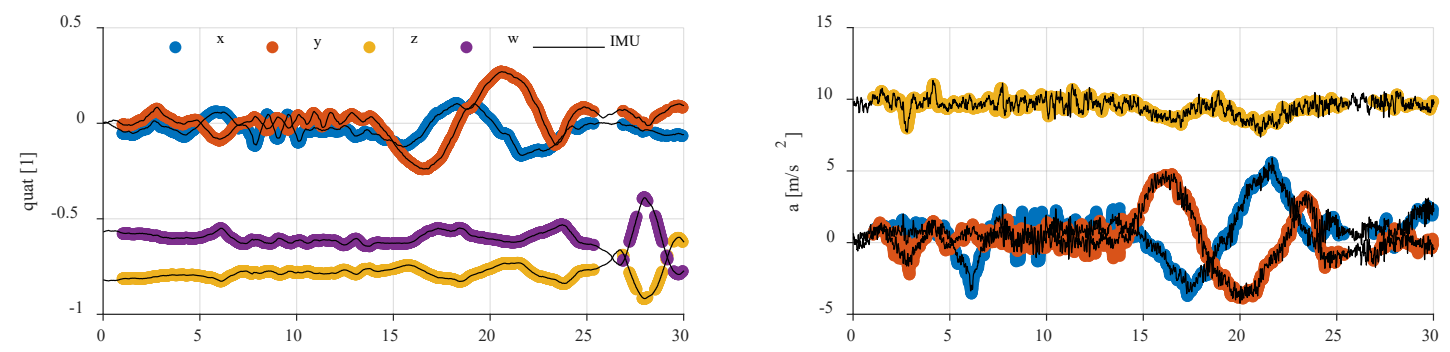

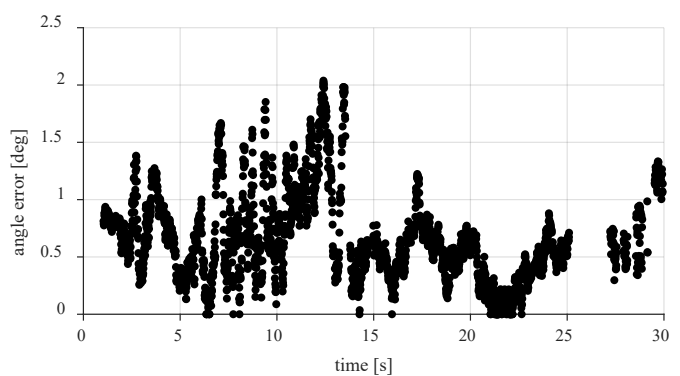

(a)

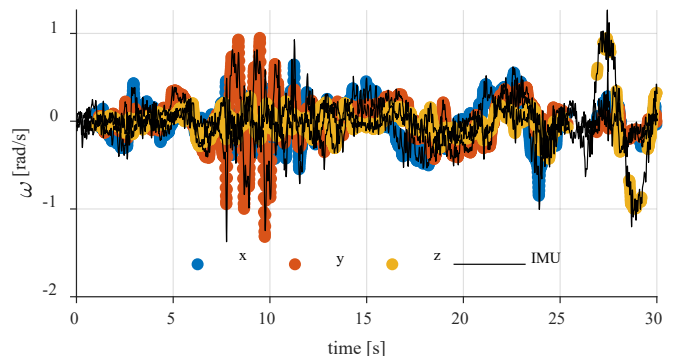

(b)

Figure 22. IMU orientation, linear acceleration, and angular velocity during the extrinsic calibration experiment. The IMU measurements are represented in black lines, while the colored, 
thicker, lines are the IMU predictions based on the extrinsic model and camera motion. The bottom left plot shows the quaternion error between prediction and measurements.

The parameter $q_{I L}$ was evaluated to equal $[0.8339,-0.5520,0.012,0.0003]^{T}$, which agrees with the orientation of the checkerboard, and the $r_{C S}$ parameter did not converge to an expected value. The estimation of $r_{C S}$ possibly could be improved with a higher IMU angular acceleration during the calibration procedure. However, the $r_{C S}$ is not relevant in the next analyses because it is not used to convert landmarks from the camera to the inertial frame; therefore, it is not necessary to estimate it accurately. 


\section{Heel Strike Prediction for a Prosthetic Device using Depth Vision}

\subsection{Motivation}

This chapter reports a preliminary evaluation of the VDGA sensing accuracy. The capabilities to 1) sense the environment by estimating the ground inclination and 2) to predict the user intent by estimating the gait phase are described in Section 7.2 and evaluated in Section 7.3.

\subsection{Methods}

\subsubsection{Environment Characterization}

The VDGA characterizes the environment as a plane, representing the ground, whose parameters are represented with respect to the inertial frame. The plane parameters with respect to the camera frame are first estimated with the depth camera, then converted to the inertial frame with the estimated IMU orientation.

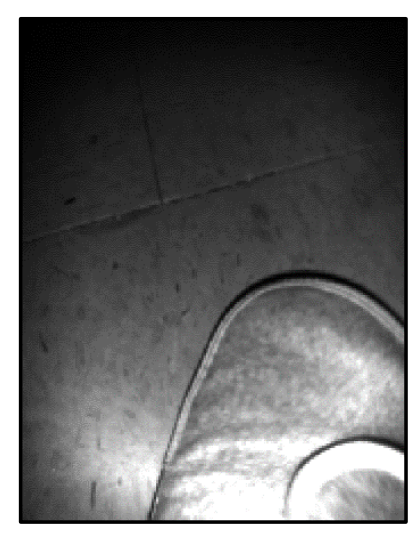

(a)

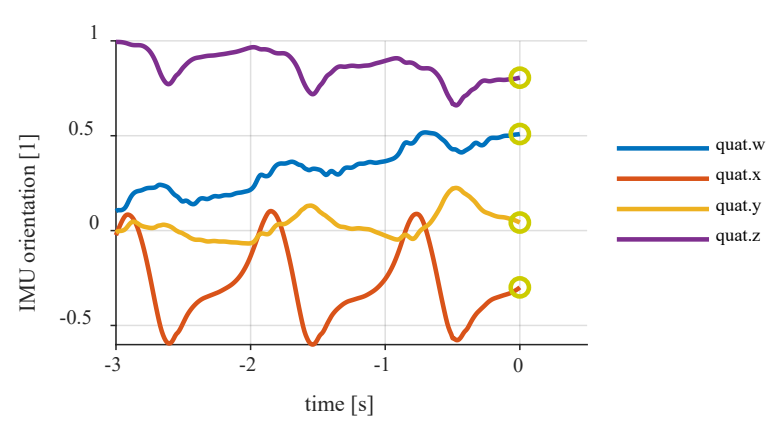

(b)

Figure 23. Measurements from the VDGA during walking, showing the ground and a section of the subject's shoe. Depth camera (a) intensity and (b) IMU orientation represented as a unit quaternion. 
The depth camera also captures objects above the ground, including a section of the subject's foot (Figure 23.a) and possible obstacles on the ground. These objects must be recognized as outliers so the algorithm can characterize the ground plane without bias errors. This is achieved with the Random Sample Consensus (RANSAC) algorithm, which searches for a set of inlier points that best explain the identified model [74]. This algorithm is implemented with the Point Cloud Library [75], involving the following steps: A) the point cloud is downsampled into a voxel grid with a resolution of $30 \mathrm{~mm}$ (Figure 24.b), which decreases the execution time of the process; B) discard points more than one meter in front of the camera to avoid estimating planes from points that are far away from the subject (which can happen during final swing, when the camera pitch angle is high); C) calculate the normal vector of each neighboring (100 mm radius) set of downsampled points; D) apply the RANSAC algorithm on the downsampled points and normal vectors to fit a plane in which the inlier points have similar normal vectors and lie within $50 \mathrm{~mm}$ of the plane.

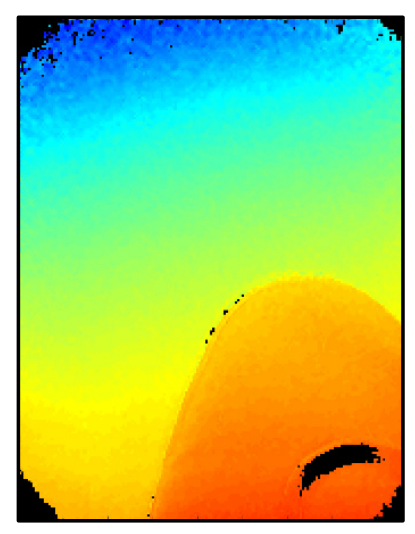

(a)

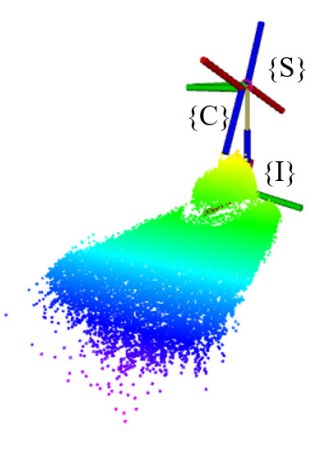

(b)

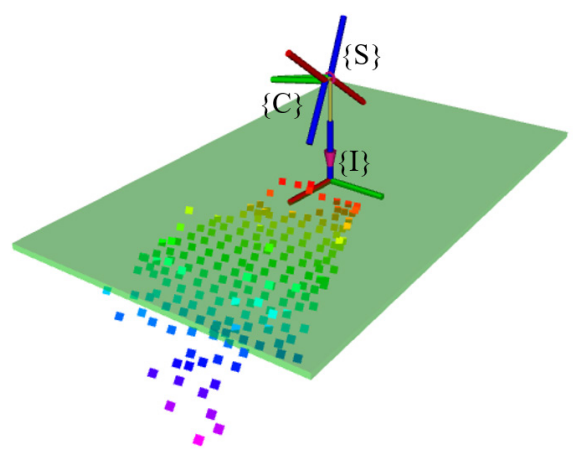

(c)

Figure 24. Point cloud processing pipeline. Convert depth image (a) into point cloud (b), downsample cloud into a voxel grid, and estimate plane from points close to plane and with low angular deviation (c). $\{\mathrm{C}\},\{\mathrm{S}\}$, and $\{\mathrm{I}\}$ are the coordinate frames fixed on the camera, IMU sensor, and inertial frame, respectively. The $\mathrm{X}, \mathrm{Y}$, and $\mathrm{Z}$ axes are shown in red, green, and blue colors, respectively.

The resulting plane model from the RANSAC algorithm is represented by a mathematical model (Equation 7.1) in which the origin is the optical center of the camera and the $\mathrm{X}, \mathrm{Y}$, and $\mathrm{Z}$ 
axes point upwards, to the right, and forward from the image perspective, respectively (Figure 24.a).

$$
a x+b y+c z+d=0
$$

where $a, b, c$, and $d$ are the coefficients of the plane model. However, a more useful representation of the plane is through the normal vector to the plane ( $\widehat{\boldsymbol{n}}$, Equation 7.2$)$ and the plane distance to the camera ( $p$, Equation 7.3), calculated as follows

$$
\begin{gathered}
\widehat{\boldsymbol{n}}=\left[\begin{array}{lll}
a & b & c
\end{array}\right]^{T} / \sqrt{a^{2}+b^{2}+c^{2}} \\
p=d / \sqrt{a^{2}+b^{2}+c^{2}}
\end{gathered}
$$

\subsubsection{Gait Cycle Estimation}

As shown in previous sections, the mechanical impedance varies across the gait phase due to a combination of reasons (muscle contraction, ankle angle, and ankle torque, among others). Thus, it is essential for a powered prosthesis to accurately track the gait phase so the prosthesis can modulate the impedance accordingly. In this section, the gait phase was estimated using two approaches: a standard approach using measurements from the IMU data, and a second using measurements from both IMU and depth camera.

The response variable was labeled using the FSR measurements. Suppose the gait phase, $\varphi[t] \in \mathbb{R} \mid 0 \leq \varphi<1$, is defined as 0 on the moment of heel strike and continually increases to 1 at the heel strike of the next step. For any time, $t_{i}$, that the heel strike occurred, the FSR reading increased to above $2.1 \mathrm{~V}$ and $\varphi\left[t_{i}\right]$ was labeled as 0 . The consecutive heel strikes were linearly interpolated according to time, that is, $\varphi\left[t_{i}+t\right]=t /\left(t_{i+1}-t_{i}\right)$ for $0 \leq t<t_{i+1}-t_{i}$. In addition, to avoid the discontinuity between heel-strikes (from 1 to 0 , which hindered the regression performance), the gait phase variable was transformed into a phasor form, $\mathbb{Y}$, with two response variables:

$$
\mathbb{Y}[t]=[\cos 2 \pi \varphi[t] \quad \sin 2 \pi \varphi[t]]^{T}
$$


For the predictor variables, two approaches were tested to evaluate the benefit of using the camera depth information. The predictor variable of the first approach, $\mathbb{X}_{a}$, used the instantaneous measurements from the IMU (angular velocity and linear acceleration):

$$
\mathbb{X}_{a}[t]=\left[\boldsymbol{\omega}_{S}^{T}[t] \boldsymbol{s}_{S}^{T}[t]\right]^{T}
$$

While the predictor variable of the second approach, $\mathbb{X}_{b}$, also used the instantaneous measurement of the IMU and, in addition, the time-interpolated ground estimates: the estimated normal vector of the ground (in the camera frame) and the camera distance to the ground.

$$
\mathbb{X}_{b}[t]=\left[\begin{array}{llll}
\boldsymbol{\omega}_{S}^{T}[t] & \boldsymbol{s}_{S}^{T}[t] & \widehat{\boldsymbol{n}}^{T}[t] & p[t]]^{T}
\end{array}\right.
$$

Each phasor component of the response variable was fit individually using a Least Square regression. Sixty percent of the samples were selected randomly for using on the training and the remaining 30\% were used for testing, to evaluate the prediction. Finally, to predict the gait phase, the phasor variables were unpacked as

$$
\tilde{\varphi}[t]=\operatorname{atan} 2\left(\widetilde{\mathbb{Y}}_{2}[t], \widetilde{\mathbb{Y}}_{1}[t]\right) / 2 \pi
$$

where $\sim$ represents the estimated variable by the regression model and atan 2 is the arctangent function, returning an angle in the range $[0,2 \pi)$. Errors above $50 \%$ or below $-50 \%$ of the gait phase were added values of $-100 \%$ and $100 \%$, respectively, because the gait phase is a cyclic variable. For example, a gait phase error of $99 \%$ (above $50 \%)$ is equivalent to $-1 \%(99 \%-100 \%=$ $-1 \%)$.

\subsection{Experimental Procedure}

As a preliminary evaluation, three able-bodied subjects, including 2 males and 1 female, participated in a walking experiment in which their gait phase was predicted, and the environment was characterized. The subjects were able-bodied with no self-reported history of biomechanical or neuromuscular disorders. The walking course was an indoor level-ground hallway with $225 \mathrm{ft}$ in length (Figure 25). The subject walked along the perimeter of the hallway (at least 3 feet away from the walls) with a self-selected speed in clock-wise (CW) direction, then complete another lap in the opposite direction, in counter-clock-wise $(\mathrm{CCW})$ direction, totaling approximately $450 \mathrm{ft}$ of 
walking. The design of the track had 18 right-turns and 18 left-turns, and about resulted in approximately 100 steps in total. The subject was asked to change their gait speed throughout the course, but never stop walking, resulting in gait periods of $1.02 \pm 0.11 \mathrm{cycles} / \mathrm{s}$. In addition, the subject initially completed an extra lap so they would get used to the device, and the data from this lap was discarded.

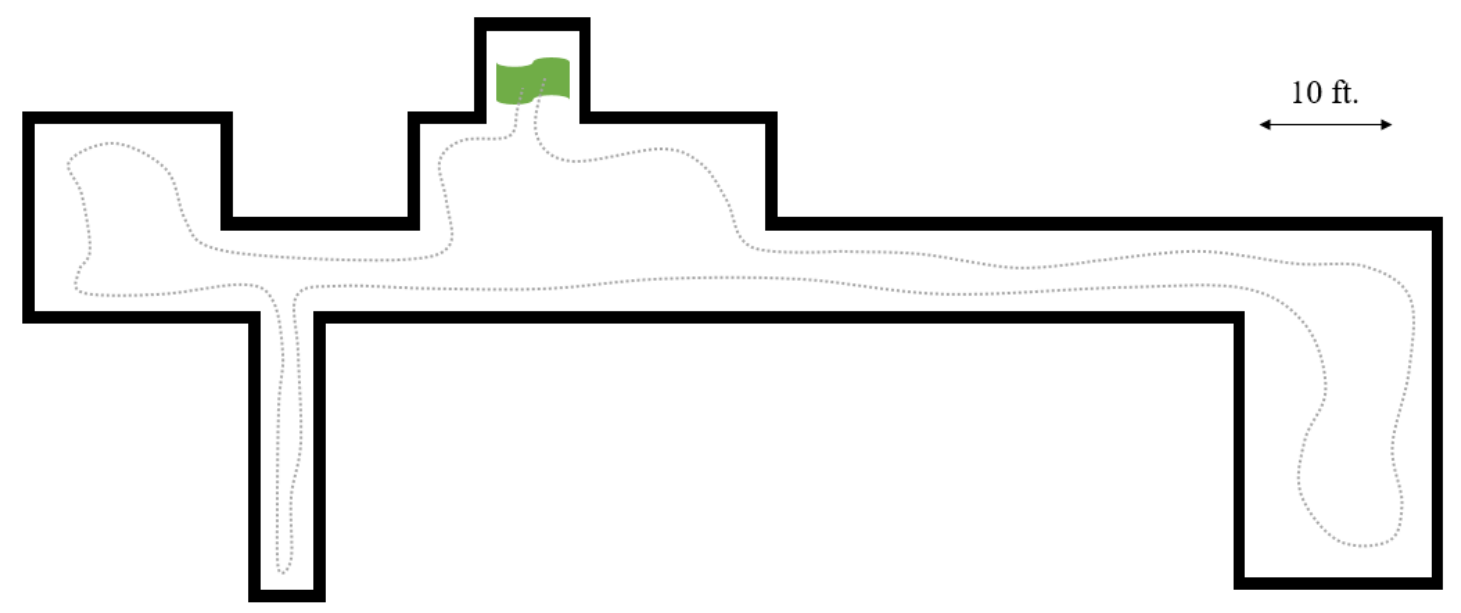

Figure 25. Walking course used for the evaluation of the VDGA. Subjects followed the perimeter of the hallway at a self-selected speed. The green flag indicates the start and end position.

The USB cables were attached to the outside of the upper shank and on the waist pack belt before connecting them to the onboard computer. This improved the mobility of the subject so that they could walk without interference from the cabling. The shank shell was taped to the shank behind the ankle (Figure 17.a) and on the upper shank (Figure 17.b) to avoid displacing the device during or between the experiments. The center of the FSR was placed approximately $30 \mathrm{~mm}$ from the posterior end of the shoe insole, centered along the mid-lateral direction. The subject wore their own personal, comfortable, shoes and socks.

\subsection{Results and Discussion}

The voltage measured on the FSR circuit ranged from $0 \mathrm{~V}$, when the FSR was unloaded, to approximately 3.2 V, when the FSR was under the subject's weight (Figure 26). For all subjects, the heel-strike event was detected when the voltage increased to above $2.0 \mathrm{~V}$. 
The voltage of the FSR stayed at $0 \mathrm{~V}$ during the later moments of the stance ( $40 \%$ to $60 \%$ ). This happened because the foot heel lost contact to the FSR, possibly because the shoe was unable to conform to the foot when the foot segments were deforming. However, during the swing phase, when the foot segments return to a neutral angle, the FSR restored the contact with the heel and increased the voltage. The drop of voltage was not used to label the gait phase because it depended on the flexibility of the subjects' shoes.

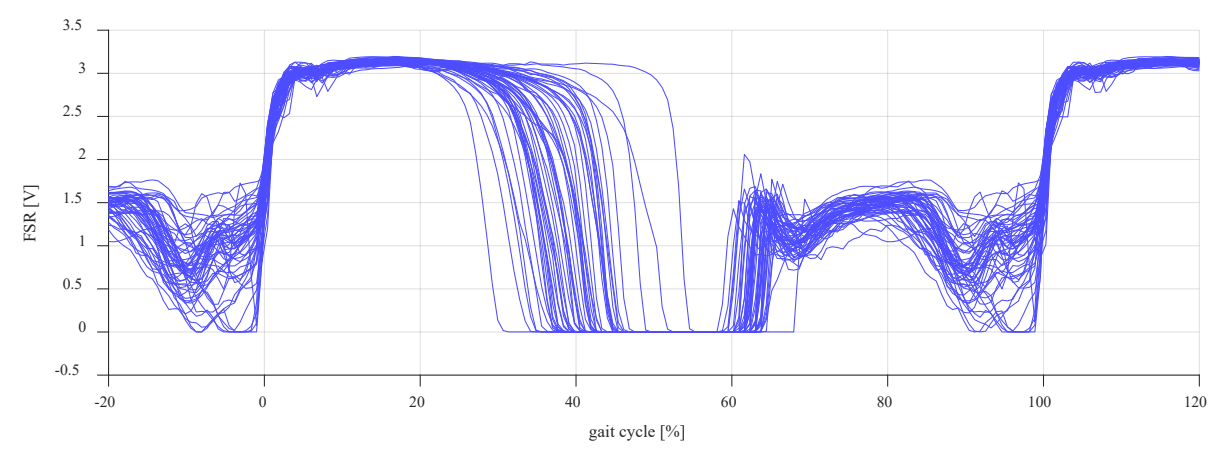

Figure 26. Voltage on the FSR circuit across the gait cycle for the subject I. The voltage was interpolated so that the heel strike happens at $0 \%$ and $100 \%$ marks.

The IMU measurements from multiple steps are shown in Figure 27 interpolated across the gait cycle. The small variance of the IMU readings indicates the FSR was able to segment the stance phase accurately. As expected, the IMU detected high accelerations close to the heel-strike and push-off events, and high angular acceleration along the sagittal plane (Y-axis) during the swing phase. 

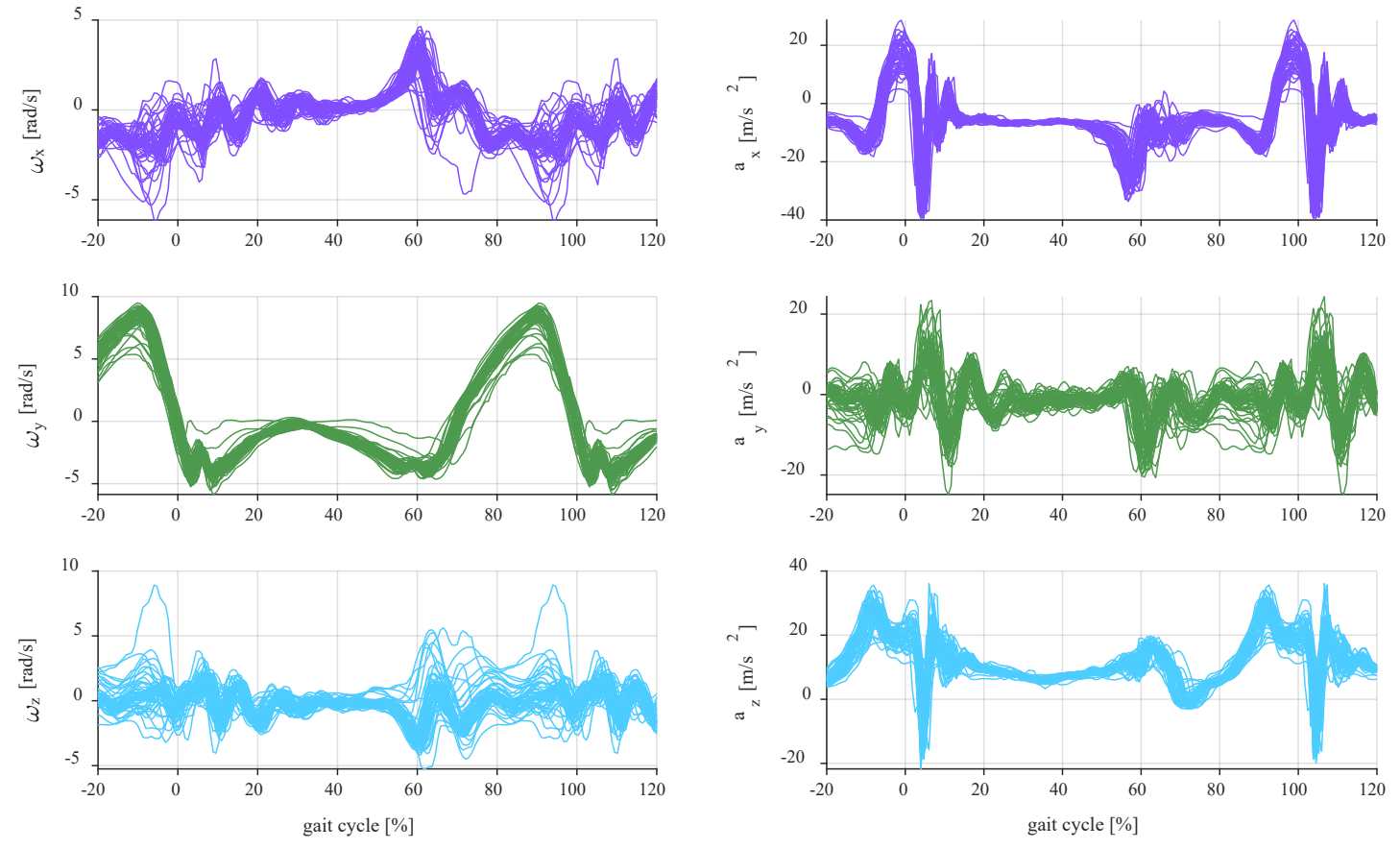

(a)

(b)

Figure 27. IMU measurements across the gait cycle for subject I. The (a) angular velocity and (b)

linear acceleration from the $\mathrm{X}, \mathrm{Y}$, and $\mathrm{Z}$ measurements, interpolated from multiple steps.

\subsubsection{Characterization of the Environment}

The normal vector of the ground predicted from the camera frame of a representative subject is shown in Figure 28. The normal vector was represented in the inertial frame; thus, it has a nominal value of $[0,0,1]^{T}$ (z-axis points up). Ideally, the normal vector estimate should not change across the gait phase or between different steps. The estimation was relatively accurate, except during the heel strike and push-off (gait phases of 0 and $60 \%$, respectively), in which the standard deviations of the plane model inclination along the $\mathrm{x}$ and $\mathrm{y}$ axes were about 4 degrees. The ground impact during heel strike greatly accelerates the camera, aggravating the effects on the plane estimation due to the camera rolling shutter (image pixels are not sampled at the same time) and synchronization errors between the IMU and the camera. The push-off gait event also showed relatively high ground reconstruction errors (standard deviation of errors around $3.5^{\circ}$ ). During the push-off, the camera's view was approximately perpendicular and at its closest distance to the ground, which reduced the ground area captured by the camera. With less ground area, but with the 
same amount of random noise (lower signal to noise ratio), the algorithm produced more errors estimating the plane model.
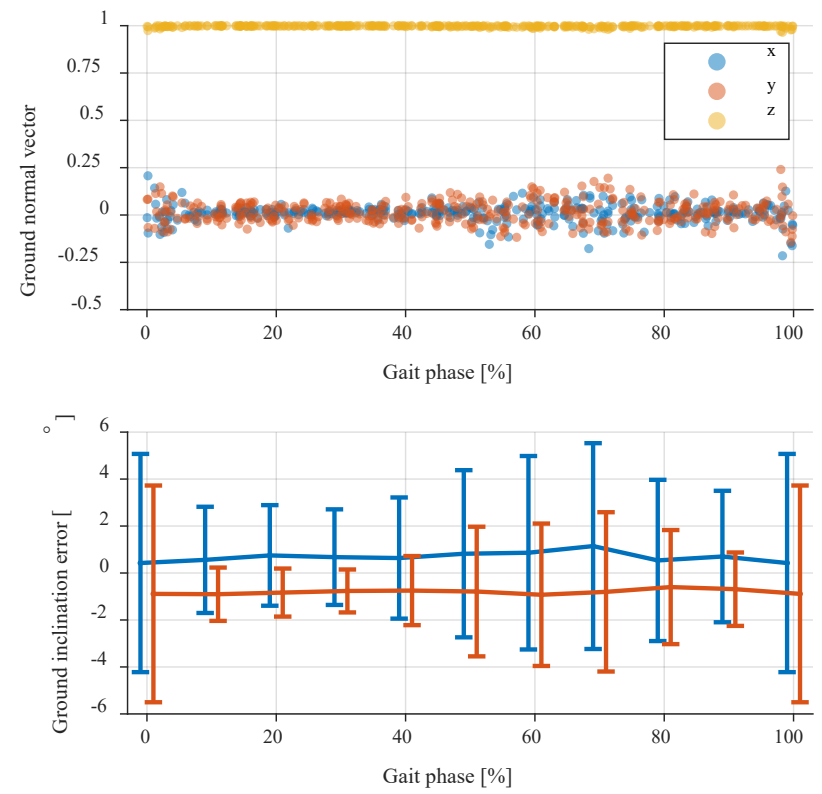

Figure 28. Normal vector estimates of the ground plane represented across the gait cycle. Each normal plane estimate, represented in the inertial frame, is shown on the top graph. The angle between each normal vector estimate and the average normal vector is considered as the estimation error, and is presented in the bottom graph.

The average ground inclination, represented in the inertial frame, was calculated for each trial (Table 4). Interestingly, there was a substantial non-zero ground inclination in every trial of about 3 degrees. However, this does not mean there was a real ground inclination in the walking course because each trial showed a different inclination estimate rather than the same. Also, an inclination of this magnitude would be noticeable (as a reference, the Pisa tower in Italy leaned about 4 degrees in 2013, and has leaned up to 10 degrees prior [76]). Thus, this error component likely arose from a bias noise from the IMU orientation, which uses a magnetometer and linear acceleration sensing. The standard deviation of the inclination was relatively similar for subjects and trials; and is likely due to 1) random measurement noise from the IMU and the camera, 2) small errors in the extrinsic calibration, and 3) small time-synchronization errors. 
Table 4. Environmental ground inclination for each experimental trial. The inclination error was calculated along the $\mathrm{X}$ and $\mathrm{Y}$ axes of the inertial frame.

\begin{tabular}{|c|c|c|c|}
\hline \multirow{2}{*}{ Subject } & \multirow{2}{*}{ Walking Track } & \multicolumn{2}{|c|}{ Ground Inclination } \\
\cline { 2 - 4 } & & $\mathbf{x}$-axis [ ${ }^{\circ}$ ] & y-axis [ $\left.{ }^{\circ}\right]$ \\
\hline \multirow{2}{*}{$I$} & $\mathrm{CW}$ & $0.67 \pm 3.28$ & $-0.84 \pm 2.54$ \\
\cline { 2 - 4 } & $\mathrm{CCW}$ & $2.14 \pm 3.00$ & $-0.17 \pm 2.67$ \\
\hline \multirow{2}{*}{$I I$} & $\mathrm{CW}$ & $-2.20 \pm 2.44$ & $2.09 \pm 2.34$ \\
\cline { 2 - 4 } & $\mathrm{CCW}$ & $-0.22 \pm 2.41$ & $-0.95 \pm 2.29$ \\
\hline \multirow{2}{*}{$I I I$} & $\mathrm{CW}$ & $-1.95 \pm 2.78$ & $0.20 \pm 2.81$ \\
\cline { 2 - 4 } & $\mathrm{CCW}$ & $0.95 \pm 2.55$ & $-2.93 \pm 2.33$ \\
\hline
\end{tabular}

The ground was characterized using instantaneous measurements of the IMU orientation and of the image depth. This analysis focused on evaluating the accuracy of the measurements and of the extrinsic calibration. However, a batch processing of many past measurements could result in an increased ground characterization. Such processing could use Simultaneous Localization and Mapping (SLAM) algorithms to estimate both the camera pose and the environment structure [77]. More recent SLAM algorithms also use depth and inertial measurements [78], [79] to increase the tracking stability of the camera pose and to capture the environment with more details. In addition, this system could improve stability and environment detection using prior knowledge of gait events measured by the FSR, similar to a dead-reckoning system for pedestrians [80]. This preliminary study shows promising results that indicate that the VDGA can be used for both gait prediction and environment characterization.

\subsubsection{Estimation of the Gait Cycle}

The histogram of the gait phase prediction errors for approach $a$ (IMU as predictor) and $b$ (IMU and depth as predictors) were calculated using the test samples (Figure 29). The gait phase prediction errors were $1.6 \pm 15.6 \%$ and $0.2 \pm 7.6 \%$ for approach $a$ and $b$, respectively. In other words, $49.9 \%$ and $90.8 \%$ of the predictions were within $[10 \%, 10 \%]$ of the correct value, for approach $a$ and $b$, respectively. For completeness, another set of features (angular velocity, linear acceleration, and orientation quaternion) was tested and also showed high prediction errors of 1.4 $\pm 15.6 \%$, similar to approach $a$. 


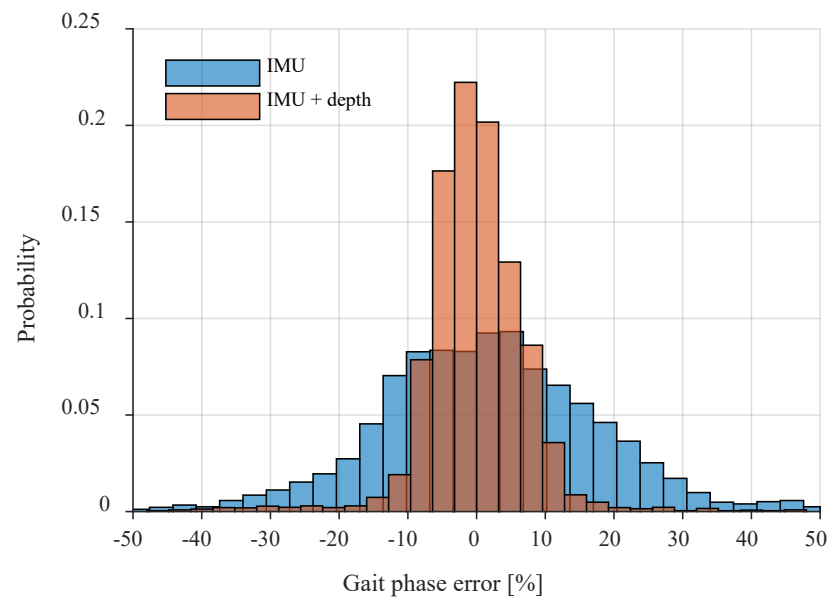

Figure 29. Histogram of the prediction error of the gait phase estimator. The approach using IMU and depth information have smaller errors compared to the IMU-only approach.

The visual information used on the second approach complemented the IMU measurements with the shank height and the shank orientation with respect to the ground. The IMU cannot estimate a linear positional variable directly (the double integration of linear acceleration would be imprecise), thus, an exteroceptive sensor such as the depth camera can add important information. In addition, it is likely the ground inclination could have a more substantial role for predicting the gait phase if the experiments were performed in more challenging ground environments, with slopes and steps, since similar phase predictors that use the thigh angular position and velocity must be compensated for the ground incline [81]. This preliminary study shows that the visual information can improve the prediction of the gait phase, and that combined with the estimates of the ground environment, might improve the operation of ankle-foot prosthesis in complex ground environments. 


\section{Conclusion}

In this work, the mechanical impedance of the human ankle was studied to improve the control of robotic ankle-foot prostheses. The mechanical impedance was shown to increase as a response to co-contractions of the calf muscles. The ankle stiffness around the DP anatomical axis was shown to increase up to four times when the calf muscles were at $40 \%$ of the MVC. The value of the ankle stiffness was also shown to depend on the mean angle and torque of a standing person, and to the direction of ankle rotation. As a consequence, a gait maneuver, which has varying levels of calf muscle activity, ankle angle, and torques, should present a varying impedance. To test this hypothesis, the multivariable and time-varying impedance of the ankle was estimated across the stance phase. Interestingly, the estimated stiffness and damping was approximately symmetric around the subtalar joint of the ankle, rather than around an anatomical axis.

The findings from the mechanical impedance experiments indicated the importance for robotic prostheses to recognize the state of the prosthesis user during the gait, as the user interacts with the environment. To further the work on robotic perception for ankle-foot prostheses, a wearable device called VDGA was designed and evaluated. This device is capable of characterizing the ground environment and estimating the gait state using a time-of-flight camera and an inertial measurement unit sensor. Preliminary tests estimated the level-ground environment with inclination errors of approximately three degrees, and predicted the gait phase variable with errors around $0.2 \pm 7.6 \%$ of the gait phase. These results showed that this device can capture accurate and relevant information for applications of robotic prostheses control.

As future work, the ankle impedance will be studied for different gait maneuvers and in complex environmental conditions, such as during ramp or stairs ascend or descend. In parallel, the VDGA algorithm will be extended to predict more features of the environment, such as stairs and ramps, and will be used to estimate the foothold of incoming steps. Finally, this work contributed to improve the prosthesis by identifying the mechanical behavior of the human ankle and by developing a platform to test perception algorithms for the control of robotic prostheses. 


\section{Reference List}

[1] K. Ziegler-Graham, E. J. MacKenzie, P. L. Ephraim, T. G. Travison, and R. Brookmeyer, "Estimating the prevalence of limb loss in the United States: 2005 to 2050," Arch. Phys. Med. Rehabil., vol. 89, no. 3, pp. 422-429, 2008.

[2] J. M. Robbins, G. Strauss, D. Aron, J. Long, J. Kuba, and Y. Kaplan, "Mortality Rates and Diabetic Foot Ulcers," J. Am. Podiatr. Med. Assoc., vol. 98, no. 6, pp. 489-493, Nov. 2008.

[3] "Elan - Carbon, Feet, Hydraulic, Sandal Toe," Endolite USA - Lower Limb Prosthetics. [Online]. Available: https://www.endolite.com/products/elan. [Accessed: 19-Jul-2019].

[4] “iWalk BiOM Foot / Ankle Prosthesis," MCOP Prosthetics. .

[5] "The technology behind the PROPRIO FOOT® from Össur." [Online]. Available: https://www.ossur.com/about-ossur/news-from-ossur/2-uncategorised/69-the-technologybehind-the-proprio-foot-from-ossur. [Accessed: 19-Jul-2019].

[6] N. M. Olson and G. K. Klute, "Design of a transtibial prosthesis with active transverse plane control," J. Med. Devices, vol. 9, no. 4, 2015.

[7] L. W. Lamoureux and C. W. Radcliffe, "Functional analysis of the UC-BL shank axial rotation device," Prosthet. Orthot. Int., vol. 1, no. 2, pp. 114-118, 1977.

[8] E. M. Ficanha and M. Rastgaar, "Preliminary design and evaluation of a multi-axis anklefoot prosthesis," in 5th IEEE RAS/EMBS International Conference on Biomedical Robotics and Biomechatronics, 2014, pp. 1033-1038.

[9] E. M. Ficanha and M. Rastgaar, "Impedance and admittance controller for a multi-axis powered ankle-foot prosthesis," in ASME 2014 Dynamic Systems and Control Conference, 2014.

[10] E. M. Ficanha, M. Rastgaar, and K. R. Kaufman, "A two-axis cable-driven ankle-foot mechanism," Robot. Biomim., vol. 1, no. 1, p. 17, 2014.

[11] E. M. Ficanha, M. Rastgaar, and K. R. Kaufman, "Control of a 2-DOF powered ankle-foot mechanism," in 2015 IEEE International Conference on Robotics and Automation (ICRA), 2015.

[12] E. M. Ficanha, G. A. Ribeiro, and M. Rastgaar, "Design and Evaluation of a 2-DOF Instrumented Platform for Estimation of the Ankle Mechanical Impedance in the Sagittal and Frontal Planes," IEEEASME Trans. Mechatron., vol. 21, no. 5, pp. 2531-2542, Oct. 2016.

[13] E. Ficanha, M. R. Aagaah, and K. R. Kaufman, "Cable-Driven Two Degrees-of-Freedom Ankle-Foot Prosthesis," J. Med. Devices, vol. 10, no. 3, 2016. 
[14] H. Dallali, E. Ficanha, and M. R. Aagaah, "Dynamic Modeling of a 2-DOF Cable Driven Powered Ankle-Foot Prosthesis," in ASME Dynamic Systems and Control Conference (DSCC), 2016.

[15] N. Dhir, H. Dallali, E. M. Ficanha, G. A. Ribeiro, and M. Rastgaar, "Locomotion envelopes for adaptive control of powered ankle prostheses," in 2018 IEEE International Conference on Robotics and Automation (ICRA), 2018, pp. 1488-1495.

[16] E. Ficanha, H. Dallali, and M. Rastgaar, "Gait Emulator for Evaluation of a Powered AnkleFoot Prosthesis," in ASME 2017 Dynamic Systems and Control Conference, 2017.

[17] E. Ficanha, M. Rastgaar, and K. R. Kaufman, "Gait Emulator for Evaluation of Ankle-Foot Prostheses Capable of Turning," J. Med. Devices, vol. 9, no. 3, 2015.

[18] P. L. Weiss, R. E. Kearney, and I. W. Hunter, "Position dependence of ankle joint dynamicsI. Passive mechanics," J. Biomech., vol. 19, no. 9, pp. 727-735, Jan. 1986.

[19] P. L. Weiss, R. E. Kearney, and I. W. Hunter, "Position dependence of ankle joint dynamicsII. Active mechanics," J. Biomech., vol. 19, no. 9, pp. 737-751, Jan. 1986.

[20] P. L. Weiss, I. W. Hunter, and R. E. Kearney, "Human ankle joint stiffness over the full range of muscle activation levels," J. Biomech., vol. 21, no. 7, pp. 539-544, 1988.

[21] E. S. Tehrani, K. Jalaleddini, and R. E. Kearney, "Ankle joint intrinsic dynamics is more complex than a mass-spring-damper model," IEEE Trans Neural Syst Rehabil Eng, vol. 25, no. 9 , pp. $1568-1580,2017$.

[22] E. Ficanha, G. Ribeiro, L. Knop, and M. Rastgaar, "Estimation of the Two Degrees-ofFreedom Time-Varying Impedance of the Human Ankle," J. Med. Devices, vol. 12, no. 1, 2018

[23] E. M. Ficanha, G. A. Ribeiro, L. Knop, and M. Rastgaar, "Time-varying impedance of the human ankle in the sagittal and frontal planes during straight walk and turning steps," in Rehabilitation Robotics (ICORR), 2017 International Conference on, 2017, pp. 1413-1418.

[24] E. J. Rouse, L. J. Hargrove, E. J. Perreault, and T. A. Kuiken, "Estimation of human ankle impedance during the stance phase of walking," IEEE Trans. Neural Syst. Rehabil. Eng., vol. 22, no. 4, pp. 870-878, 2014.

[25] H. Lee and N. Hogan, "Time-varying ankle mechanical impedance during human locomotion," IEEE Trans. Neural Syst. Rehabil. Eng., vol. 23, no. 5, pp. 755-764, 2015. 
[26] A. L. Shorter and E. J. Rouse, "Mechanical Impedance of the Ankle During the Terminal Stance Phase of Walking," IEEE Trans. Neural Syst. Rehabil. Eng., vol. 26, no. 1, pp. 135$143,2018$.

[27] E. M. Ficanha, M. Rastgaar, and K. R. Kaufman, "Ankle mechanics during sidestep cutting implicates need for 2-degrees of freedom powered ankle-foot prostheses.," J. Rehabil. Res. Dev., vol. 52, no. 1, 2015.

[28] M. S. Orendurff, A. D. Segal, J. S. Berge, K. C. Flick, D. Spanier, and G. K. Klute, "The kinematics and kinetics of turning: limb asymmetries associated with walking a circular path," Gait Posture, vol. 23, no. 1, pp. 106-111, 2006.

[29] E. M. Ficanha, R. Kang, and M. Rastgaar, "Ankle kinematics describing gait agility: Considerations in the design of an agile ankle-foot prosthesis," in 5th IEEE RAS/EMBS International Conference on Biomedical Robotics and Biomechatronics, 2014, pp. 132-137.

[30] E. M. Ficanha, M. Rastgaar, B. Moridian, and N. Mahmoudian, “Ankle angles during step turn and straight walk: Implications for the design of a steerable ankle-foot prosthetic robot," in ASME 2013 Dynamic Systems and Control Conference, 2013.

[31] H. Lee, P. Ho, M. Rastgaar, H. I. Krebs, and N. Hogan, "Multivariable static ankle mechanical impedance with active muscles," IEEE Trans. Neural Syst. Rehabil. Eng., vol. 22, no. 1, pp. 44-52, 2014.

[32] M. Rastgaar, H. Lee, E. Ficanha, P. Ho, H. I. Krebs, and N. Hogan, "Multi-directional dynamic mechanical impedance of the human ankle; A key to anthropomorphism in lower extremity assistive robots," in Neuro-Robotics, Springer, 2014, pp. 157-178.

[33] S. Rezazadeh, D. Quintero, N. Divekar, E. Reznick, L. Gray, and R. D. Gregg, "A phase variable approach for improved volitional and rhythmic control of a powered knee-ankle prosthesis," ArXiv Prepr. ArXiv181105414, 2018.

[34] S. Rezazadeh, D. Quintero, N. Divekar, and R. D. Gregg, "A phase variable approach to volitional control of powered knee-ankle prostheses," in 2018 IEEE/RSJ International Conference on Intelligent Robots and Systems (IROS), 2018, pp. 2292-2298.

[35] B. C. Glaister, G. C. Bernatz, G. K. Klute, and M. S. Orendurff, "Video task analysis of turning during activities of daily living," Gait Posture, vol. 25, no. 2, pp. 289-294, 2007.

[36] J. D. Ventura, A. D. Segal, G. K. Klute, and R. R. Neptune, "Compensatory mechanisms of transtibial amputees during circular turning," Gait Posture, vol. 34, no. 3, pp. 307-312, Jul. 2011. 
[37] H. Dallali, L. Knop, L. Castelino, E. Ficanha, and M. Rastgaar, "Estimating the multivariable human ankle impedance in dorsi-plantarflexion and inversion-eversion directions using EMG signals and artificial neural networks," Int. J. Intell. Robot. Appl., vol. 1, no. 1, pp. 19-31, 2017.

[38] H. Dallali, L. Knop, L. Castelino, E. Ficanha, and M. Rastgaar, "Using lower extremity muscle activity to obtain human ankle impedance in the external-internal direction," Int. $J$. Intell. Robot. Appl., vol. 2, no. 1, pp. 29-42, 2018.

[39] G. A. Ribeiro, L. N. Knop, and M. Rastgaar, "Correlation Between Ankle Impedance and EMG Signals," in International Conference on NeuroRehabilitation, 2018, pp. 627-631.

[40] L. N. Knop, G. A. Ribeiro, E. M. Ficanha, and M. Rastgaar, "Estimating the Relationship Between Multivariable Standing Ankle Impedance and Lower Extremity Muscle Activation," in 2018 7th IEEE International Conference on Biomedical Robotics and Biomechatronics (Biorob), 2018, pp. 285-290.

[41] L. Knop, G. A. Ribeiro, and M. Rastgaar, "Towards a Generalized Model of Multivariable Ankle Impedance During Standing Based on the Lower Extremity Muscle EMG," in 2019 Design of Medical Devices Conference, 2019.

[42] E. M. Ficanha and M. Rastgaar, "Stochastic estimation of human ankle mechanical impedance in lateral/medial rotation," in ASME 2014 Dynamic Systems and Control Conference, 2014.

[43] P. Ho, H. Lee, M. A. Rastgaar, H. I. Krebs, and N. Hogan, "Interpretation of the directional properties of voluntarily modulated human ankle mechanical impedance," in ASME 2010 Dynamic Systems and Control Conference, 2010, pp. 467-472.

[44] H. Lee, P. Ho, M. A. Rastgaar, H. I. Krebs, and N. Hogan, "Quantitative characterization of steady-state ankle impedance with muscle activation," ASME 2010 Dyn. Syst. Control Conf., pp. 321-323, 2010.

[45] A. S. McIntosh, K. T. Beatty, L. N. Dwan, and D. R. Vickers, "Gait dynamics on an inclined walkway,” J. Biomech., vol. 39, no. 13, pp. 2491-2502, Jan. 2006.

[46] A. S. Voloshina, A. D. Kuo, M. A. Daley, and D. P. Ferris, "Biomechanics and energetics of walking on uneven terrain,” J. Exp. Biol., vol. 216, no. 21, pp. 3963-3970, 2013.

[47] M. Liu, D. Wang, and H. H. Huang, "Development of an environment-aware locomotion mode recognition system for powered lower limb prostheses," IEEE Trans. Neural Syst. Rehabil. Eng., vol. 24, no. 4, pp. 434-443, 2016. 
[48] N. E. Krausz, T. Lenzi, and L. J. Hargrove, "Depth Sensing for Improved Control of Lower Limb Prostheses," IEEE Trans. Biomed. Eng., vol. 62, no. 11, pp. 2576-2587, Nov. 2015.

[49] Y. Massalin, M. Abdrakhmanova, and H. A. Varol, "User-Independent Intent Recognition for Lower Limb Prostheses Using Depth Sensing," IEEE Trans. Biomed. Eng., vol. 65, no. 8, pp. 1759-1770, Aug. 2018.

[50] B. H. Hu, N. E. Krausz, and L. J. Hargrove, "A Novel Method for Bilateral Gait Segmentation Using a Single Thigh-Mounted Depth Sensor and IMU," in 2018 7th IEEE International Conference on Biomedical Robotics and Biomechatronics (Biorob), Enschede, 2018, pp. $807-812$.

[51] Abraham. Savitzky and M. J. E. Golay, "Smoothing and Differentiation of Data by Simplified Least Squares Procedures.," Anal. Chem., vol. 36, no. 8, pp. 1627-1639, Jul. 1964.

[52] R. H. Byrd, J. C. Gilbert, and J. Nocedal, "A trust region method based on interior point techniques for nonlinear programming," Math. Program., vol. 89, no. 1, pp. 149-185, 2000.

[53] A. Ziai and C. Menon, "Comparison of regression models for estimation of isometric wrist joint torques using surface electromyography," J. Neuroengineering Rehabil., vol. 8, no. 1, p. 56, 2011.

[54] F. Zhang and H. Huang, "Source selection for real-time user intent recognition toward volitional control of artificial legs," IEEE J. Biomed. Health Inform., vol. 17, no. 5, pp. 907$914,2012$.

[55] K. Englehart and B. Hudgins, "A robust, real-time control scheme for multifunction myoelectric control," IEEE Trans. Biomed. Eng., vol. 50, no. 7, pp. 848-854, 2003.

[56] C. Castellini et al., "Proceedings of the first workshop on peripheral machine interfaces: Going beyond traditional surface electromyography," Front. Neurorobotics, vol. 8, p. 22, 2014.

[57] J. Wang, O. A. Kannape, and H. M. Herr, "Proportional EMG control of ankle plantar flexion in a powered transtibial prosthesis," in 2013 IEEE 13th International Conference on Rehabilitation Robotics (ICORR), 2013, pp. 1-5.

[58] S. Farmer, B. Silver-Thorn, P. Voglewede, and S. A. Beardsley, "Within-socket myoelectric prediction of continuous ankle kinematics for control of a powered transtibial prosthesis," $J$. Neural Eng., vol. 11, no. 5, 2014.

[59] H. Lee, H. I. Krebs, and N. Hogan, "Multivariable dynamic ankle mechanical impedance with active muscles," IEEE Trans. Neural Syst. Rehabil. Eng., vol. 22, no. 5, pp. 971-981, 2014. 
[60] H. Schaub and J. L. Junkins, Analytical mechanics of space systems. American Institute of Aeronautics and Astronautics, 2005.

[61] M. M. Mirbagheri, H. Barbeau, and R. E. Kearney, "Intrinsic and reflex contributions to human ankle stiffness: variation with activation level and position," Exp. Brain Res., vol. 135, no. 4, pp. 423-436, 2000.

[62] T. E. Sakanaka, M. Lakie, and R. F. Reynolds, "Sway-dependent changes in standing ankle stiffness caused by muscle thixotropy," J. Physiol., vol. 594, no. 3, pp. 781-793, 2016.

[63] P. Amiri and R. E. Kearney, "Ankle intrinsic stiffness changes with postural sway," J. Biomech., vol. 85, pp. 50-58, Mar. 2019.

[64] H. Huang, T. A. Kuiken, and R. D. Lipschutz, "A strategy for identifying locomotion modes using surface electromyography," IEEE Trans. Biomed. Eng., vol. 56, no. 1, pp. 65-73, 2008.

[65] R. F. Chandler, C. E. Clauser, J. T. McConville, H. M. Reynolds, and J. W. Young, "Investigation of inertial properties of the human body," Air Force Aerospace Medical Research Lab Wright-Patterson AFB OH, 1975.

[66] P. L. Weiss, R. E. Kearney, and I. W. Hunter, "Position dependence of ankle joint dynamicsII. Active mechanics," J. Biomech., vol. 19, no. 9, pp. 737-751, 1986.

[67] E. K. Antonsson and R. W. Mann, “The frequency content of gait," J. Biomech., vol. 18, no. 1, pp. 39-47, 1985.

[68] H. Lee, E. J. Rouse, and H. I. Krebs, "Summary of human ankle mechanical impedance during walking," IEEE J. Transl. Eng. Health Med., vol. 4, pp. 1-7, 2016.

[69] ROS driver for the pmd CamBoard pico flexx. Contribute to code-iai/pico_flexx_driver development by creating an account on GitHub. Institute for Artificial Intelligence University of Bremen, 2019.

[70] Unified sensor driver for the Adafruit BNO055 orientation sensor breakout: adafruit/Adafruit_BNO055. Adafruit Industries, 2019.

[71] B. Sensortec, "BNO055 Intelligent 9-axis absolute orientation sensor," Bosch Sensortec Baden-Württ. Ger., 2016.

[72] P. Furgale, J. Rehder, and R. Siegwart, "Unified temporal and spatial calibration for multisensor systems," in Intelligent Robots and Systems (IROS), 2013 IEEE/RSJ International Conference on, 2013, pp. 1280-1286. 
[73] J. Rehder, J. Nikolic, T. Schneider, T. Hinzmann, and R. Siegwart, "Extending kalibr: Calibrating the extrinsics of multiple IMUs and of individual axes," in 2016 IEEE International Conference on Robotics and Automation (ICRA), 2016, pp. 4304-4311.

[74] M. A. Fischler and R. C. Bolles, "Random sample consensus: a paradigm for model fitting with applications to image analysis and automated cartography," Commun. ACM, vol. 24, no. 6, pp. 381-395, 1981.

[75] R. B. Rusu and S. Cousins, "Point cloud library (pcl)," in 2011 IEEE international conference on robotics and automation, 2011, pp. 1-4.

[76] "Napkin Math: Will the Leaning Tower of Pisa Ever Fall?," National Geographic, 02-Nov2013. [Online]. Available: https://www.nationalgeographic.com/people-andculture/onward/2013/11/02/napkin-math-will-the-leaning-tower-of-pisa-ever-fall/. [Accessed: 28-Jul-2019].

[77] M. Montemerlo, S. Thrun, D. Koller, and B. Wegbreit, "FastSLAM: A factored solution to the simultaneous localization and mapping problem," Aaai/iaai, pp. 593-598, 2002.

[78] T. Laidlow, M. Bloesch, W. Li, and S. Leutenegger, "Dense RGB-D-inertial SLAM with map deformations," in 2017 IEEE/RSJ International Conference on Intelligent Robots and Systems (IROS), 2017, pp. 6741-6748.

[79] R. Mur-Artal and J. D. Tardós, “Orb-slam2: An open-source slam system for monocular, stereo, and rgb-d cameras," IEEE Trans. Robot., vol. 33, no. 5, pp. 1255-1262, 2017.

[80] H. Ju, M. S. Lee, S. Y. Park, J. W. Song, and C. G. Park, “A pedestrian dead-reckoning system that considers the heel-strike and toe-off phases when using a foot-mounted IMU,"Meas. Sci. Technol., vol. 27, no. 1, 2015.

[81] D. Quintero, D. J. Villarreal, D. J. Lambert, S. Kapp, and R. D. Gregg, "Continuous-phase control of a powered knee-ankle prosthesis: Amputee experiments across speeds and inclines," IEEE Trans. Robot., vol. 34, no. 3, pp. 686-701, 2018. 